(C) October, 2007

Tianjing Hu

All Rights Reserved 


\title{
REGULATION OF PROCESS RETRACTION AND CELL MIGRATION BY EPHA3 IS MEDIATED BY THE ADAPTOR PROTEIN NCK1
}

\author{
By \\ TIANJING HU \\ A Dissertation submitted to the \\ Graduate School of New Brunswick \\ Rutgers, The State University of New Jersey \\ and \\ The Graduate School of Biomedical Sciences \\ University of Medicine and Dentistry of New Jersey \\ in partial fulfillment of the requirements \\ for the degree of \\ Doctor of Philosophy \\ Graduate Program in Neuroscience \\ Written under the direction of \\ Dr. Renping Zhou, Ph. D. \\ and approved by
}

New Brunswick, New Jersey

October, 2007 


\section{ABSTRACT OF THE DISSERTATION \\ REGULATION OF PROCESS RETRACTION AND CELL MIGRATION BY EPHA3 IS MEDIATED BY THE ADAPTOR PROTEIN NCK1}

By TIANJING HU

\section{Dissertation director: \\ Dr. Renping Zhou}

Members of the largest RTK (receptor tyrosine kinase) family, Eph receptors, and their ligands play pivotal roles in embryonic development. They have been shown to regulate axon guidance, cell migration, tissue boundary formation, synaptogenesis, and angiogenesis. In addition, mis-regulation of the Eph receptor function leads to tumorigenesis and possibly other diseases. However, little is known about signaling pathways downstream of Eph receptors. To shed light on signaling mechanisms of Eph receptors, we conducted a large-scale yeast two-hybrid screen to identify protein that interact with EphA receptors. In this screen, many promising candidates were identified, several of which warrant future investigation. A SH2/SH3 adaptor protein, Nck1, stands out as the strongest interactor with both EphA3 and EphA5. To better understand the signaling mechanisms of the EphAs and the role of Nck1, we performed in-depth studies on fibroblast cells and primary neurons. Here we present evidence that Nck1 interacts directly with activated EphA3 receptor through its $\mathrm{SH} 2$ domain and the juxtamembrane domain tyrosine residue 602 on EphA3. We find that the disruption of Nck1 binding by either SH2 domain or SH3 domains negatively regulates EphA3-mediated signaling 
during cell migration and cell process extension in HEK293A cells. Intriguingly, we also find that expression of Nck1 mutants through adenoviruses is not able to prevent growth cone collapse in primary hippocampal explants. These observations suggest that Nck1 plays a key role in mediating some aspects of EphA receptor functions.

Key words: Eph, ephrin, Receptor tyrosine kinase, SH2 domain, Nck1, Yeast twohybrid, Hippocampal neuron culture. 


\section{DEDICATIONS}

To my wonderful parents, Mr. Ming Hu and Ms. Cuiying Liao

I am forever in debt to you for your love, trust and support.

Without your encouragements,

I would not have been able to travel half across the globe,

and experience a completely different life.

You set great examples for me,

by working hard and treating others with sincerity, respect and kindness.

Your wisdom will be an endless treasure, from which I draw great strengths during my entire life. 


\section{ACKNOWLEDGEMENTS}

I am tremendously indebted to my advisor Dr. Renping Zhou, for his guidance and support during my graduate studies. His insights on many subjects influence me deeply.

Many thanks go to everyone in the lab of Dr. Zhou. I especially thank Dr. Guanfang Shi for her selfless help and technical expertise. I would also like to extend my gratitude towards all people in Dept. of Neuroscience and Lab for Cancer Research, who worked with me and gave me precious advice, especially Dr. James Zheng, Dr. Shu-Chan Hsu, Dr. David Egger, Dr. George Wagner, Dr. Allan Conney, Dr. Susie Chen, Dr. Nan-joo Suh, Dr. Audrey Minden and Dr. Lisa Epstein Wong.

Last but not least, I want to thank all the friends I have made during the time in Rutgers. Without their company, my life in Rutgers would not have been so memorable. Among them, Ms. Emily Su, Ms. Maggie Cooper, Mr. Kam Jim, Mrs. Cindy Lewicki, and Dr. Fei Xu are my closest friends. We have grown together and their friendship helps me become the person I am today. 


\section{TABLE OF CONTENTS}

$\begin{array}{ll}\text { Abstract } & \text { ii }\end{array}$

Dedications $\quad$ iv

Acknowledgements $\quad$ v

Table of contents $\quad$ vi

List of tables $\quad$ xi

List of illustrations $\quad$ xii

Chapter I. Background

Part I. The Eph receptors and their ligands 3

1.1.1. Receptor tyrosine kinases 3

1.1.2 Molecular properties of Eph receptors and ephrins 5

$\begin{array}{ll}\text { 1.1.3 Activation of Eph receptors } & 7\end{array}$

1.1.4 Signal transduction after Eph activation 9

$\begin{array}{lll}\text { 1.1.5 Adhesion vs. repulsion } & 10\end{array}$

1.1.6 Eph/ephrin signaling is unique among RTKs 13

Part II. Functions of Eph and ephrin signaling 14

$\begin{array}{lll}\text { 1.2.1 Axon guidance } & 14\end{array}$

$\begin{array}{ll}\text { 1.2.2 Axon branching } & 17\end{array}$

$\begin{array}{lll}\text { 1.2.3 Axon fasciculation and defasciculation } & 18\end{array}$

1.2.4 Spine formation $\quad 18$

1.2.5 Cell proliferation and differentiation 20

1.2.6 Tissue patterning 20 
$\begin{array}{lll}\text { 1.2.7 Cell migration } & 21\end{array}$

$\begin{array}{lll}\text { 1.2.8 Angiogenesis } & 22\end{array}$

$\begin{array}{lll}\text { 1.2.9 } & \text { Tumorigenesis } & 22\end{array}$

Part III. Signaling pathways downstream of activated Eph receptors and ephrins. 24

1.3.1 Binding proteins of Eph receptors 24

1.3.2 Eph signaling: Src family kinases and Nck1 25

1.3.3 Eph signaling: Ras/MEK/ERK pathway 28

1.3.4 Eph signaling: $\mathrm{PIP}_{2}$ related pathways 30

1.3.5 Eph signaling: small GTPases 31

1.3.6 Eph signaling: cell adhesion 33

1.3.7 Reverse signaling through ephrin-Bs 35

1.3.8 Reverse signaling through ephrin-As 38

$\begin{array}{ll}\text { Summary } & 39\end{array}$

Chapter II. Screening for EphA receptor binding proteins using the Yeast two-

$\begin{array}{ll}\text { hybrid method } & 58\end{array}$

2. 1. Introduction $\quad 58$

2. 2. Results

2.2.1 Construction of the bait plasmid and screening of a mouse brain cDNA

$\begin{array}{lr}\text { library } & 59\end{array}$

2.2.2 Fifteen interactors were identified through the screen 61

2.2.3 Nck1 binds strongly with EphA5 receptor 62

$\begin{array}{ll}\text { 2. 3. Discussion } & 63\end{array}$ 
2.3.1 The identified EphA interactors regulate different aspects of signal transduction

2.3.2 The binding of Nck1 and EphA receptors links activated EphA receptor and the cytoskeleton regulation.

Chapter III. Characterization of EphA3-Nck1 binding and evaluation of Nck1 in

EphA3 signaling

3. 1. Introduction

3. 2. Results

3.2.1. Interaction between Nck1 and EphA3 is mediated by the binding of Nck1 SH2 domain to the tyrosine 602 of EphA3

3.2.2 EphA3 and Nck1 form a protein complex in ephrin-A5-stimulated cells 85

3.2.3 Nck1 mutants down-modulate ephrin-A5-mediated inhibition of cell migration

3.2.4 Nck1 mutants inhibit ephrin-A5-induced cell process retraction

3.3.1 Nck1 interacts with activated EphA3

3.3.2 Nck1 mediates regulation of cell migration and process retraction by EphA3 activation

Chapter IV. Analysis of EphA3-Nck1 binding in neurons and assessment of Nck1 in hippocampal neuron growth cone collapse

116

4. 1. Introduction 116

4. 2. Results 
4.2.2 EphA3 and Nck1 interact in hippocampal neurons and cortical neurons

4.2.3 Ephrin-A5 induces growth cone collapse in a hippocampal neuron culture

4.2.4 Construction of adenoviruses carrying wild type and dominant negative clones of Nck1

4.2.5 Nck1 DN mutants do not prevent growth cone collapse induced by ephrin-A5

4. 3. Discussion

4.3.1 Nck1 binds to activated EphA3 receptor in neurons and they co-localize highly on the growth cones

4.3.2 Nck1 signaling may regulate aspects of EphA3 signaling but not growth cone collapse

Chapter V. Assessment of the binding of EphA3 with Grb2 and RasGAP

5.2.1 The SH2 domain of both Grb2 and RasGAP bind strongly to EphA3 143

5.2.2 Tyrosine 602 on EphA3 is a binding site for Grb2 SH2 domain

5.2.3 RasGAP binds to EphA3 partially through tyrosine 602 145

\section{3. Discussion}

5.3.1 EphA3 interacts with multiple $\mathrm{SH} 2$ domain containing proteins

5.3.2 Grb2 may compete with Nck1 for the binding of EphA3

5.3.3 RasGAP binds to EphA3 through multiples sites including tyrosine 602148

5.3.4 Other $\mathrm{SH} 2$ domain proteins that interact with the juxtamembrane domain 149 
Chapter VI. Materials and Methods

Chapter VII. Conclusions

7. 1. Novel binding proteins are implicated in $\operatorname{Eph} A$ receptor signaling

7. 2. Nck1, Grb2 and RasGAP all bind to tyrosine 602 through their SH2

domains

7. 3. Nck1 is an integral part of EphA3 mediated signaling

References

176

Curriculum Vitae

198 


\section{LIST OF TABLES}

Table 1-1. Signaling proteins that interact with Eph receptors.

Table 1-2. Signaling proteins downstream of ephrin ligands.

Table 2-1. A summary of fifteen identified binding proteins of EphA5 in the yeast two-hybrid screen. 


\section{LIST OF ILLUSTRATIONS}

Figure 1-1. Domain structure of Eph receptors and ephrin ligands.

Figure 1-2. Phylogram of Eph family kinases and class-specific interactions

between Eph receptors and ephrins.

Figure 1-3. Eph/ephrin activation, proteolysis, and endocytosis.

Figure 1-4. A schematic representation of the retinal-tectal projection in chick. 47

Figure 1-5. Signaling pathways implicated in Eph receptor mediated signal

52 transduction.

Figure 1-6. Signaling pathways involved in reverse signaling through ephrin-Bs. 56

Figure 2-1. A schematic representation of the yeast two-hybrid screen.

Figure 2-2. A summary of the yeast two-hybrid screen procedure.

69

Figure 2-3. Structure and expression of the bait LexA-EphA5intra.

Figure 2-4. Large-scale preliminary screens for EphA5 bait through LacZ reaction.

Figure 2-5. Yeast mating test of the binding proteins identified in earlier screens. 75

Figure 2-6. Nck1, a strong binding protein of EphA5.

Figure 3-1. Interaction of Nck1 with EphA3 through SH2 domain. 96

Figure 3-2. A series of EphA3 mutants containing single tyrosine mutations. $\quad 98$

Figure 3-3. Identification of the Tyr602 as theNck1 binding tyrosine residue on

EphA3.

Figure 3-4. Precipitation of EphA3 by Nck1 antibody in 293A cells.

Figure 3-5. Co-localization of Nck1 and EphA3 in transfected HEK 293A cells. 104

Figure 3-6. EphA3-induced inhibition of cell migration mediated by Nck1.

106 
Figure 3-7. Cell process retraction induced by activation of EphA3.

Figure 3-8. Disruption of EphA3-induced process retraction by Nck1 DN genes. 110 Figure 3-9. Quantification of Nck1 mutant effects on EphA3-induced process retraction.

Figure 3-10. A model of the effects of Nck1 DN genes in EphA3 signaling.

Figure 4-1. Expression of EphA3 and Nck1 in cortical and hippocampal neuron cultures.

Figure 4-2. Co-localization of EphA3 and Nck1 in hippocampal neuron growth cones.

Figure 4-3. Co-precipitation of EphA3 and Nck1 in cortical neurons.

Figure 4-4. Growth cone collapse induced by ephrin-A5 treatment in hippocampal neurons.

Figure 4-5. A schematic outline of the construction of adenoviral vectors carrying the gene of interest.

Figure 4-6. Growth cone collapse assay on neurons infected with adenoviruses expressing Nck1WT, Nck1R308K, and Nck1W38/143/229K.

Figure 5-1. Interaction of EphA3 with Grb2 SH2 domain and RasGAP N-terminal SH2 domain.

Figure 5-2. Identification of Tyr602 as one binding site of Grb2 protein.

Figure 5-3. Identification of Tyr602 as one of RasGAP binding sites on EphA3. 155 Figure 5-4. Domain structure of all SH2 domain containing proteins that bind to Eph receptors. 
Figure 5-5. A schematic representation of the interactions of EphA3 receptor with

Grb2 and RasGAP.

Figure 7-1. A summary of EphA3 signal transduction pathway involving Nck1, Grb2 and RasGAP. 


\section{Chapter I. Background}

The first member of Eph receptors was identified in 1987 in hepatocellular carcinoma cells (Hirai et al. 1987). Up to this date, a total of 16 members have been discovered. They constitute the largest Receptor Tyrosine Kinase (RTK) family. The ligands for Eph receptors are called "ephrins". A sum of 8 ephrins have been isolated so far in mammals (Beckmann et al. 1994; Davis et al. 1994). Eph and ephrin family members are expressed highly in a wide variety of tissues in both developing and adult organisms (Nakamoto 2000; Boyd and Lackmann 2001). Consistent with their wide expression, Eph receptors and ephrins are implicated in diverse biological processes, including but not limited to: axon projection, axon fasciculation, cell migration, cell sorting, tissue patterning, synaptogenesis, neuronal spine modulation, angiogenesis, and tumorigenesis (Boyd and Lackmann 2001; Wilkinson 2001; Kullander and Klein 2002; Pasquale 2005; Heroult et al. 2006).

Much progress on elucidating the mechanisms of Eph receptor signaling has been made in recent years. These signaling pathways become activated downstream of Eph receptors and regulate Ras-like small GTPase activity, actin cytoskeleton, integrin signaling, MAPK activity and more (Lawrenson et al. 2002; Huot 2004; Meyer et al. 2005; Sahin et al. 2005). However, detailed mechanisms of these pathways remain unclear. Thus, a study was undertaken to gain insights into the signal transduction mechanisms of Eph receptors.

We began our study with a large-scale screen for EphA interacting proteins. The screen was conducted with the intracellular domain of EphA5 (EphA5intra) as the bait 
against a mouse embryonic brain cDNA library, using the DupLEX yeast two-hybrid system. Through this screen, fifteen novel binding proteins were identified and confirmed later by yeast mating tests. Among these positives, Nck1, a SH2/SH3 domain protein, displays the strongest interaction with EphA5. Interestingly, Nck1 was also identified when a similar screen using EphA3 bait was performed. Previously, Nck1 has been shown to play critical roles in signaling events downstream of other RTK family members, including EphB receptors (Holland et al. 1997; Tang et al. 1997; Stein et al. 1998a; Rivera et al. 2006). However, its interaction with EphA receptors has never been established. The interaction of Nck1 and EphA3 therefore became the focus of the current study.

In our study, we confirmed the direct interaction of Nck1 and EphA3. The interaction sites on both proteins were identified, using GST fusion proteins and a group of EphA3 mutants. Nck1 dominant negative mutants were used to show Nck1 activity was necessary in EphA3 signaling in 293A cells. Furthermore, the expression of Nck1 in primary neuron culture was determined and its role in EphA3 mediated growth cone collapse assessed. Additionally, the binding of two other SH2 domain proteins Grb2 and RasGAP with EphA3 was evaluated.

Chapter I provides the background to the current study and consists of (1) a general introduction to the characteristics of Eph receptors and their ligands. (2) a discussion of various functions regulated by Eph and ephrin signaling. (3) a summary of signaling proteins for Eph receptors as well as ephrins and the signaling pathways they regulate. 


\section{Part I. The Eph receptors and their ligands}

\section{Receptor tyrosine kinases}

Eph receptors belong to the Receptor Tyrosine Kinase (RTK) superfamily. RTKs are transmembrane receptors that have intrinsic kinase activity. To date, fifty-eight receptor tyrosine kinases have been found through a genome-wide search in human (Pawson 1995; Robinson et al. 2000). Based on sequence homology, these receptors are further categorized into 19 classes (Shurin et al. 1998; Mellitzer et al. 2000). Well-known examples of the RTK superfamily include epidermal growth factor receptors (EGFR, ErbB), insulin receptors (INSR), platelet-derived growth factor receptors (PDGFR), hepatocyte growth factor receptors (HGFR) and nerve growth factor receptors (NGFR or TRK).

In general, RTKs are composed of three parts, an extracellular ligand binding domain, a transmembrane domain, and an intracellular kinase domain. The extracellular domain is usually composed of multiple domain modules such as Immunoglobin like domains, Fibronectin type III domains, Cysteine-rich repeats and Leucine-rich regions (Zwick et al. 2001). For example, TrkA, a receptor for NGF, consists of one Cysteinerich domain and two Immunoglobin like domains on the extracellular fragment (Holden et al. 1997).

The ligands of RTKs are usually secreted and they reach target cells through diffusion (Zwick et al. 2002; Barbieri et al. 2004). However, some RTKs such as Eph receptors, bind to membrane-bound ligands exclusively. Binding of ligands and receptors within a class is usually promiscuous: that is, ligands of one RTK class may bind to 
multiple receptors in this class and vice versa (Zwick et al. 2001). The specificity of the receptor/ligand binding is largely dependent on the conformation of the extracellular ligand-binding domain of the RTKs.

Unstimulated receptor tyrosine kinases are un-clustered and inactive. Once the diffusible ligands reach the cell surface and engage the ligand-binding sites on the RTKs, the receptor molecules undergo immediate dimerization. Although most dimerizations occur between two identical receptors, hetero-dimerization occurs as well. (Zwick et al. 2001; Normanno et al. 2006). For example, two isoforms of PDGF receptors exist and they are capable of dimerizing with each other in response to PDGF activation (Eriksson et al. 1992).

Following receptor dimerization, conformational changes on the intracellular domain of the receptors take place (Zwick et al. 2001; Barbieri et al. 2004; Zhang and Hughes 2006). These changes are critical to RTK function because they directly lead to the activation of kinase activity on the receptors. The kinase active receptors transphosphorylate each other on key tyrosine residues. The addition of negatively charged phosphate groups to the intracellular segment further enhances the kinase activity. More importantly, the tyrosine phosphorylation on the receptors provides important docking sites for effector proteins.

Some signaling proteins serve as scaffolds and enable effector proteins to associate with the activated RTKs. Since some effectors are directly phosphorylated by RTKs, association of these effectors with RTKs activates them. Consequently, the activated effectors transduce signals from membrane bound receptors to the signaling cascade including the MAPK, PI3 kinase, PLC gamma and JNK pathways (Zwick et al. 2001; 
Oda et al. 2005). Because they are capable of triggering the activation of diverse signaling pathways, RTKs play a pivotal role in the regulation of cellular functions such as cell survival, proliferation, differentiation, adhesion, and apoptosis. Moreover, the many functions of RTK, implicate them in the progression of diseases such as cancer (Avruch et al. 2001). Indeed, one of the focal points of RTK research now involves the application of RTK receptor inhibitors to cancer treatment (Shinkaruk et al. 2003; Carlomagno and Santoro 2005).

RTK superfamily members share many common characteristics, including the activation process and the downstream pathways. The understanding of the mechanisms of other RTK members helps to shed light on Eph signaling.

\section{Molecular properties of Eph receptors and ephrins}

Eph receptors were named after "erythropoietin producing human hepatocellular cell line", from which the first Eph receptor was isolated (Hirai et al. 1987). The Eph family includes a total of 16 members in mammals, which comprise the largest class in the RTK superfamily (Flanagan and Vanderhaeghen 1998; Himanen and Nikolov 2003a; Sasaki et al. 2003; Aasheim et al. 2005). Eph family members have highly conserved structures. Starting from the N-terminus, they consist of a globular ligand binding domain, a Cysteine-rich domain, two Fibronectin type III motifs, a juxtamembrane domain, a tyrosine kinase domain, a SAM domain and a PDZ binding motif (Fig. 1-1 A). Analysis of the DNA sequence of Eph receptors reveals that they share a high degree of homology, especially in the kinase domain (Fig. 1-2 A). 
The ligands for Ephs are named "ephrins", the abbreviation of "Eph receptor interacting proteins". Being membrane-bound through a GPI anchor or a transmembrane domain, they are also highly conserved (Nakamoto 2000; Himanen and Nikolov 2003b). Depending on the type of connection to the cell membrane, ephrins are divided into two subclasses: the A subclass, which are anchored through a GPI link and lack a cytoplasmic fragment, and the B subclass, which have a transmembrane domain and a cytoplasmic tail (Fig. 1-1 B, C). In mammals, five ephrin-As and three ephrin-Bs have been identified so far (Frisen et al. 1999; Blits-Huizinga et al. 2004).

Based on sequence homology and ligand binding, Eph receptors are also categorized into A and B subclasses. In general, one receptor has higher sequence homology with other receptors of the same subclass than with receptors of a different subclass (Zhou 1998). Moreover, receptors of one subclass interact only with the ligands in the corresponding ligand subclass; although, within each subclass, the ligands and receptors bind promiscuously (Gale et al. 1996b). There exist some exceptions to the subclass specificity of receptor-ligand binding (Fig. 1-2 B). For example, EphA4 interacts with both the A- and the B-ephrins while EphB2 also binds ephrin-A5 in addition to the Bephrins (Gale et al. 1996b; Himanen et al. 2004; Pasquale 2004). It has been reported that ephrin-A1, -A3 and -A4 bind to EphB1 with low affinity (Beckmann et al. 1994); however, it is unclear whether these interactions lead to any significant biological functions.

Site mutagenesis and crystal structure studies reveal that the "H-I" loop, located on the ephrin binding domain on Eph receptors, is critical in determining the subclass specificity of Eph/ephrin interaction (Himanen and Nikolov 2003b; Pasquale 2004). The 
$\mathrm{H}-\mathrm{I}$ loop is a part of the "low affinity receptor-ligand binding interface". Its length is conserved within each subclass, but different between the two subclasses. The significance of this loop was further supported by the preferential binding to A class ligands by EphB2 when its H-I loop was replaced by the H-I loop of Eph A3. (Himanen et al. 1998). Consequently, the H-I loop is termed the "class-specificity loop".

\section{Activation of Eph receptors}

Eph receptors and ephrins are expressed at high levels in a wide variety of tissues such as kidney, lung, heart, and muscle. The highest expression, however, is found in the nervous system, especially during development (Zhou 1998). Usually, Eph receptors and ephrins are found on different populations of cells and the receptor-ligand interaction produces repulsive effects, which lead to repulsive effects such as tissue boundary formation or axon retraction (Flanagan and Vanderhaeghen 1998; Knoll and Drescher 2002). Attractive effects are also observed in some biological events (Magal et al. 1996; Gao et al. 2000; Zhou et al. 2001; Hindges et al. 2002a; Holmberg and Frisen 2002; Eberhart et al. 2004). In addition to their roles during development, Eph receptors have been shown to regulate adult functions such as learning and memory (Dalva et al. 2000; Grunwald et al. 2001b; Henderson et al. 2001; Takasu et al. 2002b), and pathological conditions such as tumorigenesis (Brantley-Sieders et al. 2004; Surawska et al. 2004). These functions will be discussed in more detail in Part II.

Before Eph/ephrin signaling starts, ephrin molecules form loosely bound dimers (Himanen et al. 2001). By contrast, Eph receptors usually remain monomers (Fig. 1-3 A). 
On the unstimulated Eph monomers, the kinase activity is inhibited because the kinase domain is blocked by the juxtamembrane domain (Wybenga-Groot et al. 2001).

When Eph expressing cells and ephrin expressing cells encounter each other, the Eph monomers bind to ephrin dimers on the opposing cells through a "high affinity receptor-ligand binding interface". The interaction involves the insertion of a hydrophobic ephrin "G-H loop" into the ephrin-binding domain of the receptor. When two Eph monomers are bound to one ligand pair, they also form low affinity interactions through the H-I loop with the second ephrin in the ligand pair (Himanen et al. 2001; Smith et al. 2004). These interactions result in the dissociation of the ligand pair and allow four molecules to reorient to form a tetramer complex consisting of two Ephs and two ephrins. The tetramer complex adopts a ring like structure (Fig. 1-3 B) in which both receptors bind to both ligands. The formation of the tetramer places the two Eph receptors in a position to phosphorylate each other on key tyrosine residues (Fig. 1-3 C). The phosphorylation of tyrosine residues causes major conformational changes and release the inhibition on the receptor kinase activity.

After receptor-ligand tetramers form, they sometimes further cluster into bigger aggregates, which consequently amplifies activation. The aggregation process relies on both the extracellular and the juxtamembrane domains of Eph receptors and ephrins (Himanen et al. 2001; Himanen and Nikolov 2003a). The SAM domain of Eph receptors is also involved. Evidence shows that it is the high degree of clustering that explains the activation of Eph receptors that are not bound to ephrins (Wimmer-Kleikamp et al. 2004). We found in our experiments that the overexpression of EphA3 receptor alone in fibroblast cells leads to some level of phosphorylation and activation of the receptors 
even in the absence of ligand stimulation, likely through a similar mechanisms (data not shown).

\section{Signaling transduction after Eph activation}

Following Eph activation, a complex signaling network comprised of multiple signaling cascades is turned on. Similar to other RTKs, Eph receptors bind to signaling proteins and form substantial receptor/effectors protein complexes. To date, an assortment of signaling proteins for Eph receptors has been identified (Pasquale 1997; Klein 2004; Pasquale 2005). Among them, many are adaptor proteins that have unique roles in the signal transduction process. These adaptor proteins possess a number of protein docking sites and enable other effectors to dock near activated Eph receptors and the plasma membrane. They serve as the "scaffolds" and help to organize the receptor/effectors complexes (Birge et al. 1996; Buday et al. 2002). Inside the protein complexes, enzymes are activated through their association with the receptor and are able to catalyze reactions on their substrates efficiently. Consequently, downstream signaling pathways are turned on and various cellular phenotypes are expressed. This important topic will be discussed in more detail in Part III.

The activation of Eph/ephrin not only affects the state of downstream signaling pathways, it also has a direct effect on the localization of the receptors and their associated proteins. Previously, it has been shown that both Eph and ephrin molecules interact with PDZ domain proteins and membrane-bound receptors after activation. These interactions help to localize the receptor/effectors complexes to specialized membrane regions such as synapses or lipid rafts. Lipid rafts are small membrane domains where 
certain lipids (e.g. sphingolipids and cholesterol) and cytoplasmic enzymes (e.g. Src family kinases) are concentrated (Simons and Toomre 2000). Both the aggregation of Eph/ephrin molecules and the localization to lipid rafts or larger lipid patches are important events in Eph mediated signaling (Davy et al. 1999).

\section{Adhesion vs. repulsion}

Eph and ephrin signaling regulates both repulsive signaling and attractive signaling (Flanagan and Vanderhaeghen 1998; Zhou 1998; Drescher 2002; Knoll and Drescher 2002). It is intriguing to understand how the switch between adhesion and repulsion is modulated and what factors determine the different cellular responses.

First and foremost, the expression level of different effector proteins in different cell populations directly influences the outcome of Eph receptor activation in these cells. In addition, individual Eph receptors have varying binding affinity with certain effectors and therefore activate different signaling cascades. For instance, in hippocampal neurons, EphAs and EphB2 induce opposite effects in spine formation (Wilkinson 2000; Hindges et al. 2002a). These effects are likely mediated through two distinct groups of RhoGEF proteins that preferentially up-regulate RhoA or Rac1 respectively after activation of Eph receptors.

A second possible explanation for the dual effects stems from the different cell surface ephrin densities, which influence the aggregation level of the receptors. The resulting "receptor to ligand" ratio in the aggregates controls the activation of distinct binding proteins. Consequently, different cellular responses are observed. For example, high ephrin-B1 density induces the recruitment of LMW-PTP and Nck1 to EphB1 
receptors and up-regulate integrin signaling (Huynh-Do et al. 1999). It has been speculated that LMW-PTP down-regulates Eph activity because it dephosphorylates Eph receptors (Marte et al. 1997). A second line of evidence supports this hypothesis: EphA3 signaling at a low level of activation mediates attractive effects in retinal neurons but changes to repulsive effects as the receptors become further activated (Hansen et al. 2004).

Alternately, the expression of different Eph receptors on the same cells might affect how they collectively respond to ephrin stimulation. It is known that the truncated form of EphA7 dimerizes with wild type EphA7 and that dimerization is detrimental to normal signaling (Holmberg et al. 2000). A similar blocking effect was also noted on cells expressing Eph receptors and their cognate ligands (Dutting et al. 1999; Yin et al. 2004). For example, temporal retinal neurons express high level of EphA3 receptor and therefore project towards the anterior tectum where ephrin expression is low. The ectopic expression of ephrinA2 in these neurons down-regulates the repulsion induced by high level of ephrins on the tectum and allows them to project to a posterior location on the tectum. This finding suggests that the binding of receptors and ligands on the same cells takes place but lead to no activation of the receptors.

In addition, the interaction between Eph receptors or ephrins with non-effector proteins plays a role in determining cell responses. Research shows that RTKs such as PDGFR, FGFR or cytoplasmic enzymes (e.g. Src family kinases) have the ability to phosphorylate Ephs and ephrins (Bruckner et al. 1997; Chong et al. 2000). Thus, the activity level of these kinases has a significant impact on Eph receptor signaling in reaction to ephrin stimulation under different conditions. Furthermore, extracellular 
matrix proteins (ECM proteins) constitute another group of molecules that are involved in Eph mediated signaling. Interaction between Eph receptors with ECM protein receptors explains why ephrin-A5 induces opposite effects on neurons growing over laminin versus fibronectin (Gu and Park 2001; Weinl et al. 2003).

Receptor-ligand cleavage and endocytosis are also possible mechanisms through which the switch from adhesion to repulsion takes place. After the interaction of Eph receptors and ephrin ligands on two cells takes place, the high affinity receptor-ligand binding is difficult to uncouple. Consequently, attractive responses such as cell adhesion or cell migration are likely to be observed. However, when ADAM proteases are present in the extracellular cleft, they recognize the receptor-ligand tetramers and catalyze the proteolysis of the receptors [Fig. 1-3 D, (Hattori et al. 2000; Mancia and Shapiro 2005)]. Interestingly, ephrins are also substrates of the serine protease, rhomboid, which releases the ligands from cell membrane after proteolysis (Pascall and Brown 2004). Similar to other RTKs, the signaling complex is endocytosed and degraded following receptor or ephrin cleavage, [Fig. 1-3 E, F, (Hattori et al. 2000; Marston et al. 2003; Zimmer et al. 2007)]. Evidence exists to show that the cleavage and endocytosis of the bound Eph receptors or ephrins are critical to repulsive phenotypes such as the dissociation of Eph and ephrin expressing cells, cell detachment, and cell sorting. For example, when ephrinB expressing dorsal retinal neurons are stimulated with EphB2, endocytosis takes place in the reverse direction and the growth cones of these neurons collapse (Mann et al. 2003). The endocytosis of ephrins is necessary for the growth cone collapse to take place. The RhoGEF protein, Vav2 may be involved in this process since Vav knockout animals have major defects in Eph endocytosis and the neurons from these animals display less growth 
cone collapse in response to ephrin treatment (Cowan et al. 2005). Another protein, C$\mathrm{Cbl}$ seems to be also important in the internalization of at least some Eph receptors as well (Walker-Daniels et al. 2002; Sharfe et al. 2003). When EphA2 expressing tumor cells were stimulated by ephrin-A1, endocytosis and degradation followed in a C-Cbl dependent way. Similarly, EphA3 activation by ephrin-A1 induces C-Cbl association with Crk adaptors and its phosphorylation by Src kinase. These changes lead to EphA3 degradation at the end. It is necessary to point out that receptor proteolysis and endocytosis are also effective methods to terminate Eph signaling and probably take place at the end of attractive signaling as well.

\section{Eph/ephrin signaling is unique among RTKs}

Eph/ephrin signaling has several unique characteristics. First, Eph receptors are only activated by membrane-bound ligands but not soluble ligand monomers. When soluble forms of the ligands are used, artificial cross-linking is usually required to dimerize the ligands before application (Davis et al. 1994). This characteristic is different from how other RTK receptors interact with their ligands. Second, when Eph receptors and their cognate ligands are expressed on the same cells, sometimes they form receptor-ligand complexes that do not transmit signals effectively (Yin et al. 2004). This cis-interaction may be a mechanism to block functional Eph receptor signaling, as we pointed out earlier (Dutting et al. 1999). In addition, both A and B subclass ephrins are able to mediate downstream signaling in spite of their lack of kinase activity (Mann et al. 2003; Davy et al. 2004). Signal transduction through the ligands is termed "reverse signaling" and has not been found on other RTK ligands (Grunwald et al. 2001a; Davy and Soriano 2005). 
Lastly, although most of the functions of Eph receptors depend on the kinase activity of the receptors, kinase-independent signaling also exists (Wang et al. 1999; Matsuoka et al. 2005; Miao et al. 2005). We will discuss some of these important topics in detail in another chapter.

\section{Part II. Functions of Eph and ephrin signaling}

The wide expression pattern of Eph receptor or ephrin signaling is in accordance with the wide range of functions modulated by them. To this date, Eph receptors have been implicated in cellular events such as axon guidance, topographic projection, tissue patterning, cell sorting, cell migration, synapse regulation, angiogenesis, tumorigenesis and more. In the following section, we discuss some representative examples of these functions, especially in the nervous system.

\section{Axon guidance}

The development of the nervous system requires accurate neuronal innervations of targets over a long distance in a complex environment. The precise connections of the neural network are necessary for the nervous system to function. However, the mechanisms for the connections to form remained elusive for many years until Dr. Sperry first proposed the "chemoaffinity theory" in the 1940s. Through extensive research in fish and frogs, Dr. Sperry discovered that the neural circuits were indeed hardwired early in development and the expression of specific molecular tags on the neural cells determined their fate. Therefore, it has been proposed that receptors on the navigating 
cells, through recognizing specific molecular tags on target cells, regulates precise neural innervations (Sperry 1950; Sperry 1951). In later studies these molecular tags were further characterized based on their sub-cellular localization and effects: membranebound attractive agents, membrane-bound repulsive agents, diffusible attractive agents and diffusible repulsive agents (Tessier-Lavigne 1995; Tessier-Lavigne and Goodman 1996). The ligands for Eph receptors fall in the categories of membrane-bound attractive or repulsive agents. For instance, they have been implicated in guiding neuronal axons across the midline and modulating the formation of the hippocampal-septal projection (Cheng et al. 1995; Drescher et al. 1995; Flanagan and Vanderhaeghen 1998; Wilkinson 2001; Yue et al. 2002).

A well-characterized system in which Eph/ephrin signaling plays a pivotal role is the retinotectal projection (Fig. 1-4). Axonal projections from the retina to the superior colliculus (SC) in mammal or optic tectum in chicken, frog and fish are topographically established during development. An inspection of the expression pattern of various Eph receptors and ligands discloses that along both $\mathrm{A} / \mathrm{P}$ axis and $\mathrm{D} / \mathrm{V}$ axis, graded expression pattern exists [(Drescher 1997; Monschau et al. 1997), Fig. 1-4 A]. In the retina, a high temporal to low nasal gradient of EphA3 and EphA5 expression is noted, while a high posterior to low anterior gradient of ephrin-A2 and ephrin-A5 is found on the chick tectum. Temporal retinal neurons, with high expression of EphA receptors, are sensitive to ligand-induced repulsion. As a result, they are repelled from the posterior tectum and project towards the anterior tectum instead, where low ligand concentration is encountered. In comparison, the nasal axons with low EphA expression venture into the high ligand expressing posterior tectum as a low level of ephrin stimulation has been 
found to be attractive (Hansen et al. 2004). In addition, there is a uniform expression of EphA4 and a high nasal, low temporal expression of ephrin-A ligands in the retina as well. The retinal expression of ephrin-As, in a gradient opposite to the EphA receptors is found to sharpen the EphA gradient by exerting a blocking effect on EphA signaling (Knoll and Drescher 2002; Yin et al. 2004). Similar to the receptor/ligand expression along the $\mathrm{A} / \mathrm{P}$ axis, graded expression of $\mathrm{EphB}$ receptors and ephrin-Bs exists along the D/V axis. The interaction between EphBs and ephrin-Bs regulates the topographic establishment of projection between the retina and the tectum along this axis (Drescher et al. 1997; Mann et al. 2002).

Studies have shown that when EphA3 is ectopically expressed in retinal neurons, these neurons are inclined to project to an anterior position whereas other neurons shift posteriorly to fill in the space (Brown et al. 2000). Furthermore, in ephrin-A5 null mice, the temporal retinal axons overshoot into the posterior SC, confirming the importance of ephrin-induced repulsion in the formation of the projection. Interestingly, nasal axons shift anteriorly to fill the space left empty by the temporal retinal neurons in these mice (Frisen et al. 1998; Feldheim et al. 2000). A competition mechanism was therefore proposed to explain these findings: the temporal and nasal retinal neurons compete to innervate the SC and their ability to do so largely depends on their sensitivity to repulsion.

Ephrin-Bs are also able to mediate axon guidance through reverse signaling. For instance, EphB2 null mice were found to have severe defects in the projection of the posterior part of the anterior commissure of the cerebellum, the acP tract. The axons in the tract no longer extend across the midline like in wild type animals. Instead, they 
project ventrally (Henkemeyer et al. 1996). An analysis of the expression pattern reveals that acP tract neurons express ephrin-B2 while tissues underlying the acP tract express EphB2. This expression pattern indicates that the reverse signaling through ephrin-B2 is critical in the axon guidance of the acP tract axons. In some studies, the expression of a truncated EphB2 gene without kinase activity was able to rescue the EphB2 null phenotype, further confirming the significance of ephrin-B reverse signaling (Cowan et al. 2004).

\section{Axon branching}

During the establishment of topographic mapping of retinal axons, the formation of collateral branches from axonal shafts is a critical event. It is observed that usually retinal axons grow into the posterior region of the tectum past their final destinations. Although these axons originally overshoot their target sites, collateral branches extend into their future termination zones, preferentially towards where future synaptic connections form (Fig. 1-4 A). Later, the growth cones retreat to their final termination zone and form synapses, while all ectopic branches disappear when the topographic projection is complete [(Pasquale 2005), Fig. 1-4 B].

Eph forward signaling is involved in the inhibition of branching beyond the final termination zone through repulsion induced by high ephrin-A2/-A5 in the posterior tectum (Yates et al. 2001; Sakurai et al. 2002). To further support this finding, in vitro experiments have shown that temporal neurons preferentially branch over anterior tectal tissues (Yates et al. 2001). However, when EphA3-Fc was applied to block ephrin function in the tectal tissues, the temporal neurons branched equally over anterior or 
posterior tectal membranes. Moreover, the Eph/ephrin signaling is also prominent in determining the direction of collateral branch extension within the termination zone. Ephrin-B1 is expressed in a high medial to low lateral gradient in the SC (Hindges et al. 2002b). Similar to the dual effects ephrin-A5 has on retinal axons along the A/P axis (Gao et al. 1999), ephrin-B1 at a low concentration attracts branches in a medial direction but guides the branches in a lateral direction through repulsion at a high concentration.

\section{Axon fasciculation and defasciculation}

In general, when axons originate from the same areas and project to neighboring sites, they often form bundles and extend in the same direction (Yates et al. 2001). Eph/ephrin signaling plays a critical role in fasciculation. For example, in vitro assays have demonstrated that ephrin A5 promotes the fasciculation of EphA expressing cortical axons. Fasciculation allows most axons to follow the pioneer axons and navigate efficiently to their targets. Indeed, it has been reported that axons that follow usually possess smaller and less elaborate growth cones compared to the pioneer axons (Wilson and Easter 1991; Caras 1997). Near the destination sites, the axon bundles undergo defasciculation to allow individual axons to exit and reach their target neurons. EphB receptors play major parts in regulating the de-fasciculation of hippocampal neurons (Chen et al. 2004).

\section{Spine formation}

During development, neuronal dendrites extend filopodia towards axons of connecting neurons. Once contact is made, the dendritic filopodia transform into 
dendritic spines, which contain post-synaptic termini of the synapses (Murai and Pasquale 2004). The dendritic spines have a distinct mushroom-like appearance and an elaborate internal structure. The enrichment of neurotransmitter receptors on spines allow stimulus relay from the pre-synaptic termini to post-synaptic termini to take place. Formation of dendritic spines is a process that requires both Eph and ephrin functions. As reported by Penzes et al, after activation of EphB receptors in hippocampal neurons, RhoGEF Kalirin activates Rac1 and downstream effector PAK. The up-regulation of Rac1 activity is found to be necessary to spine formation (Ethell et al. 2001; Penzes et al. 2003). In addition, the clustering of Eph receptors promotes the spine maturation process via the interaction with proteins such as Syndecan-2 and PDZ domain containing proteins like PICK1, GRIP, Syntenin (Torres et al. 1998; Bruckner et al. 1999; Knoll et al. 2001). Indeed, syndecan-2 phosphorylation after EphB2 activation has been found to be adequate in inducing spine formation (Ethell et al. 2001).

Furthermore, Eph receptors are also involved in synaptic plasticity. Grunwald et al observed that EphB2 interacted with NMDA type glutamate receptors and regulated synaptogenesis (Grunwald et al. 2001a). Indeed, EphB2 induces the Src kinase mediated phosphorylation of NMDA receptor and increases calcium influx mediated by NMDA receptors in response to glutamate stimulation (Takasu et al. 2002a). In EphB2 null animals, long-term potentiation (LTP) and synaptic depression are both severely compromised, confirming the involvement of Eph signaling in spine function and synaptic plasticity. 


\section{Cell proliferation and differentiation}

Eph/ephrin signaling affects cell proliferation and cell fate. During development, the injection of ephrin-A-Fc and EphA-Fc into the sub-ventricular zone increases cell proliferation by inhibiting the endogenous Eph-ephrin signaling (Conover et al. 2000). It is therefore speculated that $\mathrm{Eph} / \mathrm{ephrin}$ activation regulates the size of brain by inducing apoptosis (Depaepe et al. 2005). In vitro treatment of cultured cortical progenitors confirms the speculation since soluble ephrin-A ligands induce rapid cell death in a caspase-3 dependent way. Furthermore, ephrin-A2 knockout animals have a larger than usual olfactory bulb, suggesting that ephrin-A2 mediated signaling negatively regulates cell proliferation (Holmberg et al. 2005). Similarly, in EphA7 loss-of-function mutant animals, a two-time decrease of cell death and a larger size brain were observed (Depaepe et al. 2005).

Eph signaling is also implicated in the differentiation of a variety of cells including human hematopoietic cells, stem cells, and retinal cells (Suenobu et al. 2002; Moore et al. 2004). The role of Eph signaling in neural cell fate is just beginning to be understood. For example, the forward signaling of EphA receptors promotes cell differentiation of neural precursor cells in a MAPK dependent fashion (Aoki et al. 2004).

\section{Tissue patterning}

The developing vertebrate hindbrain is characterized by a series of individual segments called "rhombomeres". Within each segment, distinct sets of genes are expressed. The segmentation of the hindbrain and the maintenance of the boundaries between two adjacent rhombomeres are important events in the hindbrain development. 
Insights on how Eph signaling is involved in these processes were gained when it was discovered that loss of Eph signaling led to the disruption of restricted gene expression pattern in the segments (Xu et al. 1995). The expression of Eph receptors and ephrin in the zebrafish hindbrain accords with such a role: EphA4, EphB2 and EphB3 are expressed in rhombomeres $\mathrm{r} 3$ and $\mathrm{r} 5$ while ephrin-B1, -B2 and -B3 are expressed in $\mathrm{r} 2$, r4 and r6 (Henkemeyer et al. 1994; Gale et al. 1996a). When ectopic expression of either ephrin-B2 or EphA4 was induced in developing zebrafish embryos, it was found that ephrin expressing cells were sorted to the edges of Eph expressing segments and Eph expressing cells were excluded from ephrin expressing segments (Mellitzer et al. 1999; $\mathrm{Xu}$ et al. 1999). Thus, both forward signaling and reverse signaling are required for this cell sorting process to take place.

\section{Cell migration}

The migration of neural crest cells is an important event that gives rise to spinal and autonomic ganglia. This process requires forward signaling through EphB receptors and EphA4 receptor in mice (Smith et al. 1997; Wang and Anderson 1997). In vitro assays revealed that ephrin-B ligands had the ability to control the migration of neural crest cells (Krull et al. 1997; Wang and Anderson 1997). In ephrin-B2 null embryos, the migration of neural crest cells follows a more scattered route compared to in wild type mice (Smith et al. 1997). Since a truncated ephrin-B2 without cytoplasmic segment rescued the phenotype, it was proposed that the reverse signaling through ephrin-Bs was not required. 


\section{Angiogenesis}

Angiogenesis is the formation of blood vessels through remodeling of a primitive endothelial network during embryogenesis. The functional significance of Eph and ephrin molecules in angiogenesis was first demonstrated when ephrin-B2 deficiency in embryos was found to lead to a disrupted vascular system (Wang et al. 1998). It has also been shown that the activation of EphB receptors induces the formation of a primitive endothelial network (Daniel et al. 1996; Stein et al. 1998b). Furthermore, EphB4 and ephrin-B2 are expressed in the veins and arteries respectively (Wang et al. 1998; Gerety et al. 1999; Adams and Klein 2000). Repulsive interactions induced by Eph-ephrin signaling may help to create the arterial-venous boundaries. In addition, downstream signaling of Eph receptors and ephrins also regulates cell adhesion through integrin signaling, which is important in helping the vascular network to settle in different tissues (Huynh-Do et al. 1999).

\section{Tumorigenesis}

The initial identification of high expression of Eph molecules in cancer cell lines intrigued researchers, and led to studies of their role in tumorigenesis. Today, almost all Eph receptors and ephrin ligands have been found to display unusual expression pattern in tumor tissues, strongly suggesting a direct involvement of these proteins in tumor development. For example, EphA2 receptor is up-regulated in prostate tumors, colorectal tumors, lung tumors and melanomas and the level of up-regulation corresponds to the stage of tumor progression and metastasis (Easty et al. 1999; Ogawa et al. 2000; Kinch and Carles-Kinch 2003). Furthermore, Zelinski et al reported that overexpression of 
EphA2 was enough to induce malignant transformation in mammary epithelial cells. More importantly, though the kinase activity of the receptor is critical in some cases, receptor activation takes place in a ligand independent way due to the clustering of the receptors (Zelinski et al. 2001; Fang et al. 2005). These observations all suggest that Eph receptors directly promote tumorigenesis at least in some cases.

Surprisingly, studies on the role of Eph receptors in tumorigenesis have also led to conflicting results. For instance, the loss of EphB receptors in colorectal cancer is strongly correlated with the state of cancer progression (Batlle et al. 2005). Similarly, EphA1 was found to be significantly down-regulated in two kinds of non-melanoma skin cancers (Hafner et al. 2006). In addition, ephrin-B and ephrin-A activation have been shown to suppress cell proliferation induced by neurotrophins, probably through the activation of PI3K/PDK1/Akt pathway (Tang et al. 2000a; Tang et al. 2000b).These findings seem to contradict other studies we discussed earlier and reveal the complex nature of Eph-ephrin signaling in tumor development. The dual effects of Eph-ephrin signaling in tumor tissues is therefore analogous to the signal transduction in the nervous system, where both attractive and repulsive responses are triggered by the same stimulation.

In addition to these studies which demonstrate that unusual expression of Eph receptors and ligands directly leads to tumor growth (Zelinski et al. 2001), evidence exists to suggest Ephs and ephrins contribute to tumor progression by regulating cell attachment and migration. Through regulating actin cytoskeleton reorganization and integrin signaling, the activation of Eph receptors increases the metastatic potential of melanomas and mammary tumors (Andres et al. 1994; Vogt et al. 1998). 
Based on these works, numerous research groups have attempted to reverse tumor progression through disrupting Eph signaling and have achieved significant success (Brantley et al. 2002; Brantley-Sieders et al. 2004; Duxbury et al. 2004; Fang et al. 2005). These works will help to pave the road towards using Eph inhibition in cancer treatment.

\section{Part III. Signaling pathways downstream of activated Eph receptors and ephrins}

\section{Binding proteins of Eph receptors}

After ephrin stimulation, the phosphorylation of key tyrosine residues on Eph receptors or ephrins enables signaling molecules to interact with them (Kalo and Pasquale 1999). Activated signaling molecules turn on diverse downstream pathways, which eventually regulate the cellular events that we previously discussed (Kullander and Klein 2002). To date, more than 20 proteins have been confirmed to be directly associated with Eph receptors [Table 1-1, (Himanen and Nikolov 2003a; Huot 2004; Pasquale 2005)] and the signaling cascades they regulate are summarized (Fig. 1-5).

A large percentage of the identified binding proteins contain Src homology-2 domains (SH2), such as Src, Abl/Arg, Crk, Grb2, Nck1, SHP-2, PLC gamma, RasGAP, P85-PI3K (Pandey et al. 1995; Holland et al. 1997; Stein et al. 1998a; Zisch et al. 1998; Kalo and Pasquale 1999; Kullander and Klein 2002). Research on the properties of the $\mathrm{SH} 2$ motifs reveal that SH2 domains have high affinity binding for phosphorylated tyrosine residues and interact with the cytoplasmic domains of phosphorylated receptor tyrosine kinases (Chattopadhyay et al. 1999). It is important to point out that the SH2 
domains of different proteins exhibit distinct binding preferences, which largely depend on the three amino acids behind the phosphorylated tyrosine residue (Songyang et al. 1993). In addition, SH2 domain proteins often possess other protein binding domains or catalytic domains, allowing them to recruit other proteins into the receptor/effectors complex and regulate a wide range of signaling events such as actin cytoskeleton restructuring, MAPK activity modulation, and integrin signaling.

Another major group of binding proteins contain the PDZ domain. This domain interacts with the PDZ binding motif at the C-terminus of Eph receptors. Proteins with PDZ domains include Syntenin, Afadin-6 (AF-6), protein interacting with C kinase-1 (Pick1) and Glutamate receptor interacting protein (Grip) (Hock et al. 1998b; Torres et al. 1998; Buchert et al. 1999). The function of these proteins remains relatively unclear, though some studies suggest that they might assist in localizing Eph receptors or ephrins to specialized membrane regions such as synapses or lipid rafts. For example, research has shown that Pick-1 mediates the interaction between Eph receptors and other synaptic receptors and activates the PKC kinase cascade (Bruckner et al. 1999; Knoll et al. 2001).

Some signaling proteins interact with Eph receptors through domains other than $\mathrm{SH} 2$ or PDZ domains. These proteins include Ephexin, LMW-PTP, FAK (Stein et al. 1998a; Miao et al. 2000; Sahin et al. 2005).

\section{Eph signaling: Src family kinases and Nck1}

Several binding proteins have been implicated in the process of cytoskeleton restructuring. In response to ephrin stimulation and Eph activation, they set the cytoskeleton machinery in motion and lead to either repulsive or attractive responses in a 
context-dependent manner. Src family kinases such as Abl/Arg and adaptor protein Nck1 are good examples of these proteins.

$\mathrm{Abl}$ and Arg proteins are members of Src family kinases (SFK), a family of SH2domain-containing cytoplasmic tyrosine kinases. Like other SFK members, they phosphorylate a wide array of substrates including RTKs and cytoskeletal regulatory proteins. They are different, however, from other SFK members because they possess a F-actin-binding motif at the $C$ terminus (Robinson et al. 2000; Hernandez et al. 2004). $\mathrm{Abl} / \mathrm{Arg}$ proteins are co-expressed with Eph receptors in the brain and are mainly localized on the growth cones, indicating that Abl/Arg proteins may play significant roles in Eph mediated pathfinding (Salgia et al. 1997). Indeed, the Abl/Arg kinases associate directly with the juxtamembrane domain of Eph receptors through their $\mathrm{SH} 2$ domain upon ephrin-B1 stimulation. Surprisingly, this binding leads to a decreased kinase activity of Abl/Arg (Yu et al. 2001). In addition to the juxtamembrane domain binding, Abl/Arg also interact indirectly with Eph receptors through Crk and other Src family kinases (Wang et al. 1996; Plattner et al. 1999). Since Abl/Arg have been found to have a promotional role in neurite outgrowth (Zukerberg et al. 2000), it is possible that the stabilized association of Abl/Arg to Eph receptors serves to sequester Abl/Arg kinases from their substrates and leads to the downregulation of Abl/Arg function. A second line of evidences shows the functional absence of Abl/Arg in the knockout animals result in serious defects of the nervous system, which bear striking resemblance to ephrin-A5 knockout animals (Koleske et al. 1998; Holmberg et al. 2000).

Other SFKs are involved in cytoskeleton regulation as well. Src kinase is known to phosphorylate a range of proteins involved in focal adhesion or cytoskeleton activity, 
such as cortactin, tensin, paxillin, p130Cas and FAK (Burridge et al. 1988; Huang et al. 1997; Parsons and Parsons 1997). Through the phosphorylation of these important substrates, SFKs exert direct regulatory effects on cytoskeleton dynamics. For instance, the binding of Src kinases and FAK leads to the phosphorylation of both proteins and the activation of MAPK pathway (Guan 1997). Cortactin, a F-actin binding protein, causes F-actin bundles to form. Its phosphorylation by SFKs causes a reduction of its crosslinking ability.

Nck1 also plays a prominent role in the regulation of cytoskeleton restructuring. The majority of Nck1 binding proteins we know so far have been implicated in cytoskeleton regulation. Among them, p21 activated kinase (PAK), Wiskott Aldrich syndrome protein (WASp), Nck1 interacting protein (NIK) and Nck1 associated protein 1 (Nap-1) are best characterized (Bagrodia et al. 1995; Rivero-Lezcano et al. 1995; Ruan et al. 1999). PAK proteins, WASp/WAVE proteins and Nap-1 have all been found to bind to Rho family GTPases, which are directly involved in regulating actin-based cytoskeleton (Nobes and Hall 1995; Hummel et al. 2000). Furthermore, when PAK or NIK is each mutated, the resulting phenotype resembles the phenotype of Nck1 knockout animals (Ruan et al. 1999). The function of Nck1 was confirmed through studies on Drosophila Nck1 homolog Dreadlocks (Dock) (Garrity et al. 1996; Desai et al. 1999). Without the expression of Dock, it has been shown that axons of photoreceptor neurons no longer stop at the correct targets. Since Nck1 is the focus of our research, its role in Eph receptor signaling will be discussed later in more detail. 


\section{Eph signaling: Ras/MEK/ERK pathway}

Ras is a small GTPase that was first found in tumor cells. It mediates important signal transduction from cell surface RTKs to downstream pathways that regulate cell proliferation, transcription, and cytoskeleton modulation. Normally, active Ras molecules bind and activate Raf family kinases. Active Rafs then stimulate the mitogen activated extracellular signal regulated kinase activating kinase (MAPKK or MEK) family members. As the name suggests, these kinases phosphorylate and activate MAPK members [ERK1 and ERK2, (p42/44)]. The active ERK proteins are capable of phosphorylating many cytoplasmic substrates and regulating gene transcription, cell cycle, cell adhesion and cell mobility (Howe et al. 2002; Roux and Blenis 2004; Viala and Pouyssegur 2004).

Ras regulated pathway is critical in signal transduction downstream of Eph receptors. Several identified binding proteins of Eph receptors have been implicated in this pathway and the best understood one is Ras small GTPase guanine nucleotide activating protein (RasGAP) (Holland et al. 1997; Elowe et al. 2001). A SH2 domain containing protein, RasGAP interacts directly through both the juxtamembrane domain and the kinase domain with EphB1 receptor in a kinase dependent way (Hock et al. 1998a; Zisch et al. 2000; Elowe et al. 2001). Alternately, RasGAP has been found to interact with EphB2 indirectly through the mediation of p62dok (Holland et al. 1997). RasGAP converts GTP bound to Ras to GDP and renders the Ras protein inactive. Therefore, when RasGAP is brought in close proximity of Ras molecules localized near the plasma membrane by binding with Eph receptors, ERK1/2 activity is down-regulated. The down-regulation of ERK1/2 following EphB activation has been confirmed to be important in neurite 
retraction, growth cone collapse, and other repulsive signaling events (Tong et al. 2003). In addition, down-regulated ERK activities triggered by Eph activation explains why Eph receptors, compared to other RTKs, do not have a strong mitogenic effect on cell cultures (Brambilla and Klein 1995; Bruce et al. 1999).

Opposite to the function of RasGAP, multiple SH2 adaptor proteins such as Crk, Grb2, and Nck1 associate constitutively with Sos, a Ras-guanine nucleotide exchange factor (RasGEF). Once Eph receptors become activated, these adaptor proteins associate with phosphorylated Eph receptors through their SH2 domains and recruit Sos (Stein et al. 1996; Hock et al. 1998a; Stein et al. 1998a). By catalyzing the exchange of GDP to GTP on Ras, Sos is able to activate Ras and turn on the MAPK pathway (Hu et al. 1995; Byrne et al. 1996). This pathway might be the underlying mechanism of Eph mediated tumorigenesis observed in some studies.

Together, these observations show Eph activation either up-regulate or downregulate Ras/MEK/ERK pathway, depending on the signaling proteins present. It would be of great interest to understand how the interactions of RasGAP, Grb2, Nck1 and Crk with Eph receptors are differentially regulated, and if these differences account for different ephrin induced phenotypes.

The PDZ domain protein AF-6 is a Ras binding agent (Hock et al. 1998b). Through binding with Eph PDZ motif at the C-terminus, AF-6 might play a role in regulating Rasactivated MAPK pathway. Additionally, activated Ras/Raf/MAPK pathway is linked to the PI3 kinase pathway, important in cytoskeleton regulation, cell migration and cell adhesion (Hamada et al. 2003; Maekawa et al. 2003). Due to its importance in RTK signaling, PI3 kinase pathway will be described in the following section. 


\section{Eph signaling: PIP $_{2}$ related pathways}

PI3 Kinase associates with Ras family members and becomes activated when they are translocated in close proximity of the plasma membrane (Navolanic et al. 2003). The association of PI3K p85 subunit with EphA2 and EphA8 through the SH2 domain assists in this translocation and activation process (Pandey et al. 1994; Gu and Park 2001). Active PI3 kinase catalyzes the phosphorylation of phosphoinositide and the phosphorylated products bind and activate phosphoinositide-dependent protein kinase 1 (PDK1) and anti-apoptotic factor Akt kinases. The activation of PI3K/PDK1/Akt pathway has been implicated not only in the regulation of apoptosis and gene transcription (Navolanic et al. 2003) but also in integrin activity regulation, cell adhesion, and vesicle trafficking (Wennstrom et al. 1994; Shimizu et al. 1995; Carpenter and Cantley 1996). It has been shown PI3K plays a direct role on cell adhesion through direct modification of lipid molecules or alternately, $\mathrm{PI}(4,5) \mathrm{P}_{2}$, a substrate of PI3K regulates actin cytoskeleton through acting-binding proteins (Carpenter and Cantley 1996).

Phospholipase $\mathrm{C}$ gamma (PLC $\gamma$ ) is a primary effector that associates with EphB2 and EphA4 receptors via the juxtamembrane domain (Holland et al. 1997; Zisch et al. 1998). After associating with Eph receptors and being translocated to the plasma membrane, PLC $\gamma$ binds to $\mathrm{PI}(4,5) \mathrm{P}_{2}$ and converts $\mathrm{PI}(4,5) \mathrm{P}_{2}$ to diacylglycerol (Hall et al. 1996). Diacylglycerol regulates cytoskeleton restructuring through the activation of calcium channels and PKC (Clark and Brugge 1995). PKC in turn phosphorylates Raf kinase and contributes to the regulation of MAPK pathway (Navolanic et al. 2003). 


\section{Eph signaling: small GTPases}

As discussed in an earlier section, cytokskeletal rearrangement is an integral part of Eph mediated cell responses such as cell migration, neurite outgrowth, and growth cone collapse. Other than the proteins we discussed before, small Ras-like GTPases, Rac, cdc42 and RhoA, are also known to be key players (Dickson 2001). The activities of these three GTPases are major determining factors in actin cytoskeleton dynamics. Activated RhoA through its effector ROCK, leads to growth cone collapse and increased contraction (Dergham et al. 2002; Fournier et al. 2003). The inhibition of Rho activity, in comparison, reduces growth cone collapse rate (Wahl et al. 2000). Rac and cdc42, on the other hand, transduce signaling through p21 cdc42/Rac activated kinases (PAKs) and promote axonal outgrowth (Nikolic 2002; Sahin et al. 2005). They are also implicated in the formation of lamellapodia and filopodia, respectively (Nobes and Hall 1995). The activity of Rac and cdc42 is down-regulated by RhoA, possibly through competition for binding partners, since Rac inhibition induces effects that are similar to those induced by RhoA activation (Dickson 2001).

The PAK proteins are directly activated by Rac and cdc42. Eph receptor activation, however, plays a role in the activation of PAKs through adaptor protein Nck1. PAKs bind to Nck1 through the SH3 domains (Rivero-Lezcano et al. 1995; Quilliam et al. 1996). As Nck1 binds to activated EphB1 receptor through the juxtamembrane domain tyrosines, it helps to translocate PAKs from cytosol to the plasma membrane and brings the PAK proteins in close proximity of Rac and cdc42 (Bokoch et al. 1996). Consequently, PAKs are activated and able to phosphorylate their substrate proteins. 
Ephexin, a Dbl family GEF protein, was first identified through a yeast two-hybrid screen with the intracellular domain of EphA4 as the bait (Shamah et al. 2001). It interacts with the kinase domain of EphA receptors through its Dbl-homology-pleckstrinhomology domain (DH-PH) in a kinase independent way. As a Rho guanine nucleotide exchange factor (RhoGEF), ephexin activates Rho family GTPases. However, once EphA4 receptor becomes activated by ephrin-A1, ephexin is phosphorylated on the Tyr87 residue and the specificity of ephexin activity dramatically changes (Sahin et al. 2005). Instead of activating RhoA, cdc42 and Rac equally, ephexin preferentially activates RhoA activity, resulting in local growth cone collapse and morphological changes, phenotypes characteristic of RhoA up-regulation.

Another RhoGEF protein, Vav2 was identified recently as an EphA4 binding protein. Unlike ephexin, Vav2 is a Vav family guanine nucleotide exchange factor and contains a SH2 domain. After binding to phosphotyrosine residues on EphA4, Vav2 activates Rho small GTPases and induces growth cone collapse. Furthermore, as mentioned briefly earlier, endocytosis of EphA4, critical to growth cone collapse, may be regulated by Vav2 (Cowan et al. 2005).

Tiam1, a novel RhoGEF, interacts with both EphA2 and ephrin-B1. It up-regulates Rac activity preferentially. Dominant negative Tiam1 inhibits neurite outgrowth strongly, similar to the phenotype induced by overexpression of truncated forms of EphA2 or ephrin-B1 (Tanaka et al. 2004).

Other RhoGEFs, though not bound directly to Eph receptors, have been also implicated in the regulation of small GTPases downstream of Eph activation. Kalirin, for example, is a RhoGEF that specifically activates Rac1 after the activation of EphB2 
receptor (Penzes et al. 2001; Penzes et al. 2003). An immediate result of this activation is enhanced spine formation in hippocampal neuron cultures.

In addition, RasGAP regulates RhoGAP activity through direct binding at least in some cases (McGlade et al. 1993). RhoGAP inactivates RhoGTPases by activating the hydrolysis of GTP on Rho GTPses. Thus, the interaction between RasGAP and RhoGAP links Ras/MEK/ERK pathway to small GTPases.

\section{Eph signaling: cell adhesion}

Integrin signaling is an important signaling pathway that directly regulates the response of cells to extracellular matrix proteins. It is closely related to the MAPK pathway, as neuronal cell attachment to fibronectin activates MAPK through integrin signaling. PLC $\gamma$ activity may be responsible for this activation (Finkelstein and Shimizu 2000). EphB2 activation, on the other hand, causes a reduction of MAPK activity (Schlaepfer and Hunter 1998; Elowe et al. 2001) and has a regulatory effect on integrin signaling (Stein et al. 1998b).

Eph receptors exert their regulatory effects on integrin function through the modulation of R-Ras activity. A member of Ras small GTPase family, R-Ras activates PI3K but is not activated by Sos. Consequently, R-Ras does not activate Raf or the MAPK pathway (Marte et al. 1997). Instead, R-Ras is involved in the regulation of cell adhesion through integrin signaling. EphB2 receptors as well as Src family kinases phosphorylate R-Ras molecules on Tyr66 residue (Zou et al. 1999) and inhibit its binding with Raf kinase (Zou et al. 2002). RasGAP, on the other hand, regulates R-Ras activity by catalyzing the conversion of GTP bound to R-Ras to GDP. When RasGAP becomes 
associated with Eph receptors, it suppresses cell adhesion through R-Ras inactivation (Dail et al. 2006). As shown by Dail et al, regulation of R-Ras activity through Src kinase mediated phosphorylation or through RasGAP mediated inactivation are both important to ephrin-mediated responses. Whereas RasGAP is required to induce EphB2 expressing Cos- 1 cells to retract their peripheries after ephrin-B1 treatment, both RasGAP and Src kinase are critical to Eph mediated inhibition on cell migration. Moreover, growth cone collapse of primary cortical neurons also seems to require both mechanisms to downregulate the R-Ras activity. In addition to these regulations on R-Ras, SHEP-1 protein is involved in the R-Ras activity regulation as well (Dodelet et al. 1999). SHEP-1, a protein consisting of a SH2 domain and a RasGEF domain, interacts with R-Ras but does not activate it. Instead, it recruits R-Ras to the vicinity of activated Eph receptors and leads to R-Ras phosphorylation by Src family kinases.

Focal adhesion kinase (FAK) is another key player in integrin signaling regulation. Integrin-mediated focal adhesions are highly enriched with FAK and FAK is directly activated by integrins (Nobes and Hall 1995). FAK is found to be expressed in the brain and involved in the regulation of cell adhesion and cell migration (Carter et al. 2002; Beggs et al. 2003). Some studies suggest that FAK is able to negatively regulate axonal extension and dendritic spine in hippocampal neurons (Karanian et al. 2005; Moeller et al. 2006). FAK activity is regulated by Ephs and ephrins in several ways. EphA2 binds to FAK constitutively and Eph-associated Src kinases phosphorylate and activate FAK (Parsons and Parsons 1997). Paradoxically, FAK is dephosphorylated by SHP-2, a phosphatase that associates with the activated EphA2 receptors (Miao et al. 2000). Following dephosphorylation, inactive FAK dissociates from EphA2. Indeed, it has been 
noted that EphA2 activation in carcinoma cells results in the suppression of both FAK activity and integrin-mediated cell adhesion, indicating that FAK is dephosphorylated.

Eph receptor activation induces cell adhesion through integrin signaling (Stein et al. 1998b; Kikawa et al. 2002). In transfected human kidney cells, EphB1 activation elicits recruitment of LMW-PTP to the SAM domain and Nck1 to the juxtamembrane domain and leads to enhanced integrin $\alpha 5 \beta 1$ mediated cell adhesion. Another line of evidence suggests that the recruitment of LMW-PTP and Nck1 is an event that occurs in the presence of high density of ephrin ligands (Stein et al. 1998b; Huynh-Do et al. 1999; Kikawa et al. 2002). In addition, Nck1 interacting kinase (NIK) links Nck1 recruitment and integrin signaling, as the activation of NIK following association with Nck1 results in the activation of both c-Jun N-terminal kinase (JNK) and integrin (Becker et al. 2000).

Lastly, active PI3K was found to be required for integrin signal transduction in addition to regulating cell apoptosis and gene transcription. As Gu and Park reported, EphA8 regulates integrin signaling by interacting with PI3K in a kinase-independent way and helps localize PI3K to the plasma membrane near the integrin signaling complex $(\mathrm{Gu}$ and Park 2001).

\section{Reverse signaling through the ephrin-Bs}

As briefly discussed before, ephrin ligands have the ability to transduce signaling (Davy et al. 1999; Knoll et al. 2001; Mann et al. 2002; Davy and Soriano 2005). The phenomenon was first discovered when research showed that EphB2 receptors activated ephrin-B1 and ephrin-B2 and led to the phosphorylation of their tyrosine residues (Holland et al. 1996). The phosphorylated ephrin-Bs are capable of recruiting effector 
proteins and activating signaling cascades, similar to phosphorylated Eph receptors. This novel signaling was named "reverse signaling" as opposed to "forward signaling", which takes place through the receptors. In order to understand the effects of reverse signaling, new strategies are devised to discern reverse signaling from forward signaling. In these new strategies, wild type ephrins or Ephs are not eliminated from the system. Instead, they are replaced by mutated forms that lack the functional cytoplasmic region. These mutated ephrins or receptors are incapable of inducing downstream signaling events, and therefore enable the experimenters to dissect out and analyze reverse and forward signaling as two separate events.

Through these new approaches, it was found that reverse signaling by ephrin-Bs is implicated in many functions including axon guidance, cell sorting, cell migration (Henkemeyer et al. 1996; Wang et al. 1999; Xu et al. 1999). Similar to forward signaling, reverse signaling leads to either repulsive or attractive responses (Henkemeyer et al. 1996; Knoll et al. 2001; Kullander et al. 2001). The details of the mechanism are just beginning to be understood.

The intracellular domain of ephrin-Bs contains a PDZ motif at the N-terminus and is highly conserved. After being stimulated by Eph receptors, the ephrinBs undergo phosphorylation by cytoplasmic kinases such as Src family kinases, or activated RTKs such as PDGFR or FGFR (Holland et al. 1996; Bruckner et al. 1997; Chong et al. 2000; Palmer et al. 2002). The phosphorylated ephrin-Bs recruit effectors and adaptor proteins. The signaling proteins downstream of ephrins are summarized in Table 1-2 and the signaling cascades regulated by ephrins are shown in Fig. 1-6. 
Research shows five tyrosine residues of ephrin-B2, particularly tyrosine 304, are strongly phosphorylated upon Eph stimulation. The phosphorylation of ephrin-Bs mediates binding with downstream effectors directly, and also enables PDZ-RGS protein to dock on the PDZ motif (Su et al. 2004).

Nck2 has been found to associate ephrin-B1 in vitro through its SH2 domain (Cowan and Henkemeyer 2001). A member of Nck adaptor family, Nck2, is well-known in bridging effectors proteins to phosphorylated RTKs. Through its three SH3 domains, Nck2 interacts with several proteins including Abl-interacting protein-1 (Abi-1), a scaffold protein (Axin), and c-Cbl-associated protein (CAP). Interestingly, previous studies implicate all three of these proteins in actin cytoskeleton regulation, suggesting that Nck2 mediates ephrin-B signaling through regulating cytoskeletal rearrangements (Dai and Pendergast 1995; Shi et al. 1995; Ribon et al. 1998).

Though the mechanisms are not completely clear, it has been determined that the binding through the PDZ sequence is required in reverse signaling (Davy et al. 2004). For instance, PDZ-RGS, a PDZ binding protein and a regulator of heterotrimeric G protein signaling, links G protein coupled signaling to ephrin signaling (Lu et al. 2001; Lu et al. 2004). Other PDZ binding proteins including Syntenin, Pick1, Grip, Grip2, PHIP, and PTP-BL have been shown to interact with ephrin-Bs as well (Torres et al. 1998; Bruckner et al. 1999; Lin et al. 1999; Palmer et al. 2002). Some of these proteins such as Pick1, Grip1/2, and PHIP mediate synaptic localization of ephrins, while PTP-BL is involved in deactivating ephrin-Bs through dephosphorylation.

Recently, new progress has been made in identifying binding proteins for ephrin-Bs. Tiam1, a Rac guanine nucleotide exchange factor was found to associate with both 
ephrin-B1 and EphA3 and mediate neurite outgrowth (Tanaka et al. 2004). E14 cortical neurons expressing ephrin-B1 extend neurites on surfaces, which are coated with EphB2 and the activation of ephrin-B1 leads to Tiam1 activation. Expression of a dominant negative form of Tiam1 results in disrupted neurite outgrowth. Another newly discovered ephrin-B binding protein is Xenopus Dishevelled (Xdsh), a protein that up-regulates RhoA and Rho kinase (ROCK) activity (Tanaka et al. 2003). Xdsh interacts with ephrinB1 following its activation and mediates cell sorting through repulsion in the animal cap.

\section{Reverse signaling through ephrin-As}

The observation of reverse signaling through ephrin-As surprised researchers in the field because of the absence of cytoplasmic tails on A class ephrins. Convincing evidence, however, was presented with the discovery of four GPI-linked ephrin homologs and one Eph homolog (VAB-1) in C. elegans (Wang et al. 1999). Furthermore, it has been found the expression of VAB-1 in a kinase inactive form does not lead to a complete loss of function in mutant animals, suggesting that reverse signaling through the GPI linked ephrins must be important (Chin-Sang et al. 1999). This hypothesis is later confirmed by another study, in which mouse vermonasal neurons expressing high levels of ephrin-A5 project towards EphA6 expressing territory, suggesting an attractive effect of ephrin-A5 signaling (Knoll et al. 2001). The downstream signaling of ephrin-As remains unclear, though some studies suggest that Fyn tyrosine kinase is involved. It is likely that the interaction with other receptors through the extracellular domain is critical to ephrin-A mediated signaling. Indeed, integrin signaling is regulated by ephrin-A 
activation, possibly through direct coupling of the integrin molecules to ephrins (Davy et al. 1999; Wang et al. 1999).

\section{Summary}

Receptor tyrosine kinases turn on many cytoplasmic pathways after their activation. As members of the RTK superfamily, Eph receptor family and ephrins are highly expressed in a wide variety of tissues in developing and adult organisms. They have been found to play important roles in many developmental processes, including tissue patterning, angiogenesis, neural crest cell migration, axon fasciculation, axon guidance, and topographic neural map formation. In addition to the roles during development, Eph receptors have been shown to regulate adult functions ranging from learning and memory, to inducing pathological conditions such as tumorigenesis. Eph receptors interact with their membrane bound ligands and form 2 receptors:2 ligands tetramer complexes. Furthermore, these complexes are known to achieve a high level of clustering and become localized to specific regions such as lipid rafts or synapses. Depending on the receptors/ligands involved and the cellular environment, Ephrin-Eph interaction produces either repulsive effects or attractive effects. Although most of the functions depend on the kinase activity of the receptors, both kinase independent signaling through receptors and reverse signaling via ephrin ligands exist.

Some insights have been gained on how Eph receptors and ephrin ligands mediate different signaling events. A group of signaling proteins has been identified, many of which possess SH2 domains or PDZ domains. They function to link activated Eph 
receptors and ligands to major downstream signaling pathways such as Ras/MEK/ERK pathway, small GTPases, cytoskeleton, integrin signaling, $\mathrm{PIP}_{2}$ pathway.

However, the complexity of Eph and ephrin signaling remains elusive. Intriguing questions remain, in particular about how the same receptors induce opposite effects in different populations of cells or under different circumstances. The switch between repulsion and adhesion has not yet been elucidated, even though many hypotheses exist. Furthermore, the diversity of functions and the multitude of signal transduction pathways suggest that more binding proteins in the signaling cascades have yet to be discovered. It is imperative to explore how signaling proteins induce different outcomes in response to ligand stimulation. In addition, Eph/ephrin signaling has been implicated in tumorigenesis, especially in metastasis. Our understanding of these functions will therefore enable us to use Eph/ephrin targeted therapies in treating cancer and other diseases.

We started the project with a large-scale screen for strong interacting proteins with Eph receptors. Several novel binding proteins were identified, among which Nck1 was the strongest interactor. Because Nck1 is recognized as a key player in RTK signaling but has never been implicated in EphA mediated signaling, we set out on an in-depth study of the role of Nck1. Both fibroblast cell line and primary neuron cultures are used in this study. 


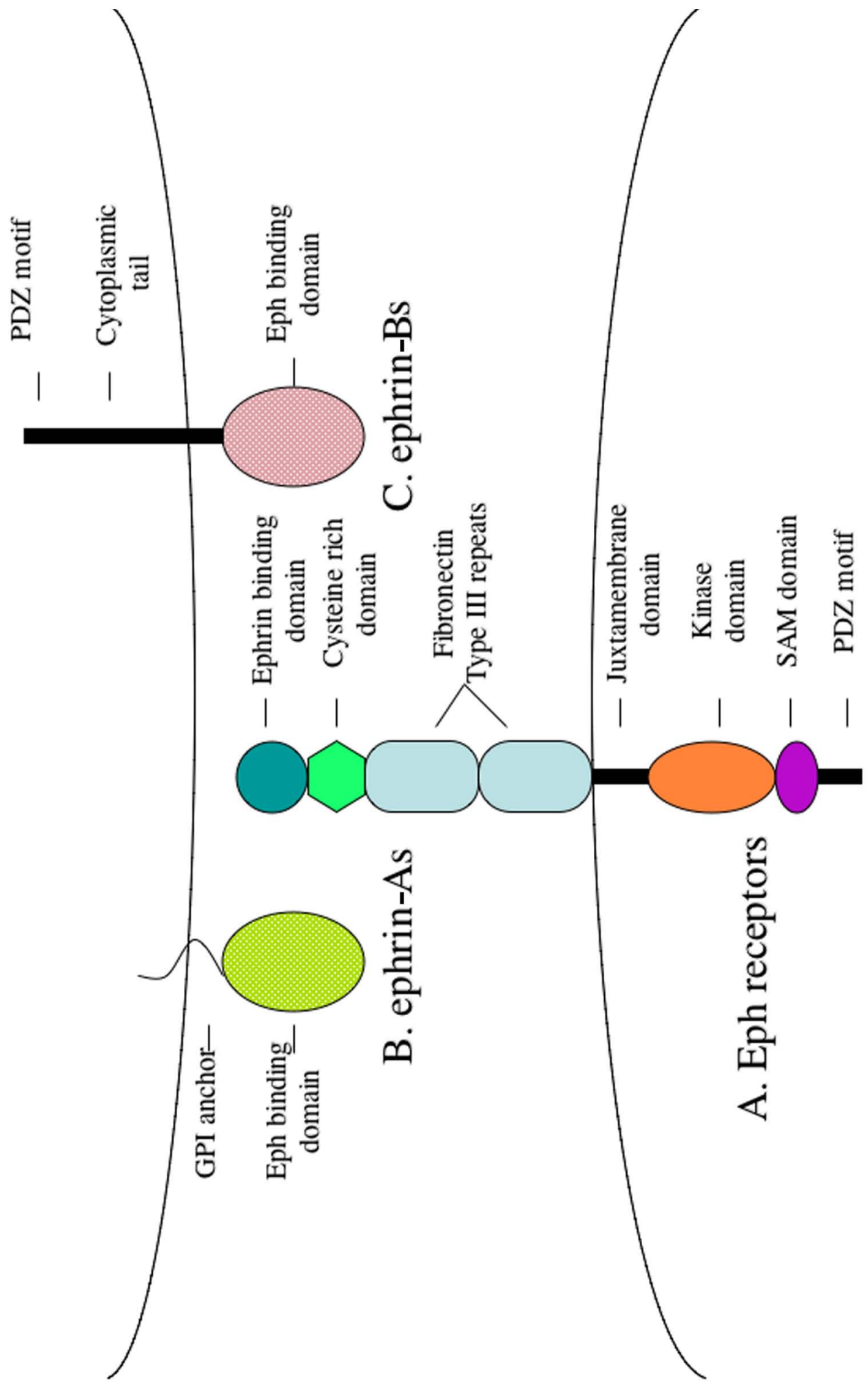


Figure 1-1. Domain structure of Eph receptors and ephrin ligands

(A) Domain structure of Eph receptors. They contain a globular domain, a Cysteine-rich domain, two Fibronectin III domains, the juxtamembrane domain, a tyrosine kinase domain, a SAM domain, and a PDZ binding motif. (B) Domain structure of ephrin-As. They connect to the cell surface through a GPI link. (C) Domain structure of ephrin-Bs. They contain an extracellular domain, a transmembrane domain, and a cytoplasmic tail. 
A.

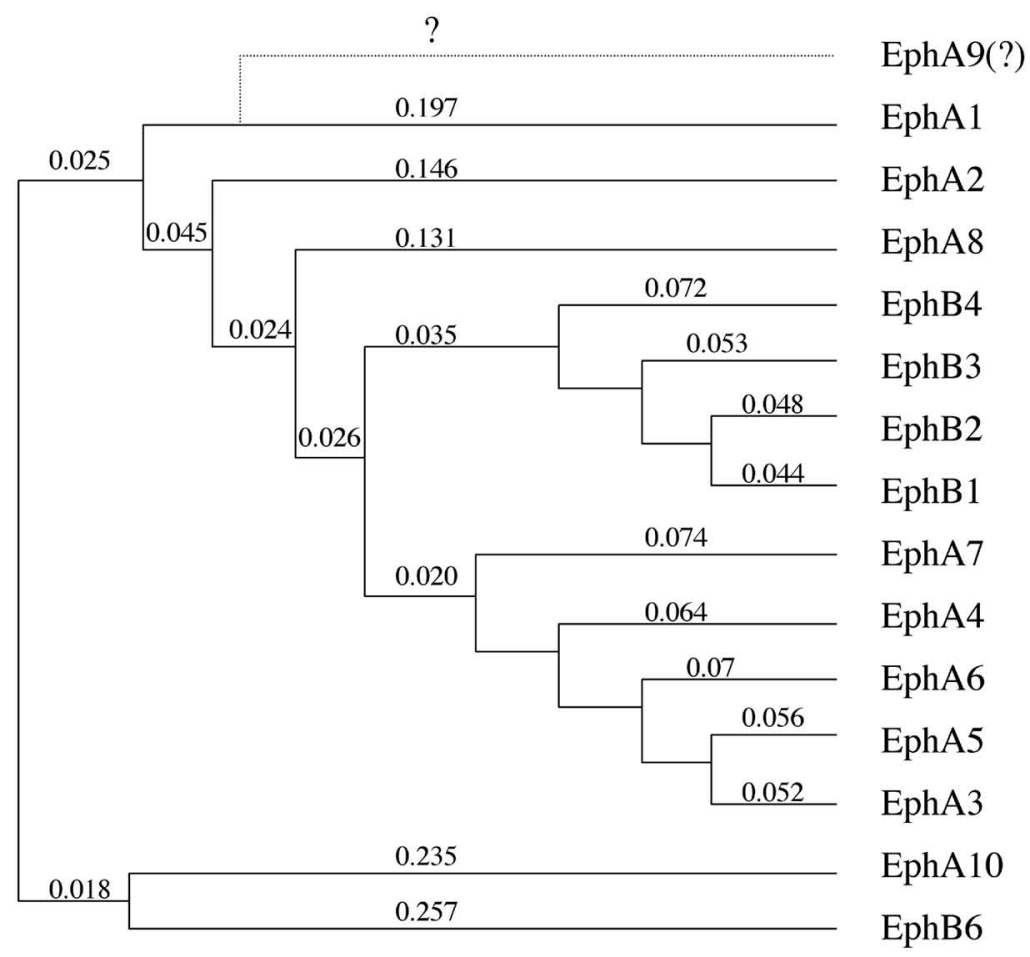

B.

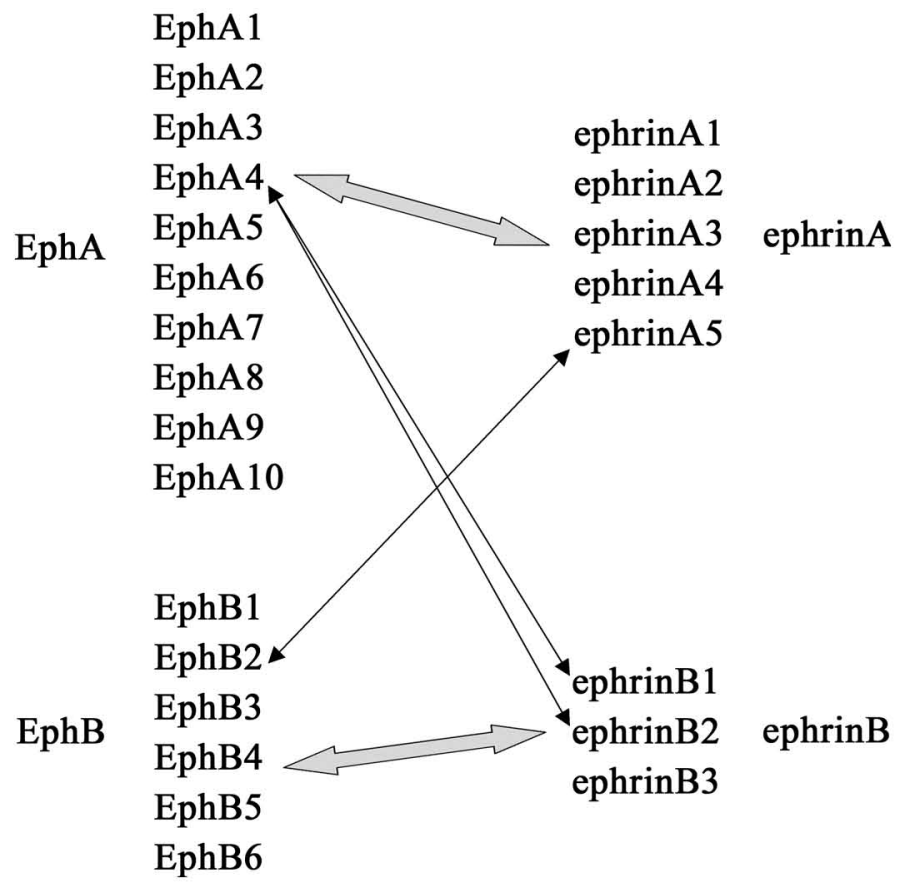


Figure 1-2. Phylogram of Eph family kinases and class-specific interactions between Eph receptors and ephrins.

(A) A phylogram of Eph receptors, modified on the basis of The protein tyrosine kinase family of human genome (Robinson et al, Oncogene, 2000). The evolutionary distance was calculated based on the sequence homologies of the kinase domain. (B) Receptorligand interaction is usually subclass-specific but binding across subclasses sometimes occurs. 


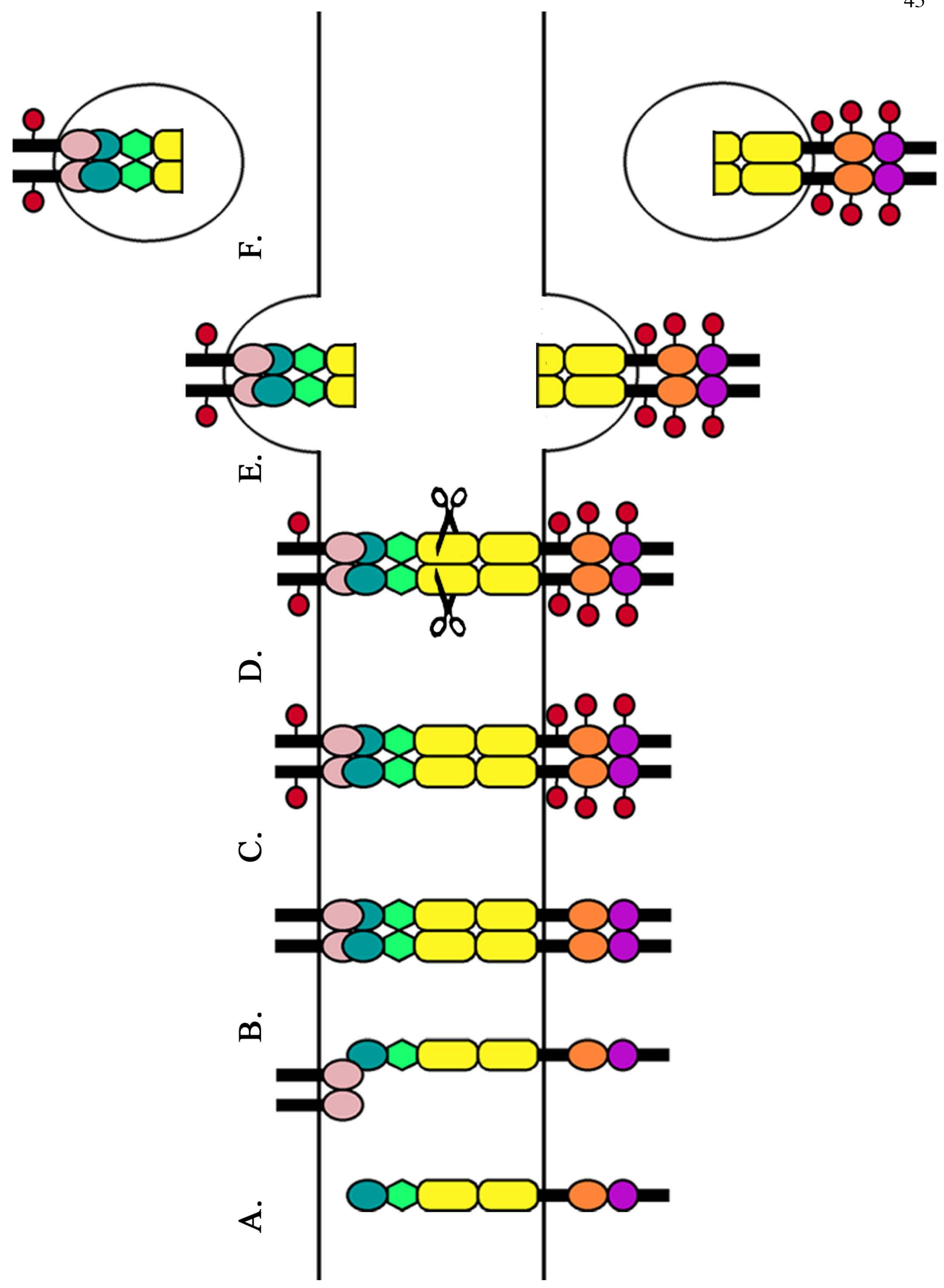


Figure 1-3. Eph/ephrin activation, proteolysis, and endocytosis.

(A) When Eph expressing cells and ephrin-B expressing cells are in close proximity, the ligand dimer binds to two separate Eph molecules through high affinity interfaces. (B) After the two Eph receptors bind to one ligand pair, they dimerize and form a tight tetramer. (C) The dimerization of Eph receptors leads to trans-phosphorylation of the tyrosine residues and activates the kinase activity. The B class ligands are also phosphorylated by RTKs or cytoplasmic kinases. Both the activated Eph receptors and ephrin ligands are fully capable of binding to downstream mediators and turning on signaling cascades. (D) One mechanism to terminate signaling involves the dissociation of the Eph-ephrin complexes by proteolysis. (E)-(F) The lysed Eph and ephrin molecules sometimes are recycled through endocytosis. 
A.

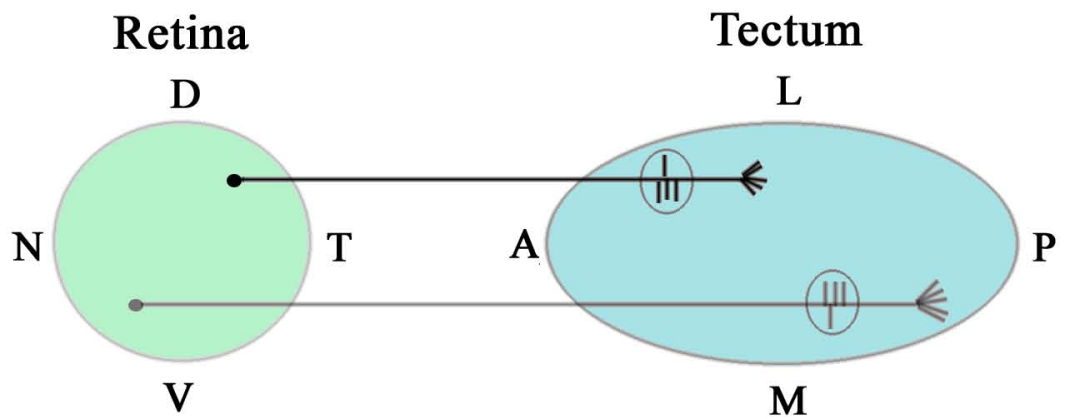

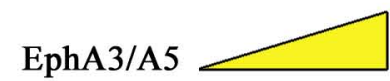

EphA4

ephrinA2/A5

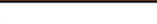

ephrinA2/A5

EphA

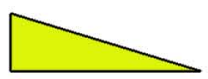

Tectum

L

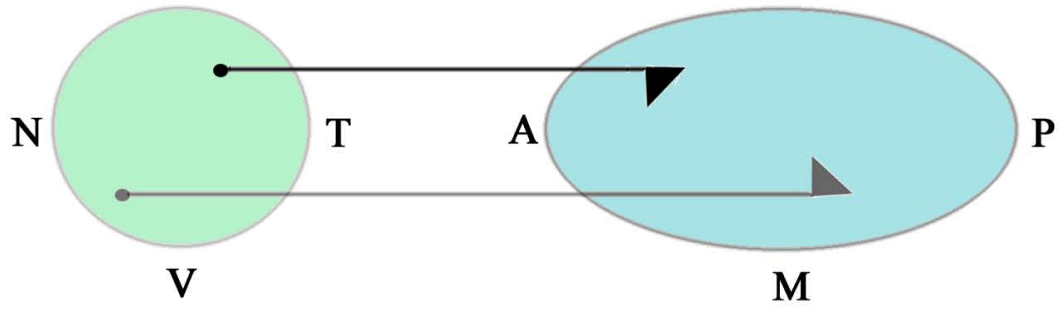


Figure 1-4. A schematic representation of the retinal-tectal projection in chick.

(A) During development, the nasal retinal neurons express a low level of EphA receptors and extend to the posterior tectum where ephrin-A expression is high. Conversely, temporal neurons expressing high levels of EphAs project to the anterior tectum where ligand expression is low. The growth cones usually travel past their termination zone (as indicated by small circles). Collateral branches form on the axon shafts within the termination zone, laterally on the nasal neurons and medially on the temporal neurons. The graded expression of Eph receptors and ephrins are indicated below the graph. (B) The topographic projection between the retina and the tectum is complete. As shown, the growth cones retract to their final destination and form synaptic connects with their targets. In addition, all ectopic branches have disappeared. 
Signaling proteins that bind to Eph receptors

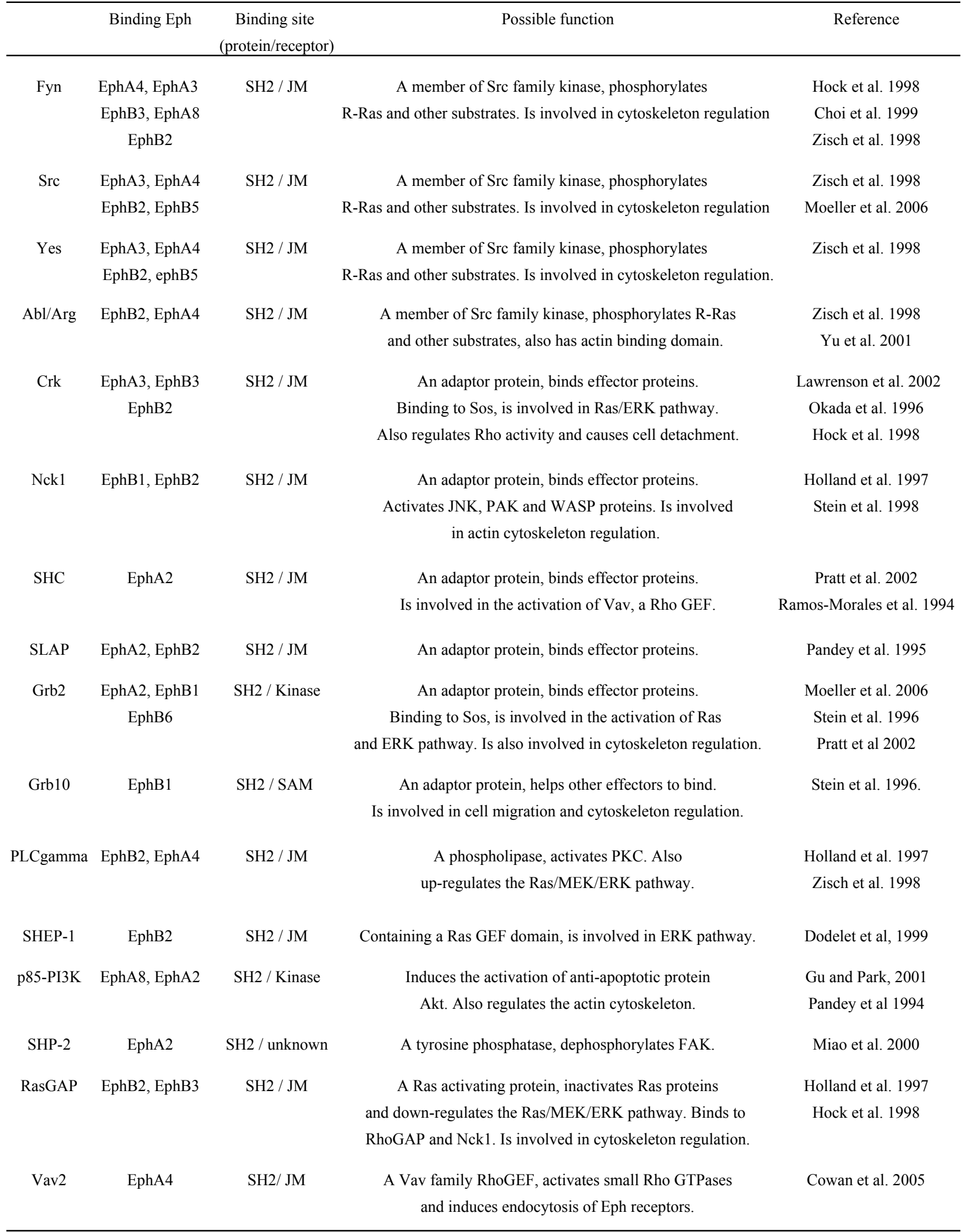


Signaling proteins that bind to Eph receptors (Continued)

\begin{tabular}{|c|c|c|c|c|}
\hline & Binding Eph & $\begin{array}{c}\text { Binding site } \\
\text { (protein/receptor) }\end{array}$ & Possible function & Reference \\
\hline PICK1 & EphB2, EphA7 & PDZ / PDZ motif & Interacts with synaptic receptors. Is involved in PKC activation. & Torres et al. 1998 \\
\hline GRIP & EphB2, EphA7 & PDZ / PDZ motif & $\begin{array}{l}\text { Interacts with synaptic receptors and serves as a scaffold } \\
\text { protein }\end{array}$ & $\begin{array}{c}\text { Torres et al. } 1998 \\
\text { Bruckner et al. } 1999\end{array}$ \\
\hline Syntenin & EphB2, EphA7 & PDZ / PDZ motif & $\begin{array}{l}\text { A syndecan binding protein, is involved in } \\
\text { actin dynamics and synaptic functions. }\end{array}$ & $\begin{array}{l}\text { Torres et al. } 1998 \\
\text { Koroll et al. } 2001\end{array}$ \\
\hline AF6 & $\begin{array}{l}\text { EphB2, EphB3 } \\
\text { EphB5, EphA7 }\end{array}$ & PDZ / PDZ motif & A Ras binding protein, recruits Ras proteins. & Hock et al. 1998 \\
\hline Tiam1 & EphA2 & PDZ / PDZ motif & $\begin{array}{l}\text { A Rac GEF, binds to ephrinB1, up-regulates Rac activity } \\
\text { and induces neurite outgrowth }\end{array}$ & Tanaka et al. 2004 \\
\hline Dishevelled & EphB2 & PDZ/ PDZ motif & $\begin{array}{c}\text { A PDZ domain containing protein, regulates signal } \\
\text { transduction in Wnt pathway. A Rho GEF, induces Rho } \\
\text { up-regulation and cell repulsion }\end{array}$ & Tanaka et al. 2003 \\
\hline Ephexin & EphA4 & DH-PH/ kinase & $\begin{array}{l}\text { A neuronal RhoGEF, preferentially activates Rho small } \\
\text { GTPases and leads to growth cone collapse }\end{array}$ & Shamah et al. 2001 \\
\hline LMW-PTP & EphB1, EphB2 & ? / SAM & A phosphotyrosine phosphatase, promotes cell adhesion & Stein et al. 1998 \\
\hline FAK & EphA2 & $? / ?$ & $\begin{array}{l}\text { Is directly involved in integrin signaling. Is phosphorylated } \\
\text { by EphA2 and dephosphorylated by SHP- } 2 \text {. }\end{array}$ & Miao et al. 2000 \\
\hline Syndecan-2 & EphB2 & $? / ?$ & $\begin{array}{l}\text { A transmembrane heparin sulfate proteoglycan, induces } \\
\text { spine formation by clustering }\end{array}$ & Ethell et al. 2001 \\
\hline NR1 & EphB2 & $? / ?$ & $\begin{array}{l}\text { NMDA type glutamate receptor, is involved in Calcium } \\
\text { influx regulation. Is phosphorylated by Eph receptors. }\end{array}$ & $\begin{array}{c}\text { Grunwald et al. } 2001 \\
\text { Takasu et al. } 2002\end{array}$ \\
\hline ADAM17 & EphB2 & $? / ?$ & $\begin{array}{l}\text { A metalloprotease in the extracellular space, cleaves } \\
\text { Eph receptors and leads to the termination of signaling. }\end{array}$ & $\begin{array}{l}\text { Mancia et al. } 2005 \\
\text { Hattori et al. } 2000\end{array}$ \\
\hline
\end{tabular}


Table 1-1. Signaling proteins that interact with Eph receptors.

Signaling proteins that bind to Eph receptors are summarized in the table. Also summarized in the table are the binding sites that mediate the interactions and the possible functions regulated by these signaling protein. 


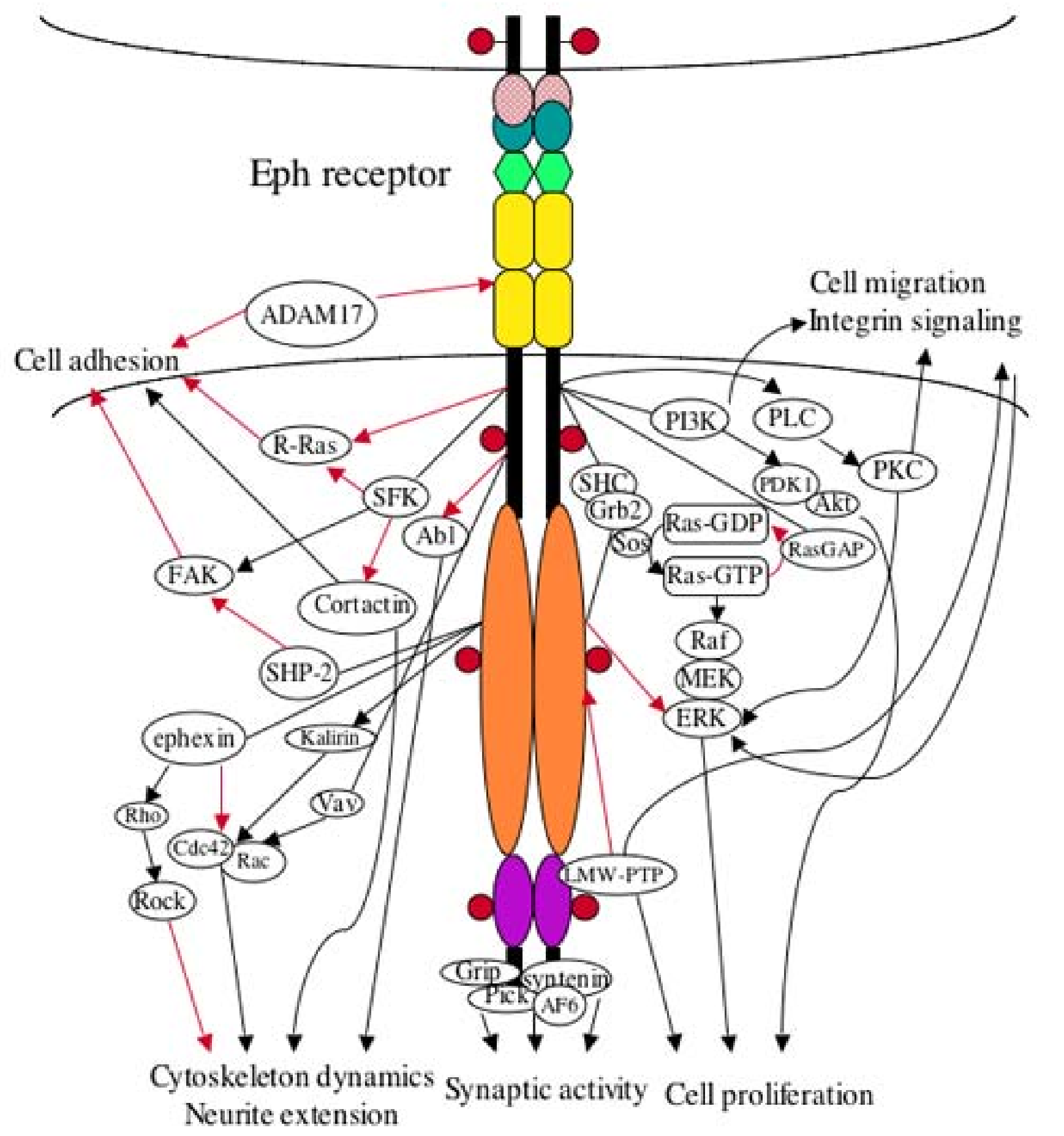


Figure 1-5. Signaling pathways implicated in Eph receptor mediated signal transduction.

Eph receptor signaling regulates cell adhesion, cell migration, cytoskeleton dynamics, cell proliferation, and synaptic activity via the many pathways that are turned on after receptor activation and phosphorylation. $\mathrm{SH} 2$ adaptor proteins including Grb2, SHC and Crk are responsible for organizing the assembly of Eph/effector protein complex. Eph receptors are able to inhibit Ras/MEK/ERK pathway, which disrupts integrin signaling and promotes cell proliferation. Ephexin and other RhoGEFs regulate the activity of small Rho GTPases and lead to cytoskeletal rearrangements. In addition, Eph receptors can dephosphorylate FAK through Src family kinases and reduce adhesion. The Cterminus of Eph receptors are capable of recruiting proteins containing PDZ binding domains, which help to localize Eph receptors to specific locations such as synapses or growth cones. In the extracellular space, ADAM proteins associate with Eph receptors and catalyze the proteolysis of the receptor/ligand pair. In this figure, the arrows show the direction in which the multi-step signaling cascade occurs. The black arrows indicate a positive effect, whereas red lines show inhibitory effects. 
Signaling proteins that bind to ephrins

\begin{tabular}{|c|c|c|c|c|}
\hline & Binding ephrin & $\begin{array}{c}\text { Binding site } \\
\text { (protein/receptor) }\end{array}$ & Possible function & Reference \\
\hline PICK1 & ephrin-B1 & PDZ / PDZ motif & Interacts with synaptic receptors. Is involved in PKC activation. & Torres et al. 1998 \\
\hline GRIP & ephrin-B1 & PDZ / PDZ motif & $\begin{array}{l}\text { Interacts with glutamate receptors. Serves as a scaffold } \\
\text { protein to localize ephrin to the synapse. }\end{array}$ & Torres et al. 1998 \\
\hline GRIP2 & ephrin-B1 & PDZ / PDZ motif & $\begin{array}{l}\text { Interacts with glutamate receptors. Servess as a scaffold } \\
\text { protein to localize ephrin to the synapse. }\end{array}$ & Lin et al. 1999 \\
\hline Syntenin & $\begin{array}{l}\text { ephrin-B1 } \\
\text { ephrin-B2 }\end{array}$ & PDZ / PDZ motif & $\begin{array}{l}\text { A syndecan binding protein, is involved in } \\
\text { actin dynamics and synaptic functions. }\end{array}$ & $\begin{array}{l}\text { Torres et al. } 1998 \\
\text { Lin et al. } 1999\end{array}$ \\
\hline PTP-BL & ephrin-B1 & PDZ / PDZ motif & $\begin{array}{l}\text { A PDZ domain containing tyrosine phosphatase, regulates } \\
\text { negatively the phosphorylation of ephrin and Src kinases. }\end{array}$ & $\begin{array}{l}\text { Palmer et al. } 2002 \\
\text { Lin et al. } 1999\end{array}$ \\
\hline PDZ-RGS3 & ephrin-B1 & PDZ / PDZ motif & $\begin{array}{l}\text { A regulator of heterotrimeric } \mathrm{G} \text { protein, regulates } \\
\text { G protein coupled chemoattraction signaling. }\end{array}$ & Lu et al. 2001, 2004 \\
\hline PHIP & ephrin-B1 & PDZ / PDZ motif & $\begin{array}{l}\text { Pleckstrin homology domain interacting protein, is } \\
\text { implicated in Insulin receptor signaling. }\end{array}$ & Lin et al, 1999 \\
\hline Tiam1 & ephrin-B1 & PDZ / PDZ motif & $\begin{array}{l}\text { A Rac GEF, binds to ephrinB1, up-regulates Rac activity } \\
\text { and induces neurite outgrowth }\end{array}$ & Tanaka et al. 2004 \\
\hline Dishevelled & $\begin{array}{l}\text { ephrin-B1 } \\
\text { ephrin-B2 }\end{array}$ & PDZ/ PDZ motif & $\begin{array}{c}\text { A PDZ domain containing protein, regulates signal } \\
\text { transduction in Wnt pathway. Also a Rho GEF, induces Rho } \\
\text { up-regulation and cell repulsion }\end{array}$ & Tanaka et al. 2003 \\
\hline Nck2 & $\begin{array}{l}\text { ephrin-B1 } \\
\text { ephrin-B2 }\end{array}$ & $\mathrm{SH} 2 / ?$ & $\begin{array}{l}\text { Dock homolog, a SH2/SH3 adaptor protein, binds to } \\
\text { effectors like Abi-1, CAP, axin, regulates cytoskeleton. }\end{array}$ & Cowan et al. 2001 \\
\hline $\mathrm{Src}$ & ephrin-B1 & $\mathrm{SH} 2 / ?$ & $\begin{array}{l}\text { A Src family kinase, phosphorylates ephrins as well } \\
\text { as other substrates. }\end{array}$ & $\begin{array}{l}\text { Palmer et al. } 2002 \\
\text { Holland et al. } 1996\end{array}$ \\
\hline Fyn & ephrin-B1 & $\mathrm{SH} 2 / ?$ & $\begin{array}{l}\text { A Src family kinase, phosphorylates ephrins as well } \\
\text { as other substrates. Is important for ephrin induced cell adhesion. }\end{array}$ & Davy et al. 1999 \\
\hline Yes & ephrin-B1 & $\mathrm{SH} 2 / ?$ & $\begin{array}{l}\text { A Src family kinase, phosphorylate ephrins as well } \\
\text { as other substrates. }\end{array}$ & Palmer et al. 2002 \\
\hline ADAM10 & ephrin-A2 & $? / ?$ & $\begin{array}{l}\text { A metalloprotease in the extracellular spac, cleaves } \\
\text { Eph receptors and leads to cell detachment and the } \\
\text { termination of signaling. }\end{array}$ & Hattori et al. 2000 \\
\hline
\end{tabular}


Table 1-2. Signaling proteins downstream of ephrin ligands.

The binding proteins of ephrin ligands are listed. Similar to Table 1-1, the information about their binding sites and their functions is also included. 


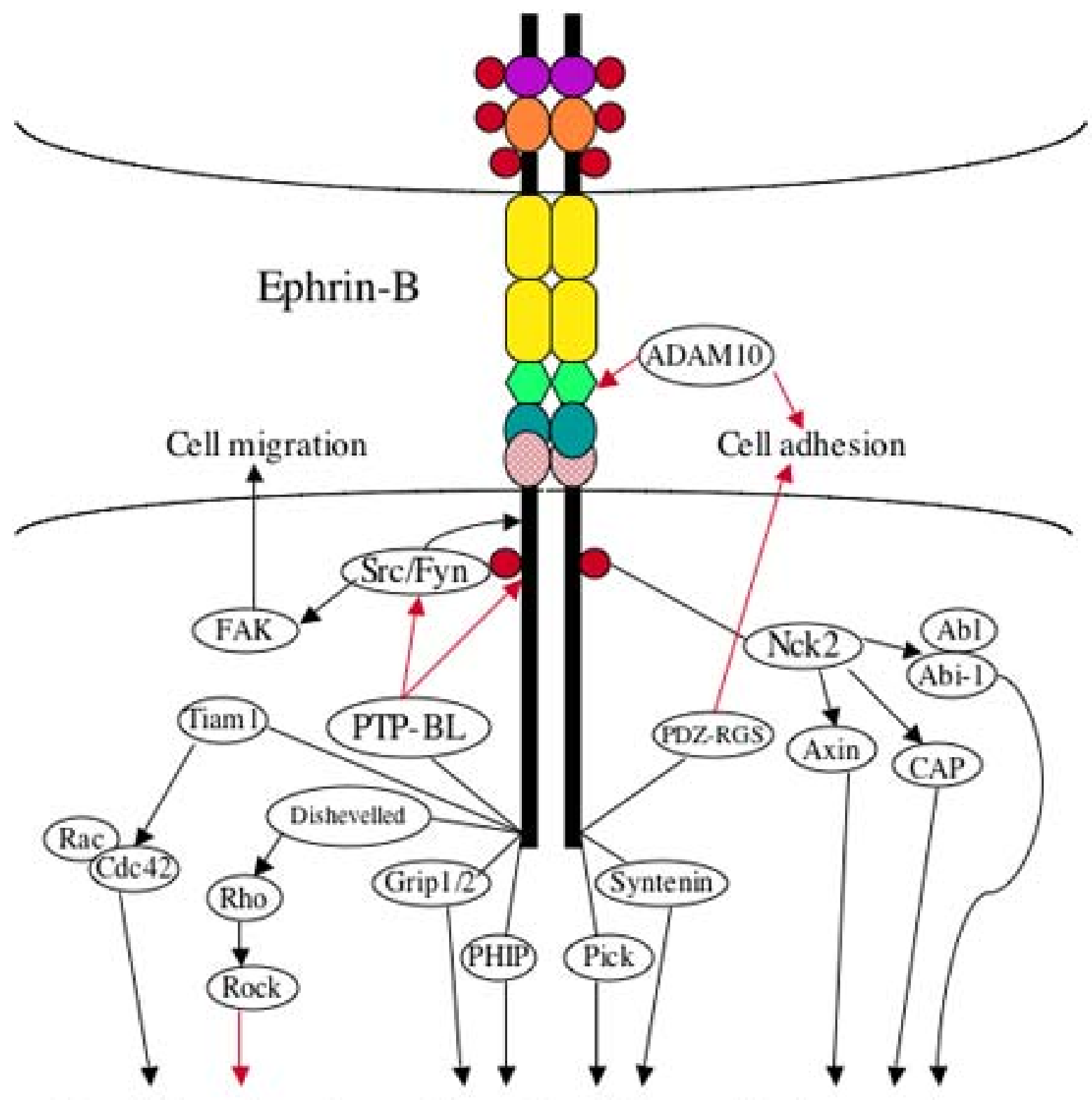

Cytoskeleton dynamics Synaptic activity Neurite extension 
Figure 1-6. Signaling pathways involved in reverse signaling through ephrin-Bs.

The aggregation of Eph receptors and their ligands potentially ephrins, especially the ephrin-Bs. Through phosphorylation by Src kinases and other RTKs, the ephrin-Bs are activated. The signaling through ephrins mediates modulation of cell migration, cell adhesion, cytoskeletal dynamics, neurite extension, and synaptic activity. Src family kinases regulate cell adhesion directly through phosphorylating FAK. Nck2, bound to activated ephrins, regulates cytoskeletal dynamics through Axin, CAP and Abi-1. Similar to Eph receptors, ephrins also recruit a great number of PDZ domain proteins. Among them, Tiam1 and Dishevelled modulate neurite outgrowth and growth cone collapse by affecting the balance of small Rho GTPases. PTP-BL antagonizes the activity of cytoplasmic kinases and dephosphorylates ephrins. Other PDZ domain proteins mediate the interaction between ephrins and receptors, especially at the synapses. Similar to Figure. 1-5, the black arrows mark positive effects and red arrows are indicative of inhibitory effects. 


\section{Chapter II. Screening for EphA receptor binding proteins}

\section{using the yeast two-hybrid method}

\section{Introduction}

The yeast two-hybrid screen technique has been established as a powerful method to identify protein-protein interactions. Originally, this method made use of the yeast transcriptional activator GAL4 protein (Keegan et al. 1986; Fields and Song 1989). GAL4 protein regulates the transcription of genes involved in galactose metabolism. It consists of a N-terminal DNA binding domain and a C-terminal transcriptional activation domain. These two domains are functionally separate, but are both required for GAL4 protein to induce transcription activation (Brent and Ptashne 1985). In the initial experimental design, two fusion proteins were generated: DNA binding domain fused to protein $\mathrm{X}$ and activation domain fused to protein $\mathrm{Y}$. These fusion proteins were coexpressed in yeast cells that also carried a reporter plasmid [(Chien et al. 1991), Fig. 2-1 A]. The association of protein $\mathrm{X}$ and protein $\mathrm{Y}$ brings the GAL4 DNA binding domain and GAL4 activation domain together, and leads to the expression of the reporter gene LacZ. As a result, the interaction of $\mathrm{X}-\mathrm{Y}$ is detected by examining the LacZ activity in these yeast cells.

The yeast two-hybrid method we used is a modified system based on similar principles. Instead of GAL4 DNA binding domain, the E. coli LexA protein is fused to protein X (usually the molecule of interest), while the acid blob domain B42 replaces the GAL4 activation domain in the Y fusion protein (contained in a cDNA expression library). When the LexA protein and B42 domain are brought together through direct X- 
Y interaction, the protein complex associates with the UAS and turns on LacZ gene transcription. Therefore, by testing for LacZ gene activity, we are able to screen for strong binding proteins in the cDNA library on large scale with confidence (Fig. 2-1 B).

Compared to the original yeast two-hybrid system, the modified method has several advantages. First, since yeast strains and reporter vectors of varying sensitivity are available, we are able to tailor the screen to suit our needs and select only for the strongest binding proteins. Second, the bait plasmid contains a nuclear localization sequence (NLS), which ensures that the bait fusion protein enters the nucleus and binds to the UAS. Thirdly, LexA proteins dimerize constitutively, allowing activation of the Eph receptor kinase domain and leading to phosphorylation on key tyrosine residues. In addition, since prokaryotic proteins are used in an eukaryotic system, the protein-protein interactions we identify through this system are highly specific and the occurrence of false positives is greatly reduced. Lastly, several tests are incorporated into the experimental design to confirm the validity of the binding proteins isolated in the preliminary screen.

\section{Results}

\section{Construction of the bait plasmid and screening of a mouse brain cDNA library}

A flow chart shows the overall procedure of the yeast two-hybrid (Fig. 2-2). The cytoplasmic domain of EphA5 (amino acid 564-1016) was first cloned and inserted into the bait plasmid pEG202-NLS behind LexA sequence. This plasmid has a nuclear localization sequence and ensures the nuclear delivery of the expressed bait protein. With LexA on the N-terminal, the complete bait (LexA-EphA5intra) contains the 
juxtamembrane domain, the tyrosine kinase domain, and the SAM domain of EphA5 receptor (Fig. 2-3 A).

The bait plasmid pEG202-NLS-EphA5intra was first transformed into strain EGY188 yeast cells, in which the preliminary screen was performed. EGY188 strain was chosen because of its low sensitivity, which ensured that only strong interactors would be isolated. The inserted gene was confirmed to be in the correct reading frame by Western blot with anti-EphA5 antibody and anti-lexA antibody. The size of the bait fusion protein was estimated to be about $72 \mathrm{KD}$ (Fig. 2-3 B). Furthermore, it was confirmed that the resulted bait protein was delivered into the nucleus but was unable to turn on the reporter gene transcription on its own (data not shown). Later, EGY188 cells carrying pEG202NLS-EphA5intra were co-transformed with pJK103, a reporter plasmid of medium sensitivity and a mouse embryonic cDNA expression library cloned in target plasmid pJG4-5.

The transformants carrying all three plasmids were diluted and plated on culture medium plates until individual colonies grew. The colonies were replicated onto a pair of culture plates, which contained glucose or galactose, respectively. Two days later, the colonies on the plates were lifted off onto nitrocellulose filter paper and the following test for LacZ expression was conducted. The colonies on filter papers were frozen and thawed repeatedly to release cell contents. Then the filters were submerged in a solution containing $\mathrm{X}-\mathrm{Gal}$ for two hours to allow the LacZ reaction to take place. The design of the system dictated that a colony was positive only if it had LacZ expression on the plate containing galactose but not on the other plate. Since LacZ converts X-Gal to a blue 
precipitate, we identified all positive colonies by means of comparing the intensity of the blue color on two plates (Fig. 2-4).

Plasmid DNA was then extracted from the positive colonies and sequenced. The identities of these genes were searched in public databases through BLAST program (http://www.ncbi.nlm.nih.gov/BLAST). In order to confirm the validity of the preliminary screen, we tested the positive cDNA sequences through individual mating tests. The extracted target plasmid and the reporter plasmid were co-transformed into EGY188 yeast strain, while the bait plasmid pEG202-NLS-EphA5intra, an empty bait plasmid pBait or a negative control plasmid pRHFM were each transformed into EGY40 strain. To conduct the mating test (Fig. 2-5 A), EGY188 carrying both the positive target plasmid and the reporter plasmid was streaked first across the culture plate horizontally. Then, the transformed EGY40 were streaked perpendicularly across the first streak. As a result, the two parental strains mixed and produced offspring cells, which inherited all three plasmids from the parental cells. When LacZ filter assay was performed on these

offspring cells, proteins with real binding with EphA5 turned on the LacZ reaction in the presence of EphA5 bait expression but not the control proteins (Fig. 2-5 B). By contrast, false positives were unable to catalyze the conversion of X-gal in any of the three streaks (Fig. 2-5 B).

\section{Fifteen interactors were identified through the screen}

In our study, a total of $10^{7}$ clones were screened and about 300 clones were sequenced. Despite the power of yeast two-hybrid, it has been shown in the past that some clones isolated through this method are mere artifacts and they appear in screens 
regardless of the bait used. Luckily, Dr. Erica Golemis and Dr. Ilya Serbriiskii of the Fox Chase Cancer Center consolidated data from more than 100 yeast two-hybrid screens and posted the false positives on their website (http://www.fccc.edu/research/labs/golemis/ Interaction TrapInWork.html ). These proteins include heat shock proteins, ribosomal proteins, ubiquitin, proteasome subunits, mitochondrial proteins, transcription elongation factors and so on. Using this information, we were able to eliminate common false positive genes from our results. A large portion of the remaining positive genes was unknown cDNA sequences, which had been documented but never identified.

Consequently, we were not able to analyze them further at that time. After individual mating tests were performed on the rest of the positive clones, fifteen genes tested positive and were considered real positives. These proteins were studied through literature search and their possible roles in Eph receptor signaling were evaluated. Importantly, many of these positive genes were encountered multiple times in our screen, indicating the consistency of the method.

\section{Nck1 binds strongly with EphA5 receptor}

Among the 15 binding proteins, one protein with the strongest binding with EphA5 is Nck1, a SH2/SH3 domain adaptor protein. The cDNA fragment of Nck1 isolated in the screen, when compared to full length Nck1 sequence, consisted of the SH2 domain, the second and third SH3 domains (Fig. 2-6 A). As shown through the mating test, this cDNA product binds to the bait LexA-EphA5intra and turns on LacZ gene transcription (Fig. 2-6 B). The association between Nck1 and EphA5 was highly specific since no 
LacZ activity was detected when either control plasmid was used in place of the bait plasmid.

Intriguingly, when a similar yeast two-hybrid screen was conducted using EphA3 intracellular domain as the bait in our lab, Nck1 was also one strong interactor (Dr. Guanfang Shi, unpublished data). Therefore, we hypothesize that the adaptor protein Nck1 binds to multiple EphA receptors and play critical roles in their signaling.

\section{Discussion}

\section{The identified EphA interactors regulate different aspects of signal transduction}

Fifteen binding interactors of EphA5 were identified in the yeast two-hybrid screen and they are summarized in Table 2-1. According to their known functions, they are grouped into 4 categories (1) membrane trafficking related genes, (2) cytoskeleton regulation related genes, (3) tumor regulation related genes and (4) enzymes.

Five of the identified binding proteins are implicated in the regulation of membrane trafficking. They are Arl3 (ADP-ribosylation-like 3), CDCrel-1 (cell division control related protein-1), OSBP (oxysterol-binding protein), Rab2 (Ras-like small GTPase Rab2) and Rab11 (Ras-like small GTPase Rab11) (Beites et al. 1999; Cheung et al. 2002; Kahn et al. 2005; Savina et al. 2005; Olkkonen et al. 2006; Perry and Ridgway 2006). This group of proteins is possibly important in the recycling of the Eph receptors, which determines the duration of Eph signaling. For example, Rab2 and Rab11 are both members of Ras-like small GTPase Rab family. They have been recognized as key mediators during endocytosis and the transport of the resulting vesicles in cell cytosol (Stein et al. 2003; Ceresa 2006). 
An important event during EphA receptor signaling is cytoskeletal restructuring (Bruckner and Klein 1998; Murai and Pasquale 2005; Sahin et al. 2005). Usually, upon EphA activation, activated effector proteins modulate cytoskeleton dynamics. These modulations lead to cellular events such as cell migration or process retraction. Therefore, it was unsurprising to find several positive clones encoded for proteins involved in cytoskeleton dynamics. These proteins include Arl3 (ADP-ribosylation-like 3 protein), cdc42 (small Rho GTPase), Mayven, Mpp3 (MAGUK family member 3) and Nck1 (non-catalytic region of tyrosine kinase adaptor protein 1) (Bladt et al. 2003; Sahin et al. 2005; Williams et al. 2005; Kantardzhieva et al. 2006; Zhou et al. 2006).

Another group of interacting proteins include Lgil (Leucine-rich, glioma inactivated 1), Mayven, NOV (Nephroblastoma overexpressed gene), PELOTA, SPARC (secreted acidic cysterine rich glycoprotein) and TCTP (translationally-controlled tumor protein) (Jendraschak and Sage 1996; Kunapuli et al. 2004; Kyurkchiev et al. 2004; Tuynder et al. 2004; Bu et al. 2005; Xi et al. 2005). All proteins in this group play roles in tumorigenesis. Since Eph receptors are also implicated in tumor progression (Nakamoto and Bergemann 2002; Surawska et al. 2004), the identification of these proteins reveals possible pathways downstream of Eph receptors in tumor development. Furthermore, the disruption of these pathways may be effective in treating cancer.

In our study, we also found that non-receptor tyrosine phosphatase Ptpn5 (Barr and Knapp 2006) bound to the cytoplasmic domain of EphA5. Previously, it has been reported that protein tyrosine phosphatase Shp2 (Miao et al. 2000), low molecular weight PTPs (LMW-PTPs) (Kikawa et al. 2002; Parri et al. 2005) and the receptor-type phosphatase PTPO (Shintani et al. 2006) associate with Eph receptors and modulate the 
phosphorylation level of substrates. Therefore, the identification of Ptpn5 indicates that Ptpn5 may play a similar role.

The results we obtained are probably far from being comprehensive. Proteins found to bind to EphA5 in the past, for example, were not identified in this screen, indicating that the downstream signaling of EphA receptors is complex and there are likely to be more unidentified interactors. Therefore, it would be rewarding to continue the yeast twohybrid screen and isolate more binding proteins.

\section{The binding of Nck1 and EphA receptors links activated EphA receptor and the cytoskeleton regulation.}

Nck1 (also known as Nck, Nck $\alpha$ ) stands for non-catalytic region of tyrosine kinase (McCarty 1998). Nck1 is a SH2/SH3 domain-containing adaptor protein (Buday et al. 2002; Rivera et al. 2006). SH2/SH3 adaptor proteins consist of various numbers of SH2 and SH3 domains. They lack have intrinsic enzymatic activities, but function as scaffold proteins. SH2/SH3 daptor proteins bind to phosphorylated tyrosines on receptor tyrosine kinases through SH2 domains. Through SH3 domains, they bind to proline-rich domains of effector proteins and bring them in the proximity of the phosphorylated receptors (Birge et al. 1996; Mayer and Gupta 1998; McCarty 1998). Other than Nck1, SH2/SH3 containing adaptor proteins include Nck2, Grb2 and Crk (Pawson 1994; McCarty 1998; Buday et al. 2002).

In mammalian cells, there are two members in Nck family, Nck1 and Nck2 (a.k.a. $\mathrm{Nck} \beta$ ). They both possess three $\mathrm{SH} 3$ domains in the $\mathrm{N}$-terminus and one $\mathrm{SH} 2$ domain in C-terminus. Nck1 and Nck2 share high homology (60\%) with each other, especially in 
the functional domains. Nck family members bind to receptor tyrosine kinases such as EGFR, PDGFR and VEGFR and receptors such as DCC and Robo (Roche et al. 1996; Tang et al. 1997; Li et al. 2002; Stoletov et al. 2004). It has been shown that Nck1 plays a role in the signaling of both EphB1 and EphB2 (Holland et al. 1997; Stein et al. 1998). However, the association of Nck1 with EphA receptors has never been established before.

So far, 30 effectors have been identified for Nck1 and interestingly, many of these proteins were found to be associated with cytoskeleton regulation (McCarty 1998; Buday et al. 2002). For example, WASP family members and PAK proteins (Zhao et al. 2000; Rohatgi et al. 2001; Zalevsky et al. 2001) have been both implicated in actin restructuring. Therefore, our identification of Nck1 in the screen suggests that the function of Nck1 may be involved in the signaling of some EphA receptors by linking active receptors with the cytoskeletal machinery. 
A.

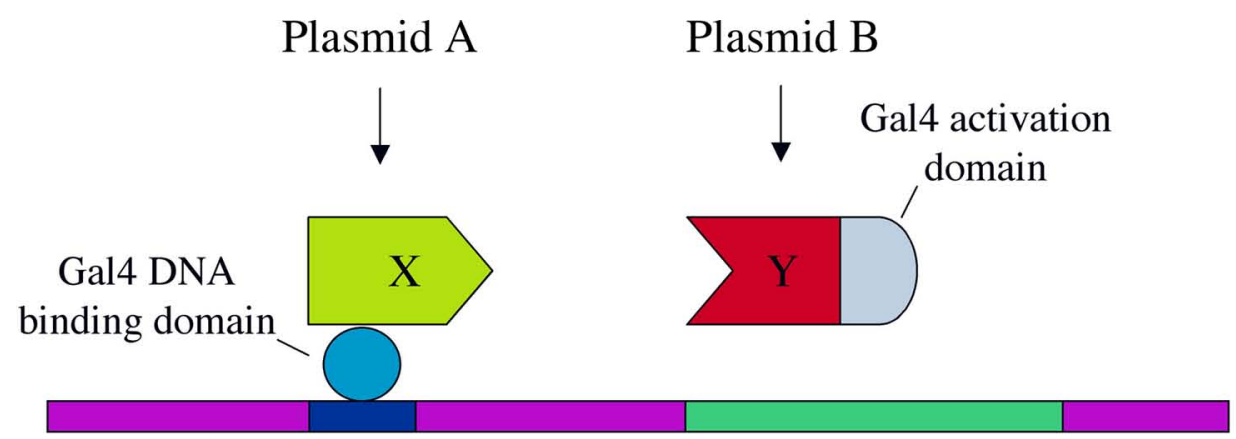

Reporter plasmid

$$
\mathrm{UAS}_{\mathrm{G}} \quad \text { GAL-lacZ }
$$

B.

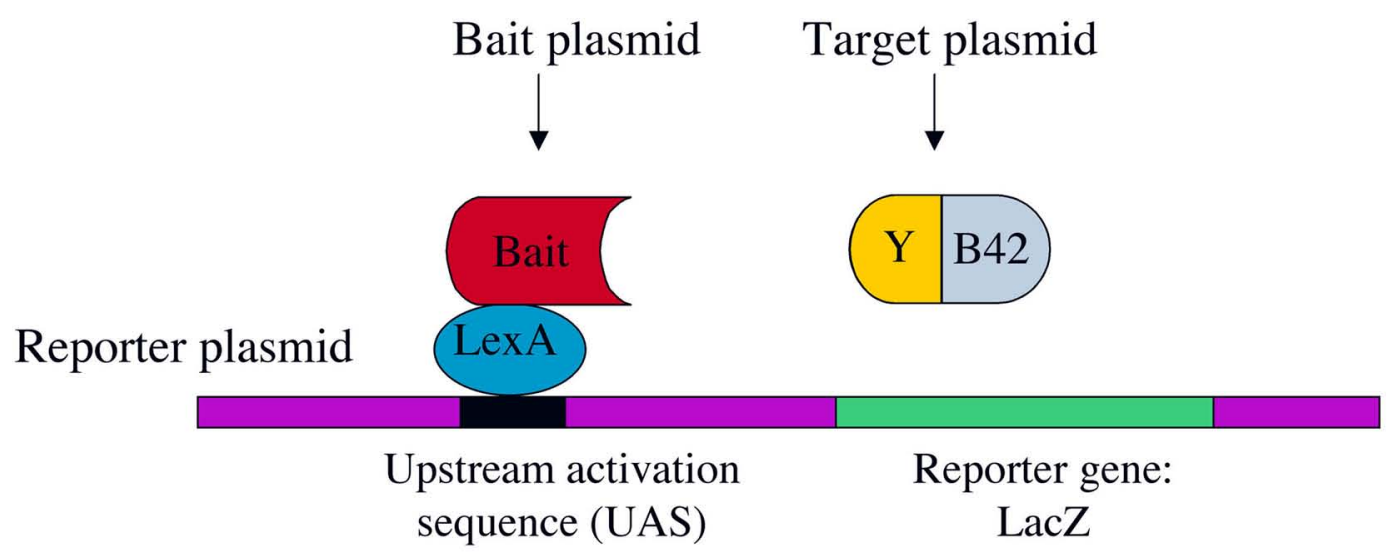


Figure 2-1. A schematic representation of the yeast two-hybrid screen.

(A) The original experimental design. Protein $\mathrm{X}$ and $\mathrm{Y}$ are fused to a Gal4 DNA binding domain and activation domain respectively. Strong binding between protein $\mathrm{X}$ and $\mathrm{Y}$ brings the two functional domains of Gal4 together, leading to transcription and expression of the reporter gene. (B) The current DupLEX system. This system employs a similar design in which protein $\mathrm{X}$ and $\mathrm{Y}$ are fused to $E$. coli proteins LexA protein and B42 protein instead. 


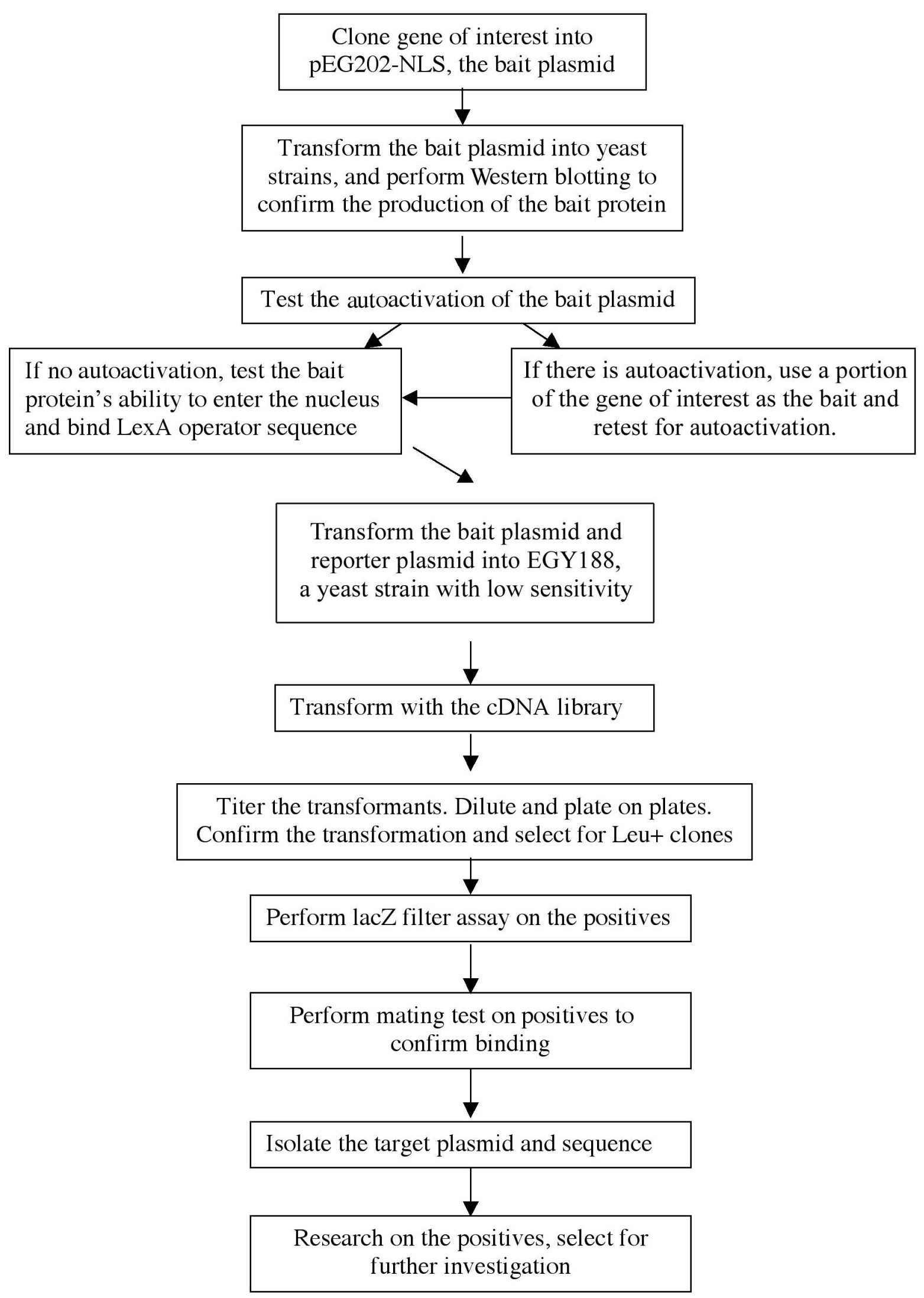


Figure 2-2. A summary of the yeast two-hybrid screen procedure.

First, the bait plasmid pEG202-NLS-EphA5intra was constructed and tested. Using this bait, a preliminary screen was performed in EGY188 and about $10^{7}$ clones from an embryonic mouse brain cDNA library were screened. Three hundred positives from the preliminary screen were then sequenced and identified. The positive clones were further confirmed through mating tests. 
A.

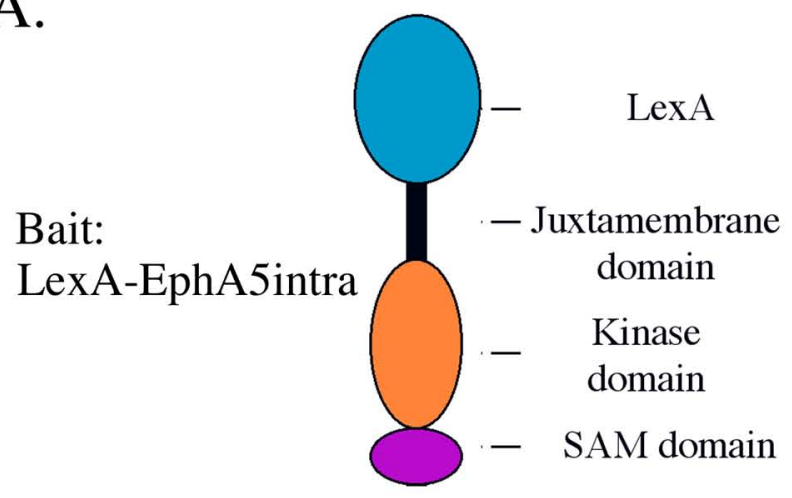

B.

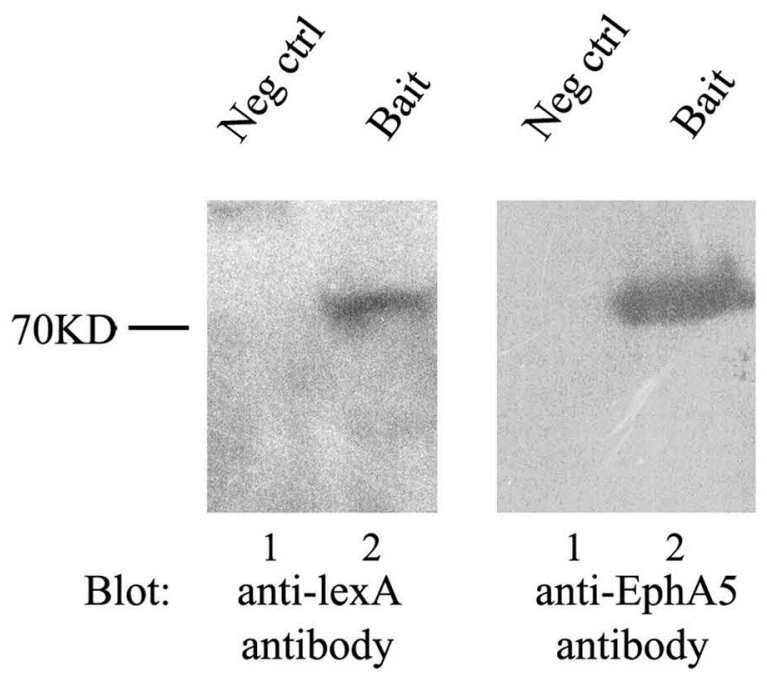


Figure 2-3. Structure and expression of the bait LexA-EphA5intra.

(A) The structure of the expressed bait protein. LexA fragment is fused to the N-terminal of the EphA5 intracellular fragment. (B) By Western blot, the expression of the bait in transformed EGY188 strain was confirmed using both anti-lexA antibody and antiEphA5 antibody. 


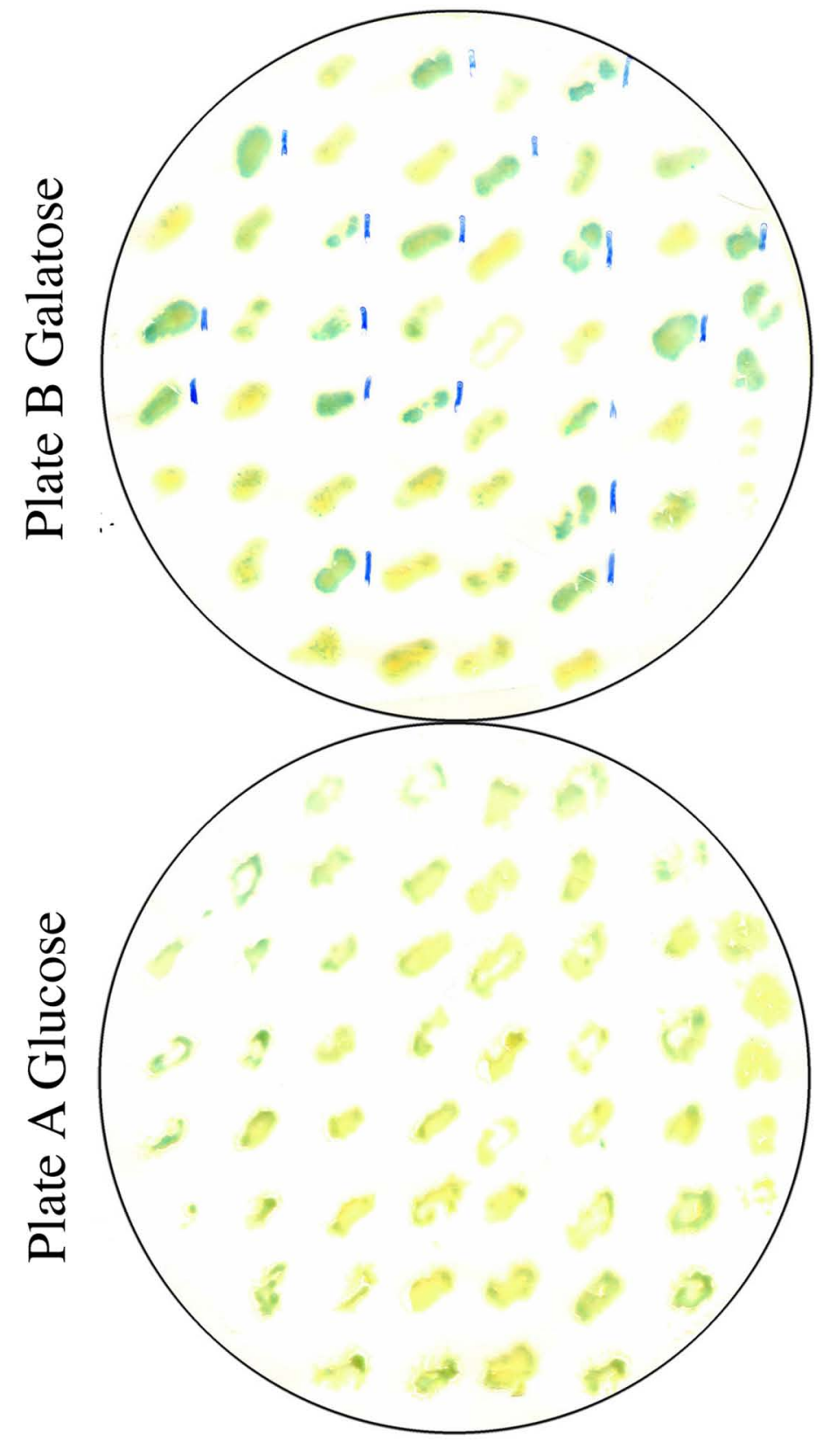


Figure 2-4. Large-scale preliminary screens for EphA5 bait through LacZ assay.

Two otherwise identical plates containing either glucose or galactose were used to culture the transformant colonies. Only colonies with LacZ expression on the galactose plate but not on the glucose plate are identified as initial positive clones, as marked by blue underline. 
A.

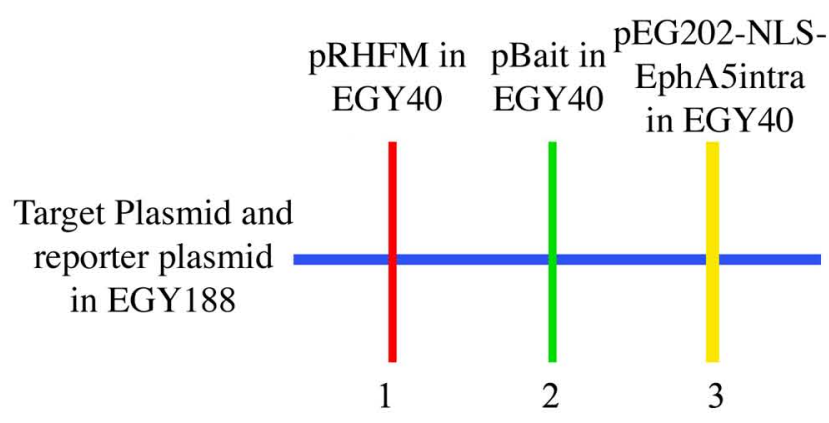

B.

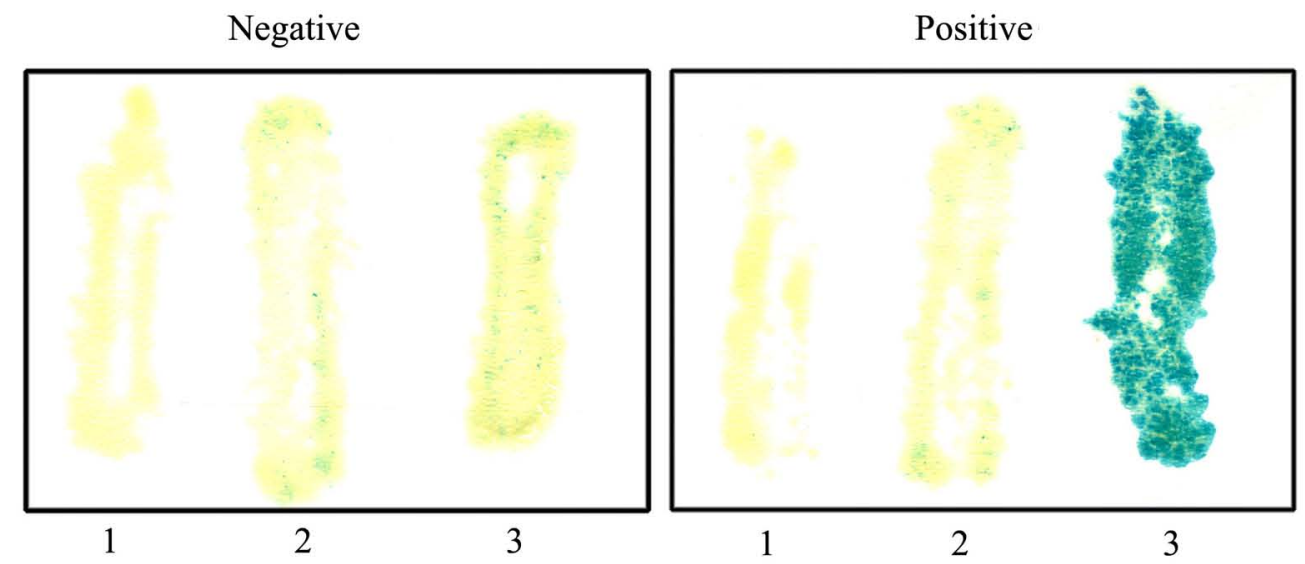


Figure 2-5. Yeast mating tests of the binding proteins identified in earlier screens. (A) A schematic representation of the mating test. Individual strains of transformed yeast cells are inoculated in the described order and mixed well. (B) Representative negative and positive test results. 
A.

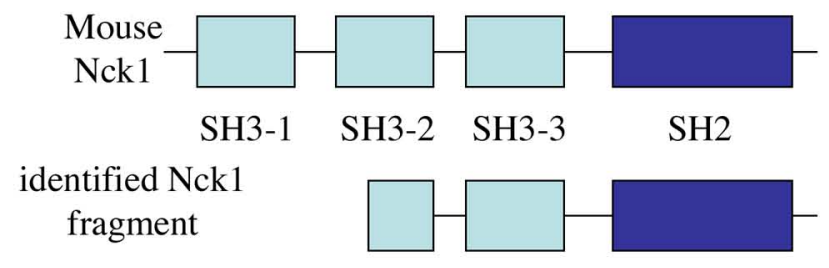

B.

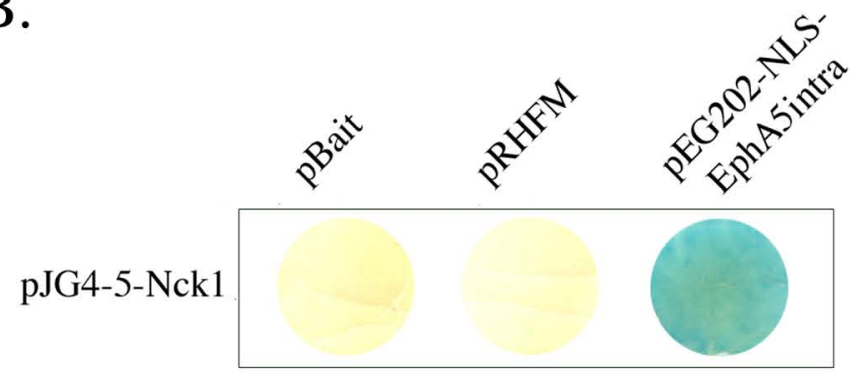


Figure 2-6. Nck1, a strong binding protein of EphA5.

(A) The Nck1 cDNA fragment isolated from the yeast two-hybrid screen, as compared to the full-length mouse Nck1 gene. (B) Positive results for Nck1 in the yeast mating test. 
Membrane trafficking

\begin{tabular}{|l|l|l|}
\hline Arl3 & ADP-ribosylation-like 3, ARF like GTPase & $\begin{array}{l}\text { Involved in vesicular transport and cytoskeleton assembly. Important to } \\
\text { photoreceptor cell development. }\end{array}$ \\
\hline $\begin{array}{l}\text { CDCrel- } \\
1\end{array}$ & $\begin{array}{l}\text { cell division control related protein-1, septin } \\
\text { family member }\end{array}$ & $\begin{array}{l}\text { Degraded by Parkin, a gene linked to juvenile Parkinsonism. Regulates } \\
\text { exocytosis, highly expressed in presynaptic nerve terminals. }\end{array}$ \\
\hline OSBP & oxysterol-binding protein & $\begin{array}{l}\text { Binds to Rab7, involved in lipid metabolism and membrane trafficking. } \\
\text { Also involved in ERK1/2 activation. }\end{array}$ \\
\hline Rab11 & Ras-like small GTPase RAB11 & Members of Rab family. Regulate vesicular trafficking. \\
\hline Rab2 & Ras-like small GTPase RAB2 & \\
\hline
\end{tabular}

\section{Cytoskeleton regulation}

\begin{tabular}{|l|l|l|}
\hline Arl3 & ADP-ribosylation-like 3, ARF like GTPase & $\begin{array}{l}\text { Involved in vesicular transport and cytoskeleton assembly. Important to } \\
\text { photoreceptor cell development. }\end{array}$ \\
\hline cdc42 & Cell division cycle 42 & $\begin{array}{l}\text { Small GTPase involved in cytoskeleton modulation and cell behavior } \\
\text { regulation. }\end{array}$ \\
\hline Mayven & Kelch like protein 2 & $\begin{array}{l}\text { Overexpressed in breast cancer and involved in oligodendrocyte process } \\
\text { formation. Binds to actin. }\end{array}$ \\
\hline Mpp3 & $\begin{array}{l}\text { membrane protein, palmitoylated 3, MAGUK } \\
\text { family member }\end{array}$ & $\begin{array}{l}\text { Contains a SH3 domain. Involved in cytoskeleton regulation and signal } \\
\text { transduction. Localized in the retinal outer membrane. }\end{array}$ \\
\hline Nck1 & $\begin{array}{l}\text { non-catalytic region of tyrosine kinase adaptor } \\
\text { protein 1 }\end{array}$ & Found to bind EGFR, PDGFR, DCC, Robo. A scaffold protein. \\
\hline
\end{tabular}

\section{Tumor regulation}

\begin{tabular}{|l|l|l|}
\hline Lgil & Leucine-rich, glioma inactivated 1 & $\begin{array}{l}\text { A secreted protein. Involved in neuronal cell survival and tumorigensis } \\
\text { suppression. }\end{array}$ \\
\hline Mayven & Kelch like protein 2 & $\begin{array}{l}\text { Overexpressed in breast cancer and involved in oligodendrocyte process } \\
\text { formation. Binds to actin. }\end{array}$ \\
\hline NOV & $\begin{array}{l}\text { nephroblastoma overexpressed gene, CCN3, a } \\
\text { CCN family member }\end{array}$ & $\begin{array}{l}\text { Involved in cancer progression. Interacts with integrins, Notch1 and other } \\
\text { receptors and ligands. }\end{array}$ \\
\hline $\begin{array}{l}\text { PELOT } \\
\text { A }\end{array}$ & & $\begin{array}{l}\text { A nuclear protein involved in cell cycle regulation. Also necessary to eye } \\
\text { patterning in Drosophila }\end{array}$ \\
\hline SPARC & $\begin{array}{l}\text { secreted acidic cysterine rich glycoprotein, } \\
\text { osteonectin }\end{array}$ & $\begin{array}{l}\text { A secreted protein involved in ECM regulation. Also exists in the } \\
\text { cytoplasm. Involved in tumor growth. Important for lens epithelial cell } \\
\text { function. }\end{array}$ \\
\hline TCTP & translationally-controlled tumor protein & $\begin{array}{l}\text { Regulates cell proliferation and cell survival, involved in tumor } \\
\text { progression }\end{array}$ \\
\hline
\end{tabular}

\section{Enzymes}

\begin{tabular}{|l|l|l|}
\hline Mpp3 & $\begin{array}{l}\text { membrane protein, palmitoylated 3, MAGUK } \\
\text { family member }\end{array}$ & $\begin{array}{l}\text { Contains a SH3 domain. Involved in cytoskeleton regulation and signal } \\
\text { transduction. Localized in the retinal outer membrane. }\end{array}$ \\
\hline Ptpn5 & $\begin{array}{l}\text { protein tyrosine phosphatase, non-receptor } \\
\text { type 5, STEP }\end{array}$ & $\begin{array}{l}\text { Inactivates MAPK and enriched in striatum. A regulator of NMDA } \\
\text { receptors. }\end{array}$ \\
\hline
\end{tabular}


Table 2-1. A summary of fifteen identified binding proteins of EphA5 in the yeast two-hybrid screen.

The positive proteins are categorized into four groups based on their functions. Their individual names, full description of their functions are listed. 


\section{Chapter III. Characterization of EphA3-Nck1 binding and evaluation of Nck1 in EphA3 signaling}

\section{Introduction}

The strong binding observed between Nck1 and EphA receptors (EphA3 and EphA5) in the yeast two-hybrid screens indicates that Nck1 may be pivotal to EphA receptor function. Thus, we set out to investigate the roles of Nck1 in the signal transduction of EphA receptor. We chose to focus on EphA3 because this particular receptor has been implicated in important functions such as topographic map formation (Ciossek et al. 1998; Otal et al. 2006). In addition, the availability of specific anti-EphA3 antibodies and EphA3 tyrosine mutants would allow the mapping of the Nck1 interaction.

To validate the binding between Nck1 and EphA3, a series of GST fusion protein pulldown experiments using fusion proteins containing full length or parts of mouse Nck1 protein were conducted. Furthermore, multiple single tyrosine mutants of EphA3 were used to map the domains through which the interaction occurs.

To demonstrate the interaction of EphA3 and Nck1, HEK293A cells transfected with both genes were used. Immunostaining showed that EphA3 receptor co-localized with Nck1 after ephrin-A5 treatment. In addition, the EphA3-Nck1 interaction was confirmed by co-immunoprecipitation.

Since EphA3 binds Nck1 strongly, we hypothesize that Nck1 is a major effector downstream of activated EphA3. This hypothesis was evaluated by assaying for cell process retraction and cell migration. In order to determine if cells with high expression 
of EphA3 responded to ligand stimulation in a Nck1 dependent way, Nck1 dominant negative proteins were introduced into EphA expressing cells.

\section{Results}

Interaction between Nck1 and EphA3 is mediated by the binding of Nck1 SH2 domain to the tyrosine 602 of EphA3.

Glutathione S-Transferase (GST) fusion proteins are commonly used in pulldown assays to test the physical interactions of two proteins (Li et al. 2002). A protein of interest, protein A is fused to GST fragment and overexpressed in bacteria in large quantity. After purification, the fusion protein is conjugated to Glutathione sepharose beads. Beads conjugated with desired fusion proteins are then incubated with cell lysates containing a high level of protein B. If proteins A and B exhibit strong binding, protein B will be detected in the precipitates with fusion protein GST-A through Western blot.

To map the binding site of Nck1, wild type EphA3 (EphA3WT) was transfected into HEK293A cells, and forty-eight hours after the transfection, the cells were stimulated with cross-linked ephrin-A5 for 30 minutes. The lysates of the treated cells were incubated with GST fusion proteins that contained either full length (GST-Nck1), the SH2 domain (GST-Nck1SH2) or the SH3 domains (GST-Nck1SH3) of mouse Nck1 (provided by Dr. L. Larose, Fig. 3-1 A). Both GST-Nck1 and GST-Nck1SH2 proteins precipitated EphA3 while GST control and GST-Nck1SH3 fusion protein did not (Fig. 31 B). An experiment performed with cell lysates of unstimulated EphA3 expressing 293A cells showed similar results except that the amount of precipitated EphA3 by GST-Nck1 and GST-Nck1SH2 was reduced (data not shown). This was probably because of the 
auto-activation of EphA3 receptors in the cell lysates due to over-expression. The pulldown studies indicate that the EphA3-Nck1 binding we identified in the yeast twohybrid screen is specific. Additionally, the SH2 domain in Nck1 is found to be responsible for EphA3 binding, since GST-Nck1SH2 precipitates EphA3 protein while GST-Nck1SH3 fails to do so (Fig. 3-1 B, lane 3 and 4). This discovery is consistent with our observation that the extracted Nck1 cDNA fragment in the yeast two-hybrid screen contains the complete SH2 domain. Indeed, when the SH2 domain was deleted from the cDNA fragment, binding with EphA no longer tested positive in the yeast mating test (data not shown).

Interestingly, Western blot analysis also shows that an unknown protein of about 100 KD was pulled down by both GST-Nck1SH3 and GST-Nck1 protein (Fig. 3-1 B, lane 4 and 5). The absence of this protein in lane 3 indicates that it was not a degradation product of EphA3, suggesting that its interaction with the anti-EphA3 antibody resulted from non-specific binding. Previous studies on Nck1 function suggest that this protein may be dynamin, a protein of about $100 \mathrm{KD}$. Dynamin is involved in endocytosis regulation and binds to Nck1 SH3 domains (Wunderlich et al. 1999; Cowan and Henkemeyer 2001).

The SH2 domains typically bind to phosphorylated tyrosine residues (Tanaka et al. 1995; Wunderlich et al. 1999; Frese et al. 2006). An unbiased screen against a degenerate phosphopeptide library further reveals that $\mathrm{SH} 2$ domain binding largely depends on the three amino acids behind the phosphotyrosine residue. For example, Nck1 SH2 domain binds preferentially to "pTyr-hydrophilic-hydrophilic-Pro/Ile" sequence (Songyang et al. 1993; Zvelebil et al. 1995). 
To identify the tyrosine residues on EphA3 to which Nck1 SH2 domain binds, several important cytoplasmic domain tyrosine residues of EphA3 known to be phosphorylated upon activation (Y596, Y602, Y736, Y779, Y937) (G Shi and R Zhou, unpublished data) were mutated to phenylalanine by in vitro mutagenesis (Fig. 3-2 A, B). In addition, a lysine to arginine mutation at amino acid position 653 generated a mutant lacking any phosphorylation or kinase activity and therefore was included as a negative control (K653R). These EphA3 mutants and wild type EphA3 were each transiently expressed in HEK293A cells, and the cell lysates were incubated with sepharose beads conjugated with GST-Nck1SH2 protein. The kinase dead mutant EphA3-K653R failed to bind Nck1 SH2 domain (Fig. 3-3 B, lane 6), indicating that phosphorylation of the cytoplasmic tyrosine residues is necessary for binding (Fig. 3-3 A, lane 6). Among the tyrosine mutants, both Y596F and Y602F showed no binding to Nck1 SH2 domain similar to the K653R, while the others displayed normal binding (Figure 3-3 B, lane 1 to 5). Since Y596 and Y602 may regulate EphA3 kinase activity (Fig. 3-3 A, Lane 1-2), the loss of binding we observed could be due to either a lack of receptor phosphorylation, or the loss of the key tyrosine residues. To test these two possibilities, two additional mutants (Y596E and Y602E) were used, each with one tyrosine residue replaced by glutamic acid (Fig. 3-2 A, B). The glutamic acid replacement has been shown to mimic both the size and charge of a phosphorylated tyrosine and restore kinase activity on similar mutants [(Zisch et al. 2000), Fig. 3-3 A, lane 8-10]. Pulldown studies using these mutants showed that only Y602E failed to bind GST-Nck1SH2 protein although it exhibited normal levels of tyrosine phosphorylation (Fig. 3-3 B, lane 8-10). This 
observation indicates that Y602 is the key tyrosine residue that mediates direct Nck1 binding.

\section{EphA3 and Nck1 form a protein complex in ephrin-A5-stimulated cells.}

To examine whether Nck1 interacts with EphA3 in mammalian cells, EphA3 and HA tagged Nck1 were transiently expressed in HEK293A cells. The expression of both proteins was confirmed by Western blot analysis using anti-EphA3 antibody and anti-HA monoclonal antibody (Fig. 3-4). To conduct co-immunoprecipitation, the transfected cells were stimulated either with $2 \mu \mathrm{g} / \mathrm{ml}$ cross-linked ephrin-A5 or control IgG for 15 minutes, and lysed with lysis buffer. Nck1 protein was precipitated from the cell lysates with a mouse monoclonal anti-HA antibody. The immunoprecipitated proteins were analyzed by Western blot to determine if EphA3 was co-precipitated. The analysis revealed that from cell lysates with ephrin-A5 treatment, but not with control stimulation, a significant amount of EphA3 receptor co-precipitated with Nck1 (Fig. 3-4, lane 4 and 5). This observation indicates that EphA3 activation is required for EphA3-Nck1 binding in living cells. It is necessary to point out we did note a small amount of precipitated EphA3 from lysates of cells only transfected with EphA3 (Fig. 3-4, lane 3). This was probably due to the endogenous Nck1 expression in the HEK293A cell line (data not shown).

To further study interactions between Nck1 and EphA3, the sub-cellular localization of these two proteins was also examined. Cells transfected with EphA3 and HA-Nck1 were treated with cross-linked ephrin-A5 for 30 minutes and then fixed with $4 \%$ paraformaldehyde in PBS. Fixed cells were stained with a rabbit anti-EphA3 antibody 
followed by a FITC-conjugated anti-rabbit secondary antibody to label EphA3 receptor and a monoclonal anti-HA antibody followed by a Cy3-conjugated secondary antibody to mark Nck1 localization. It was found that both EphA3 and Nck1 were highly expressed in the transfected cells and there was considerable co-localization of two proteins in transfected 293 cells (Fig 3-5; yellow areas in A', and B'). Intriguingly, we also found there were some protein aggregates in the cytosol, which stained strongly of both EphA3 and Nck1 (Fig. 3-5, A-A'’). We speculate that these aggregates were endocytosed EphA3/Nck1 protein complex (Barbieri et al. 2004; Irie et al. 2005; Ceresa 2006). Indeed, previous studies have shown that at the end of Eph receptor signaling, the ligandreceptor dimers are cleaved and endocytosed for recycling (Mancia and Shapiro 2005).

\section{Nck1 mutants down-modulate ephrin-A5-mediated inhibition of cell migration.}

To examine whether Nck1 is important for EphA3 mediated functions, we tested the effects of EphA3 activation on cell migration and Nck1's role in this process. Two Nck1 dominant negative mutants and Nck1 wild type clone (generously provided by Dr. B. Mayer) were used in our study. The two Nck1 dominant negative mutants: Nck1R308K and Nck1W38/143/229K each contain mutations on key amino acid residues (Tanaka et al. 1995; Gupta and Mayer 1998). The R308K mutation disrupts the ability of the SH2 domain to bind to phosphotyrosine residues, while the W38/143/229K mutations abolish the ability of all three SH3 domains to interact with downstream targets containing proline-rich sequences. It was previously reported that both mutants inhibit functions of wild type Nck1 (Tanaka et al. 1995). 
HEK293A cells were transfected with EphA3WT alone or together with Nck1WT, Nck1R308K, or Nck1W38/143/229K. Twenty-four hours after transfection, the cells were plated on Transwell inserts $(8 \mu \mathrm{m}$ pore size) in serum free medium in the upper chamber. The lower compartment of the transwell apparatus was filled with complete culture medium with or without cross-linked ephrin-A5 added (Fig. 3-6 A). After overnight culture, quantification of the migration assay was performed with the aid of ImagePro program. Ten random pictures were taken of each insert on a microscope. The number of all cells that migrated through the membrane (T2) and the number of the transfected cells that migrated (T1) in each picture were both quantified. The efficiency of each individual transfection was determined separately: $\mathrm{R}=$ transfected cell/all cells. The migration ability $(\mathrm{M})$ of cells expressing different genes was then expressed using this formula: $\mathrm{M}=\mathrm{T} 1 /(\mathrm{T} 2 \times \mathrm{R})$. The $\mathrm{M}$ values of cells expressing different genes were normalized with EGFP control as 1 and plotted in Fig. 3-6 B.

Quantification showed that transient expression of EphA3WT caused a sharp decline in cell migration, compared to EGFP controls or EphA3KD. The decline of cell migration was found to be statistically very significant (student t-test, $\mathrm{p}<0.001$ ). Thus, EphA3 has a negative effect on cell migration and the kinase activity is indispensable to this regulation.

To examine whether Nck1 expression alone influences cell migration, we tested effects of expression of Nck1WT, Nck1R308K or Nck1W34/143/229K individually. The migration of cells expressing any Nck1 clone alone was similar to the EGFP control (Fig. 3-6 B), indicating that Nck1 expression alone did not affect cell migration. However, when Nck1R308K or Nck1W34/143/229K mutants were co-expressed with EphA3WT, 
they were able to partially block the inhibition of cell migration induced by ephrin-A5 treatment (Fig. 3-6 B). In contrast, Nck1WT had no such effect. The differences were found to be also very statistically significant (student t-test, $\mathrm{p}<0.001$ ). Collectively, these observations indicate that the interaction through $\mathrm{SH} 2$ and $\mathrm{SH} 3$ domains of $\mathrm{Nck} 1$ are both necessary for regulating cell migration of EphA3-expressing cells.

\section{Nck1 mutants inhibit ephrin-A5-induced cell process retraction.}

Ephrin-A5 stimulation in 293 cells has been shown to lead to the retraction of cellular processes when EphA3 receptor is expressed [(Lawrenson et al. 2002), Fig. 3-7, C-C']. This provides the basis of a convenient assay to examine whether Nck1 functions downstream of EphA3, and if dominant-negative Nck1 mutants affect EphA3 signaling. To conduct this assay, HEK293A cells were plated sparsely on $35 \mathrm{~mm}$ tissue culture dishes and transfected either with EphA3 alone, or EphA3 with wild type Nck1 (Nck1WT), Nck1R308K, or Nck1W38/143/229K. Forty-eight hours after transfection, cells were treated with $2 \mu \mathrm{g} / \mathrm{ml}$ cross-linked ephrin-A5 for one hour. Parallel cultures were treated with no ephrin-A5 as controls. In each culture dish, select cells with processes were photographed before and after treatment (Fig. 3-7 and Fig. 3-8). Using ImagePro program, we analyzed the images of the same groups of cells to determine how much process retraction occurred when cells were expressing different Nck1 genes. The total process length of a cell was measured before and after treatment: L1 and L2. Using the formula $\mathrm{E}=(\mathrm{L} 2-\mathrm{L} 1) / \mathrm{L} 1$, we calculated the process length change index $\mathrm{E}$ as a percentage of the initial total process length. The E values were then plotted in Fig. 3-9. 
In the graph, a positive number indicates process extension and a negative number indicates retraction.

Ephrin-A5 treatment of cells transfected with an empty EGFP vector did not cause any retraction of the cellular processes (Fig. 3-7, A and A'). On the contrary, on average, the branches extended about $4.7 \%(+/-3.9 \%)$. Ephrin treatment to cells transfected with the kinase-dead EphA3 also did not lead to any deleterious effects on the length of the processes (Fig. 3-7, B and B'). However, when the cells were transfected with EphA3WT, ephrin-A5 treatment induced significant process retractions (Fig. 3-7, C and C'). Quantification of the process length of treated cells showed that EphA3WT expressing cells retracted their processes by about $24.3 \%(+/-5.0 \%)$. In comparison, cells expressing EphA3KD extended about 0.4\% (+/-3.3\%) during the same period of time. These statistically significant differences (student t-test, $\mathrm{p}<0.05$ ) suggest kinase activity of EphA3 is necessary to the ephrin-induced inhibition of process extension.

Co-transfection of Nck1WT with EphA3WT did not inhibit process retraction of EphA3WT-expressing cells in response to ephrin-A5 challenge (Fig. 3-8, A and A'). The process length was shortened by about $22 \%(+/-3.0 \%)$ similar to cells expressing only EphA3WT. However, when either Nck1R308K, or Nck1W38/143/229K, was coexpressed with EphA3WT, the process retraction induced by ephrin-A5 was significantly less (Fig. 3-8, B-C'). In the presence of Nck1R308K, or Nck1W38/143/229K, the decrease of process length of cells expressing EphA3WT was only 9.2\% (+/- 3.9\%) and $6.5 \%(+/-2.1 \%)$, respectively. These results suggest that Nck1 protein is important in EphA3 signal transduction and the interaction mediated by both $\mathrm{SH} 2$ and $\mathrm{SH} 3$ domains are critical. 


\section{Discussion}

The present study confirms the hypothesis that Nck1 is a strong binding partner of the EphA3 receptor, and demonstrates that the binding is mediated by a specific interaction between the Nck1 SH2 domain and the phosphotyrosine at 602 on EphA3. Inhibition of Nck1 function using dominant-negative mutants tempers inhibitory effects of EphA3 on cell migration and process extension, suggesting that Nck1 is a key downstream mediator of EphA3 functions.

\section{Nck1 interacts with activated EphA3.}

As described earlier, Nck1 has been found to interact with both EphA3 and EphA5 receptors. An early study by Stein et al. has showed that Nck1 binds to the intracellular domain of EphB1 (Stein et al. 1998). It was also reported that EphB2 bound to p62 dok after activation and formed a protein complex that included both RasGAP and Nck1 (Holland et al. 1997). These data together lend support to the notion that Nck1 function downstream of both EphA and EphB receptors and mediate key functions.

Consistent with the observation in the yeast two-hybrid experiments, we found that Nck1 protein was co-precipitated with EphA3 from transfected cells, and determined the interaction was mediated by Nck1 SH2 domain. These findings indicate that the interaction of Nck1 with EphA3 is similar to its interaction with EGF receptor and Crkassociated substrate (p130 $0^{\text {Cas}}$ ) in PDGF-stimulated cells (Roche et al. 1996; Tang et al. 1997; Rivera et al. 2006), but different from its binding to DCC and Robo, which occurs through the SH3 domains ( $\mathrm{Li}$ et al. 2002). Furthermore, mutagenesis coupled with in 
vitro pulldown experiments conducted in this study allowed us to identify key interaction sites on EphA3 for Nck1. It was found that neither EphA3-Y602F nor EphA3-Y602E bound Nck1, although both mutants exhibited normal kinase activity as measured by autophosphorylation and phosphorylation of exogenous substrate (Fig. 3-3 A and G. Shi and R. Zhou, unpublished observations). Thus, tyrosine residue 602 of EphA3 is indispensable for Nck1 binding and likely to be responsible for most, if not all, of the interaction. In addition, Nck1 SH2 domain has been shown to bind strongly to the sequence of "pTyr-hydrophilic-hydrophilic-Pro/Ile" through an unbiased screen in a degenerate phosphopeptide library (Songyang et al. 1993). The EphA3 amino acid sequence behind tyrosine 602 (pYEDP) fits this description. Interestingly, earlier studies showed that Nck1 interacts with EphB1 through the SH2 domain and Y594 (the equivalent of Y596 of EphA3) (Stein et al. 1998), indicating that there are variations in interaction mechanisms among different Eph receptors.

We also showed that activation of EphA3 by ephrin-A5 treatment induced the EphA3-Nck1 interaction. In the absence of ephrin-A5 stimulation, no EphA3 was coimmunoprecipitated by anti-Nck1 antibody from transfected 293A cells. Protein subcellular co-localization studies were consistent with the results from the coimmunoprecipitation experiments. Considerable amount of Nck1, although not all, was co-localized with EphA3 receptors in transfected cells, especially on the plasma membrane.

Similar to EphA3, EphA5 was found to bind Nck1 through the yeast two-hybrid screen. Since EphA3 and EphA5 share high homology with each other (Zhou 1998), we speculate that the binding between Nck1 and EphA5 is also through the SH2 domain and 
tyrosine 656 (the equivalent of 602 of EphA3). It is likely that other EphA receptors also bind to Nck1 through this conserved tyrosine residue in the juxtamembrane domain. However, more research has to be conducted to confirm these speculations.

\section{Nck1 mediates regulation of cell migration and process retraction by EphA3 activation.}

In both process retraction and migration assays, we have shown that the overexpression of either dominant negative Nck1 mutant is able to inhibit effects of EphA3 activation. In contrast, Nck1 wild type has no such effects. Since the dominant negative clones are deficient in either SH2-mediated binding or SH3-mediated binding (Tanaka et al. 1995; Gupta and Mayer 1998), our observations suggest that both the recruitment of Nck1 to activated EphA3 through the SH2 domain and the interaction with downstream effectors through the SH3 domains are important steps in the signal transduction of EphA3 (Fig. 3-10). A potential concern in using dominant negative mutants is that the effects observed from these studies were due to non-specific inhibition of binding of other SH2 or SH3 domain-containing proteins by the mutants. However, if the effects of the mutants were non-specific, and Nck1 had no role in EphA3 signaling, it is expected that the expression of wild type Nck1 would also block EphA3 function. Our observation that wild type Nck1 did not block EphA3 function indicates that the mutants specifically inhibit Nck1 function and Nck1 is an important signaling intermediary downstream of the Eph receptor signaling.

Up to this date, Nck1 has been shown to interact with more than 30 proteins through the SH3 domains, most of which have been implicated in actin cytoskeleton re- 
organization (Li et al. 2001). These interactions strongly support for a critical role of Nck1 in regulating Eph regulated cell migration and process outgrowth. Indeed, clustering of Nck1 SH3 domain alone was found to be sufficient to induce localized actin polymerization (Rivera et al. 2006), suggesting that the SH3 domains mediate actin cytoskeleton regulation through effectors.

Nck1 interacts with the p21-activated kinase (PAK1) via its second SH3 domain (Galisteo et al. 1996; Lu et al. 1997; Zhao et al. 2000) and translocate PAK1 from the cytosol to plasma membrane. As a result, PAK1 is brought in close proximity of Rac1 and cdc42 and activated (Bokoch 2003). Active PAK1 phosphorylates substrates such as LIM (Lin-11, Isl-1 and Mec-3 domain) kinase, which in turn inactivates actin depolymerizing factor (ADF) family proteins. Members of ADF family such as cofilin and myosin light chain kinase (MLCK) are important regulators of actin/myosin organization (Edwards et al. 1999; Sanders et al. 1999). As a result, inactivation of PAK1 leads to cofilin dephosphorylation and actin depolymerization. Indeed, PAK1 activity is reduced in ephrin-A1 treated cortical neurons (Shamah et al. 2001), although it is not known if ADF/cofilin activity is increased. The existence of a Nck1-PAK1 pathway is further supported by genetic data from Drosophila: the guidance and targeting of photoreceptor growth cones requires activity of both Nck1 and PAK homologs (Garrity et al. 1996; Hing et al. 1999). It would be interesting to examine whether ephrin-induced inhibition of cell migration and process outgrowth is mediated through the Nck1-PAK1 pathway.

Another important group of Nck1 binding proteins are the WASP family proteins such as WASP and N-WASP (Rohatgi et al. 2001; Rivera et al. 2004; Samarin 2005; 
Takenawa and Suetsugu 2007). WASP mutations were first discovered in studies on an X chromosome linked immuno-deficient disease (Derry et al. 1994), which was characterized by cell abnormalities and actin cytoskeleton defects. Highly enriched in the brain, WASP proteins activate actin filament restructuring (Zalevsky et al. 2001)and regulate neurite growth (Takenawa and Suetsugu 2007). The WASP family proteins possess a C-terminal motif that binds to Arp2/3, a protein that mediates actin polymerization (Machesky et al. 1999; Rohatgi et al. 2001; Ibarra et al. 2005) . When Nck1 SH3 domain was over-expressed in cells with N-WASP and Arp2/3, an unusually high rate of actin nucleation was observed (Rohatgi et al. 2001). In addition, WIP, a WASP interacting protein, was also found to bind to Nck1 through a site different from WASP (Anton et al. 1998). WIP has been shown to associate with profilin, an actin cytoskeleton regulatory protein. Thus, Nck1 may function through the WASP-WIP pathway to regulate cell morphology and migration following EphA receptor activation.

A recent study reported that EphB stimulation led to the formation of a protein complex that included Nck1, NIK, dok1, and RasGAP. Consequently, active NIK turned on the c-Jun amino-terminal kinase (JNK) pathway (Becker et al. 2000). Interestingly, when the Drosophila NIK homolog, Misshapen was mutated, the photoreceptor axon targeting was disrupted ( $\mathrm{Su}$ et al. 1998), in a manner similar to the phenotype of Dock (Nck1 homolog in Drosophila) knockout animals (Ruan et al. 1999). It was therefore proposed that NIK was directly involved in Nck1 mediated axon guidance downstream of Eph receptors.

However, in the present study, neither Nck1 mutant was able to completely inhibit EphA3 effects in the assays we used. It is possible that complete inhibition requires high 
levels of expression of the mutant proteins, which were not achieved in our studies. Alternately, EphA3 might exert its effects in part through Nck1-independent pathways, likely through other SH2/SH3 domain proteins like Grb2, RasGAP, and Crk (Stein et al. 1996; Holland et al. 1997; Lawrenson et al. 2002). Another possibility is that Nck2, the other member in the Nck family plays a redundant role in EphA3 signaling since it shares high homology with Nck1 (Chen et al. 2000; Buday et al. 2002; Bladt et al. 2003). It has been reported that Nck2 binds to Abi-1, Axin, and CAP, all of which are involved in cytoskeleton regulation. In addition, Nck2 also regulates ILK (integrin-linked kinase) (Tu et al. 1998; Buday et al. 2002; Xu et al. 2005), providing a link from EphA receptors to integrin signaling. 
A.

Nck1

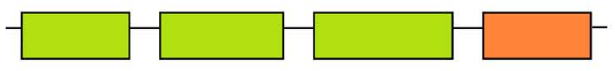

GST only $\square$

GST-Nck1 SH2 $\square$

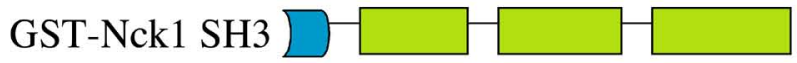

GST-Nck1

full length

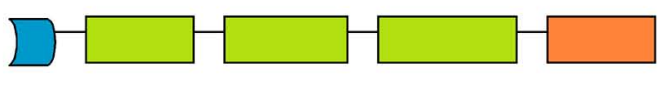

B.

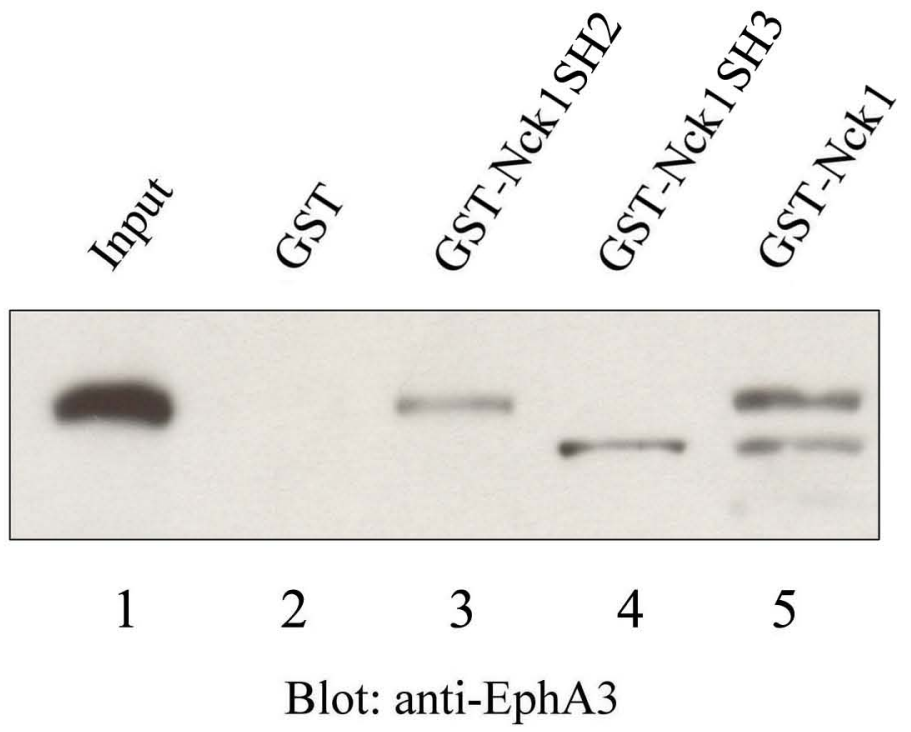


Figure 3-1. Interaction of Nck1 with EphA3 through SH2 domain.

(A) Domain structure of wild type Nck1 protein and Nck1 fusion proteins used in GST pulldown assay. (B) Identification of the EphA3 binding domain of Nck1. The GST fusion proteins were incubated with the lysates of HEK293A cells expressing EphA3 protein. The precipitated protein complex was analyzed by Western blot with anti-EphA3 antibody. "Input" shows the total EphA3 level in the whole cell lysate of transfected HEK 293A cells. 
A.

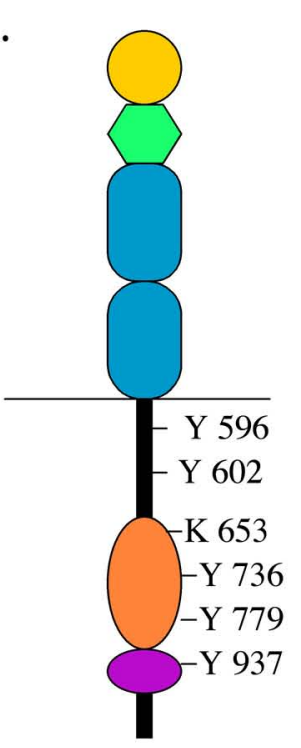

B.

\begin{tabular}{|c|c|c|c|c|}
\hline Location & Domain & Tyr $->$ Phe & Tyr->Glu & Lys->Arg \\
\hline Tyr 596 & Juxtamembrane domain & Y596F & Y596E & \\
\hline Tyr 602 & Juxtamembrane domain & Y602F & Y602E & \\
\hline Tyr 736 & Kinase domain & Y736F & & \\
\hline Tyr 779 & Kinase domain & Y779F & & \\
\hline Tyr 937 & SAM domain & Y937F & & \\
\hline Lys 653 & Kinase domain & & & K653R \\
\hline
\end{tabular}


Figure 3-2. A series of EphA3 mutants containing single tyrosine mutations.

(A) Map of the mutated residues on the EphA3 receptor. (B) Table summarizing all of the mutants used in this study. 

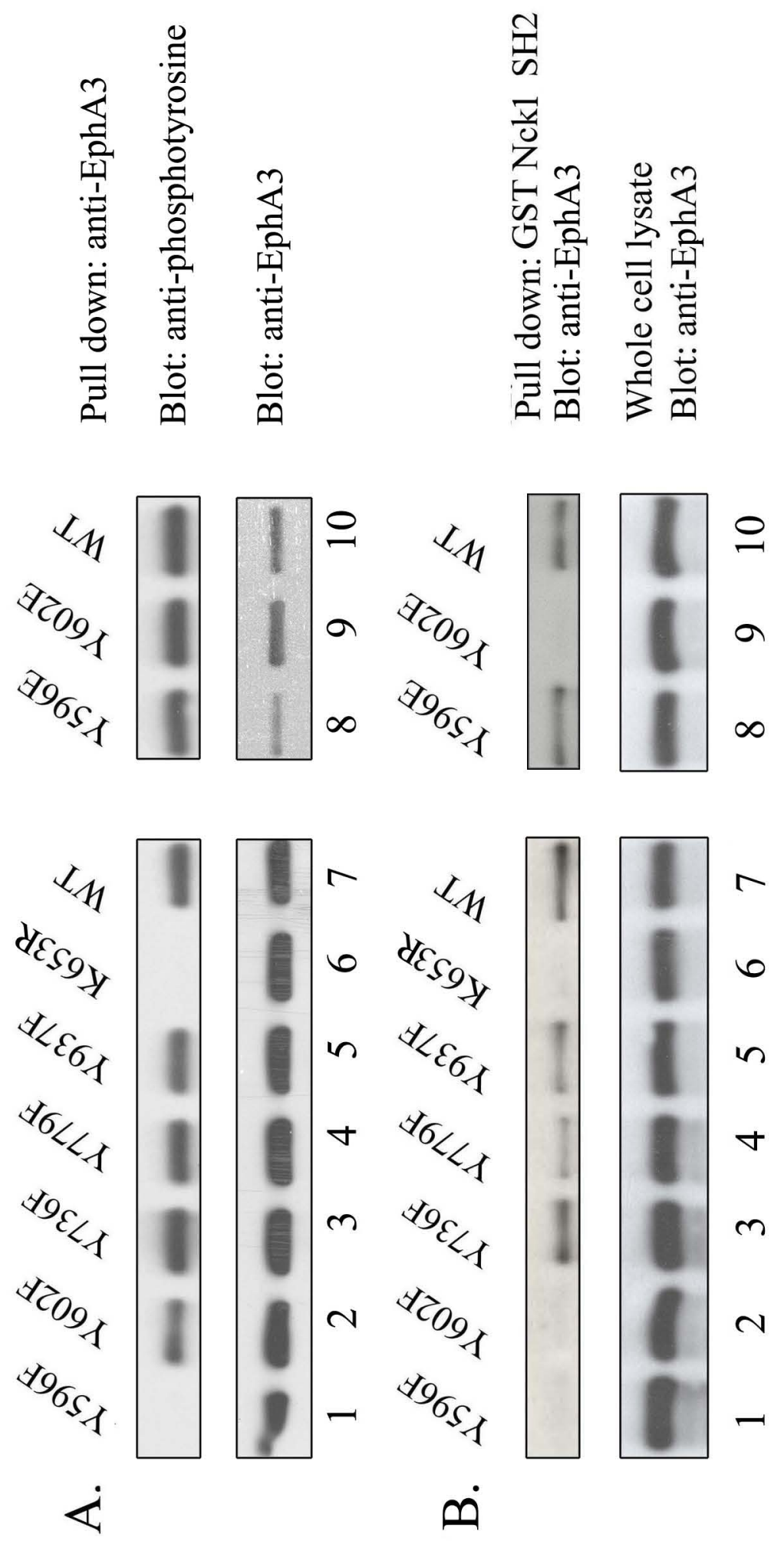
Figure 3-3. Identification of Tyr602 as the Nck1 binding tyrosine residue on EphA3. (A) Auto-phosphorylation of EphA3 mutants. Five tyrosine-to-phenylalanine mutants (Y596F, Y602F, Y736F, Y779F, Y937F), two tyrosine-to-glutamic acid mutants (Y596E, Y602E), a kinase inactive mutant (K653R) and wild type EphA3 (WT) were expressed individually in 293A cells. EphA3 proteins were immunoprecipitated and analyzed by Western blot with anti-phosphotyrosine antibody and anti-EphA3 antibody. (B) Y602 mutation inactivates Nck1 binding as shown by the GST pulldown assays using GST$\mathrm{NckSH} 2$ fusion protein. 


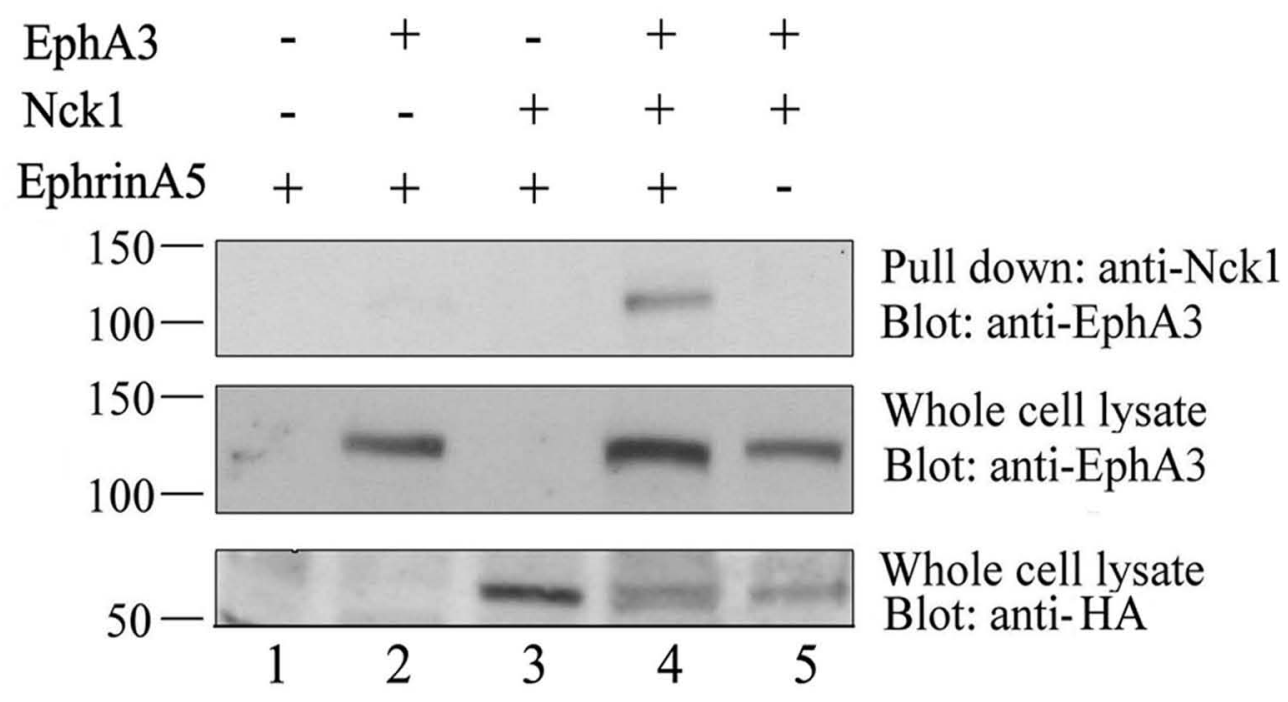


Figure 3-4. Precipitation of EphA3 by Nck1 antibody in 293A cells.

EphA3 and Nck1 were co-transfected into 293A cells and Nck1 protein complex was precipitated with a monoclonal anti-Nck1 antibody. The immunoprecipitates were analyzed by Western blot. EphA3 was precipitated by Nck1 antibody only when the cotransfected cells were stimulated with ephrin-A5. 

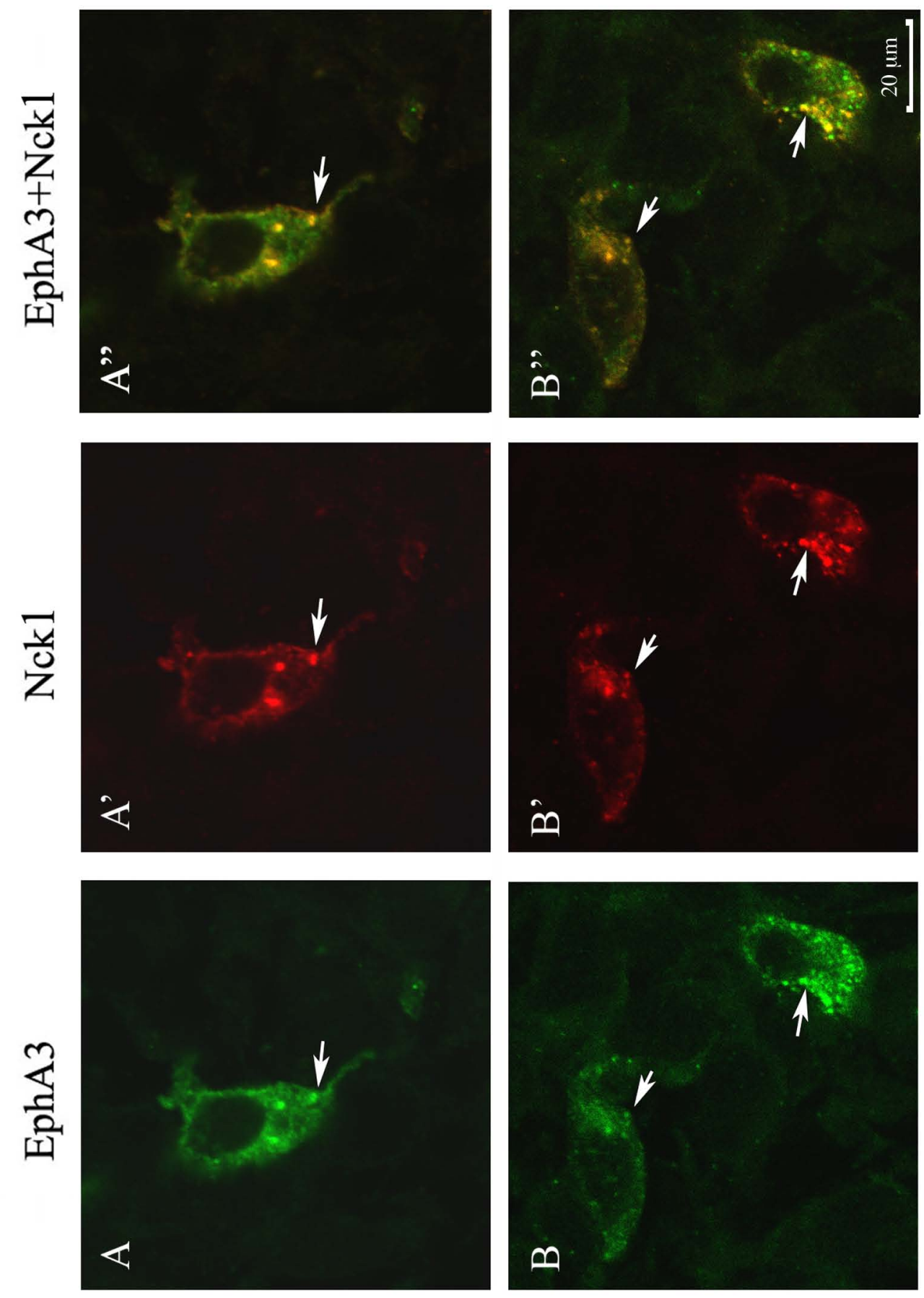
Figure 3-5. Co-localization of Nck1 and EphA3 in transfected HEK 293A cells.

Two groups of cells, shown in panel A-A', and B-B', were stained with FITC-antiEphA3 (Panels A and B) and Cy3-anti-Nck1 (Panels A' and B'). Superimposition of the two single channel pictures (Panels A'" and B') shows the co-localization of EphA3 and Nck1 protein, especially on the plasma membrane, as indicated by white arrows. 
A.

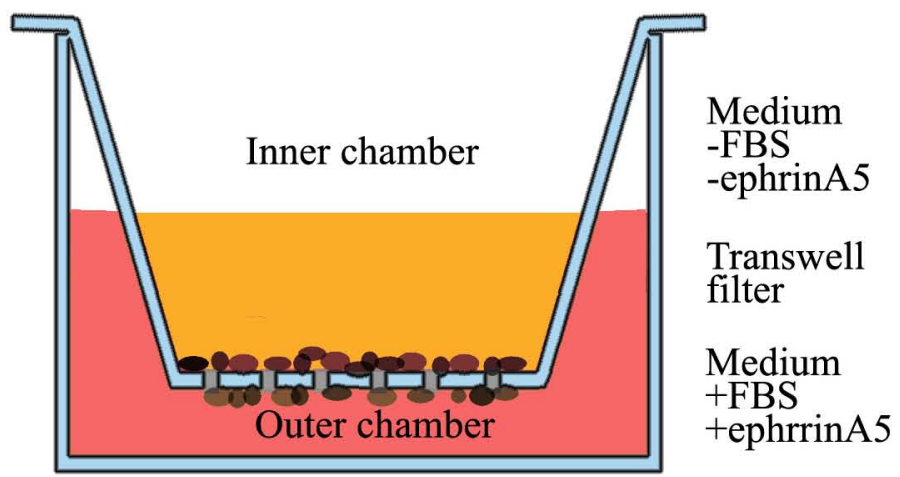

B.

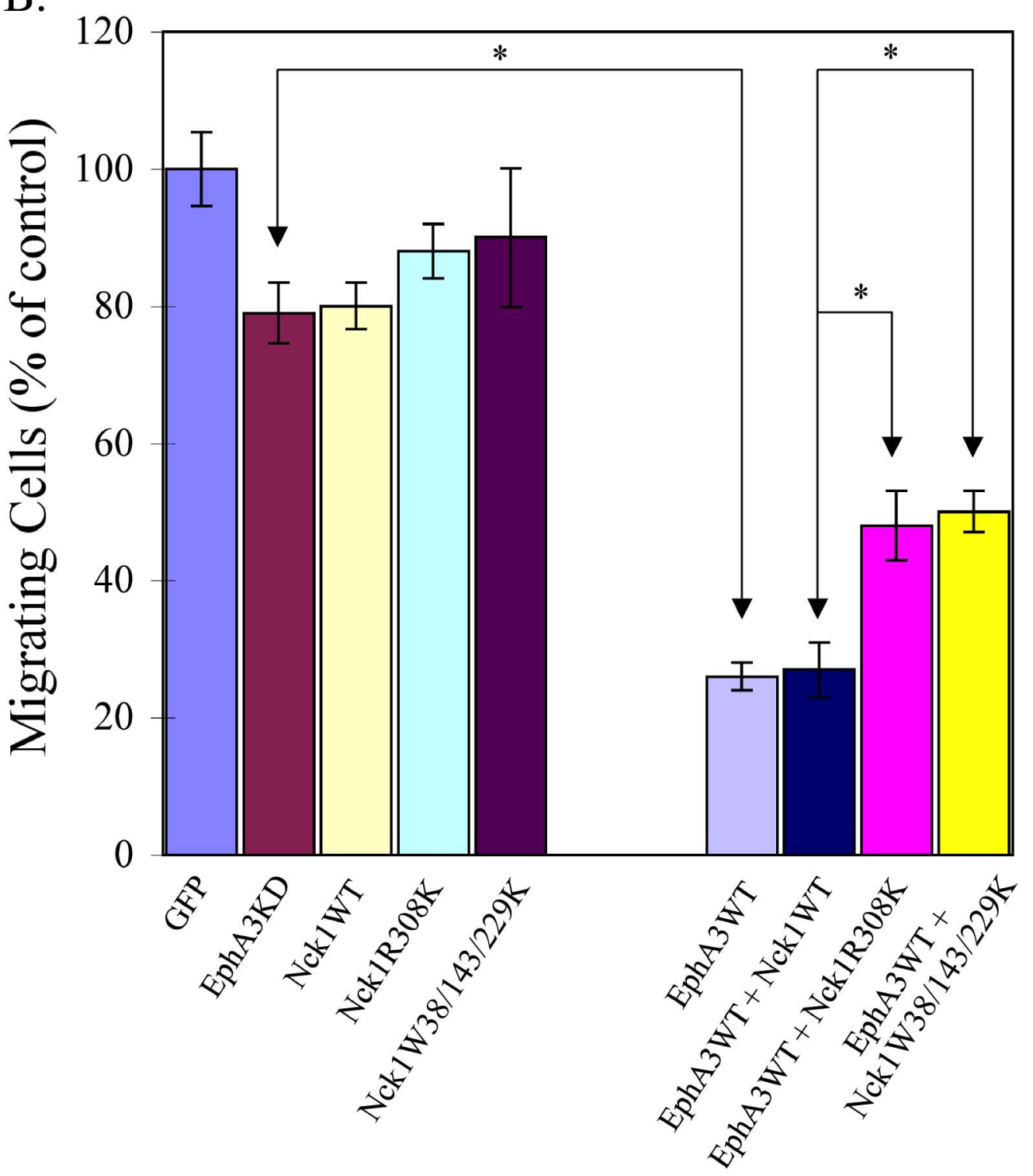


Figure 3-6. EphA3-induced inhibition of cell migration mediated by Nck1.

(A) The transwell apparatus used in migration assay. HEK293A cells were transiently transfected with EGFP, EphA3KD, Nck1WT, Nck1R308K, Nck1W38/143/229K, EphA3WT, EphA3WT+Nck1WT, EphA3WT+Nck1R308K and EphA3WT+Nck1W38/143/229K. Twenty-four hours later, cells were plated in transwells and a migration assay was performed as shown in the graph. (B) Quantification data is summarized in the graph. Each bar in the graph represents the migratory ability (M) of transfected cells, normalized to EGFP expressing cells. Asterisks indicate a statistically significant difference (student t-test, $\mathrm{p}<0.001$ ), when compared to proper controls (EphA3KD for EphA3WT, EphA3WT+Nck1 for EphA3WT+Nck1R308K and EphA3WT+Nck1W38/143/229K). 


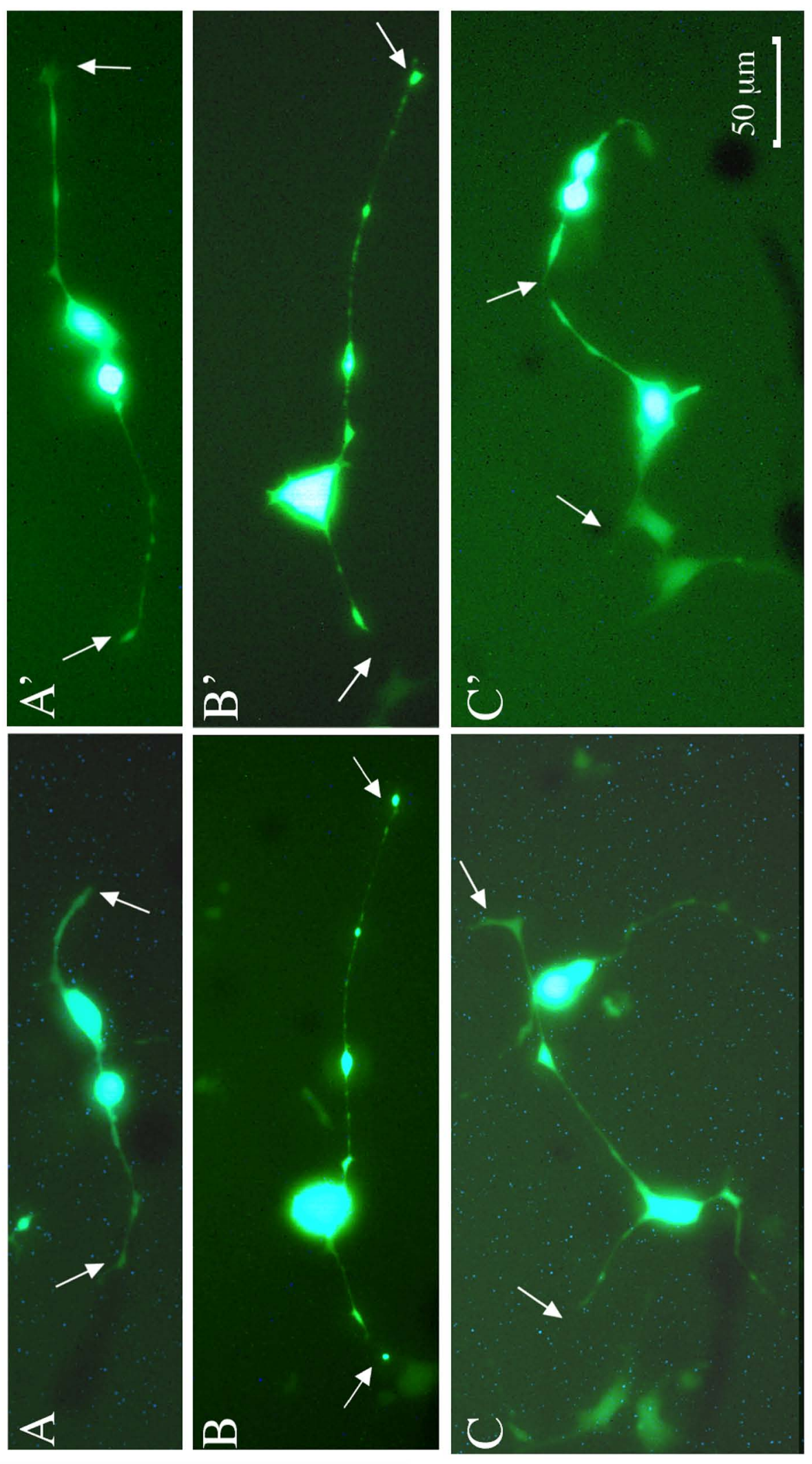


Figure 3-7. Cell process retraction induced by activation of EphA3.

HEK293A cells were transfected with EGFP (A-A'), or EphA3KD (B-B') or EphA3WT (C-C'). Forty-eight hours after transfection, the cells were treated with cross-linked ephrin-A5 for 60 minutes. Select cells were photographed before and after the treatment. The length of all identifiable processes on each cell was measured. Arrows indicate the end positions of the processes in each picture. 


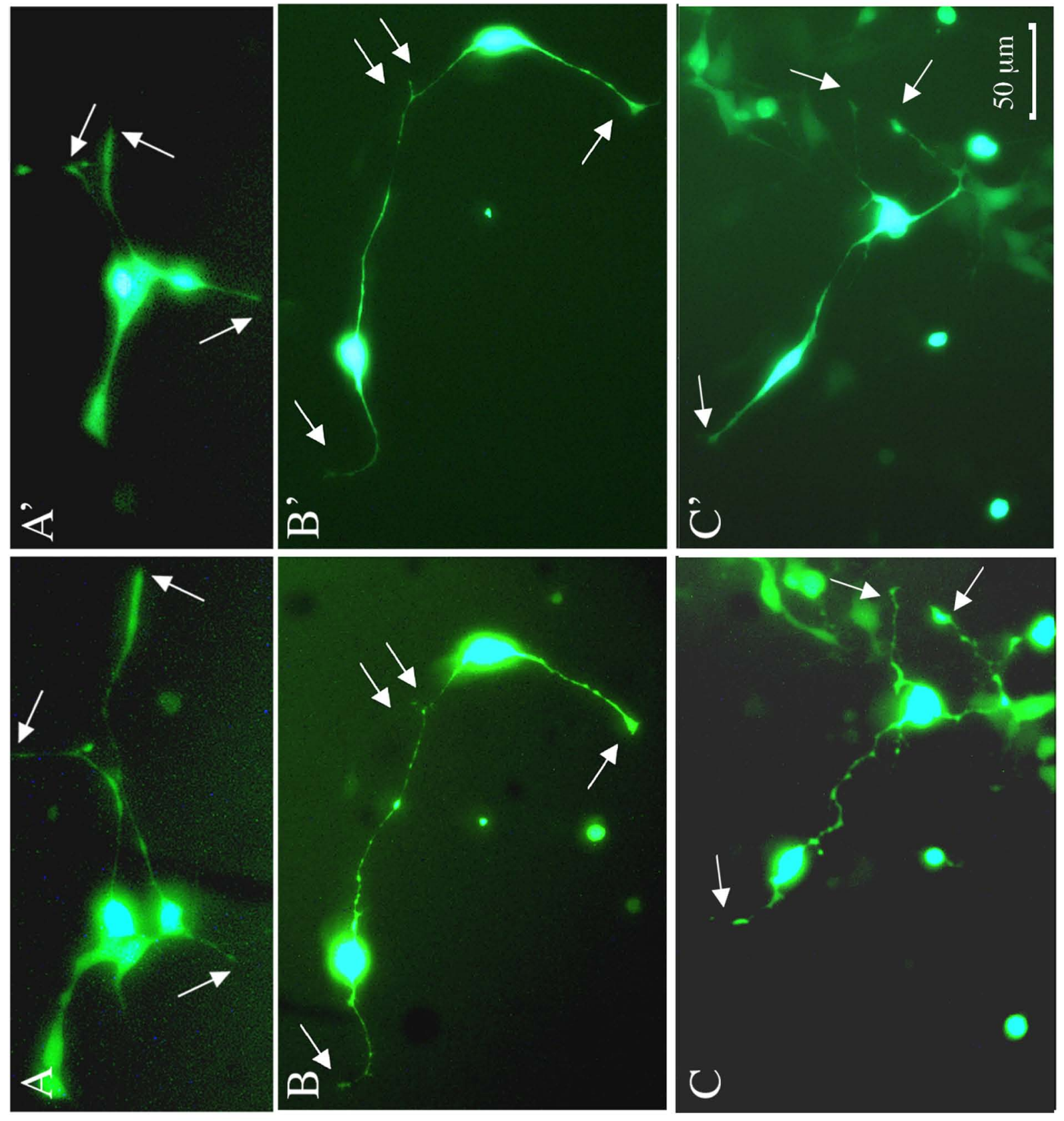


Figure 3-8. Disruption of EphA3-induced process retraction by Nck1 DN genes. Nck1WT (A-A'), Nck1R308K (B-B'), or Nck1W38/143/229K (C-C') were cotransfected with EphA3WT in order to examine the effects of Nck1 clones in process retraction induced by ephrin-A5 stimulation. Arrows show the end positions of cellular processes in each picture. 


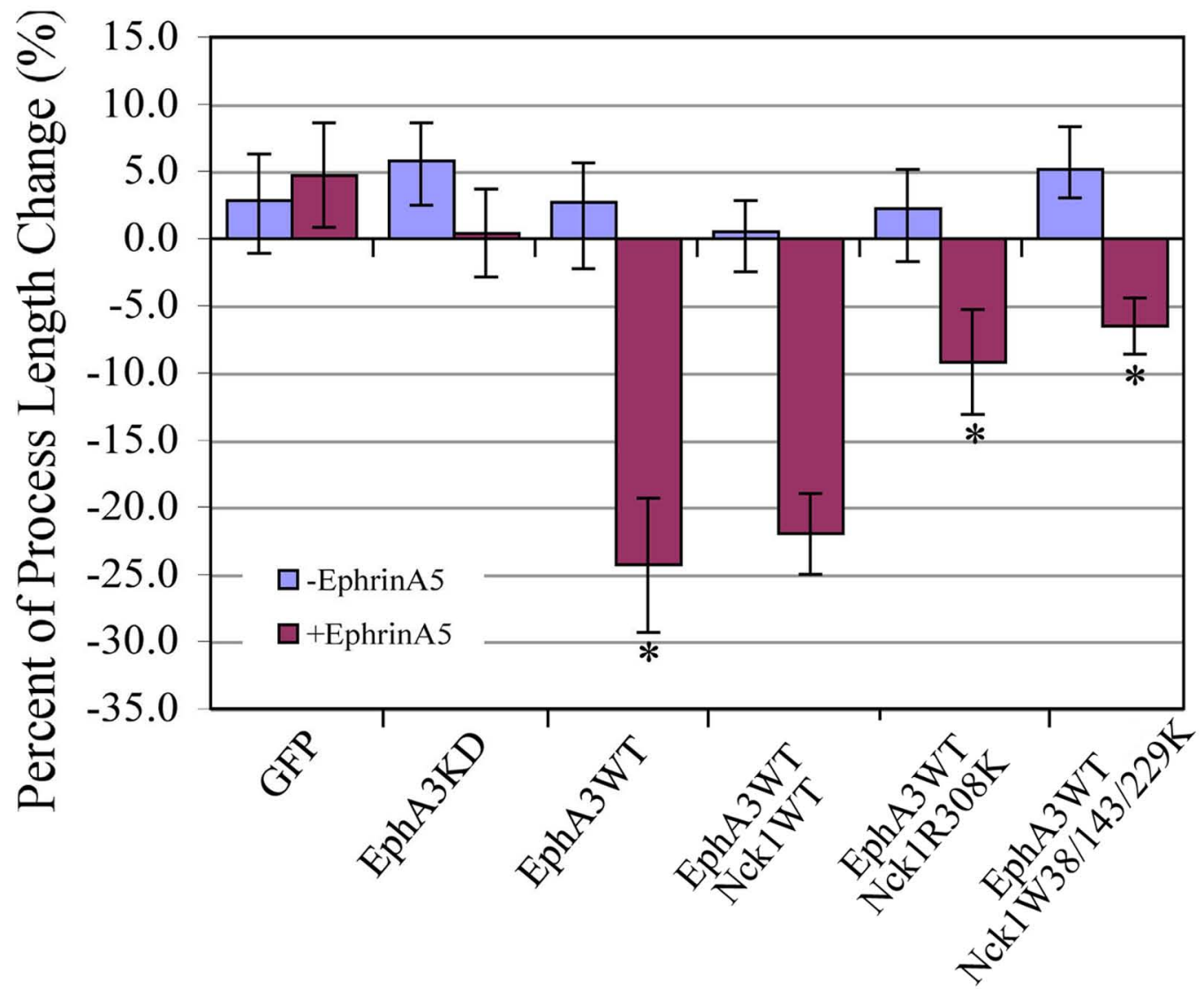


Figure 3-9. Quantification of Nck1 mutant effects on EphA3-induced process retraction.

Plotted are changes of process length after ephrin-A5 treatment, normalized to EGFP control cells. A positive number indicates extension during treatment, while a negative value shows retraction of the processes. Asterisks indicate statistical significant differences when compared to proper controls (EphA3KD for EphA3WT, EphA3WT+Nck1WT for EphA3WT+Nck1R308K and EphA3WT+Nck1W38/143/229K; student t-test, $\mathrm{p}<0.05)$. 


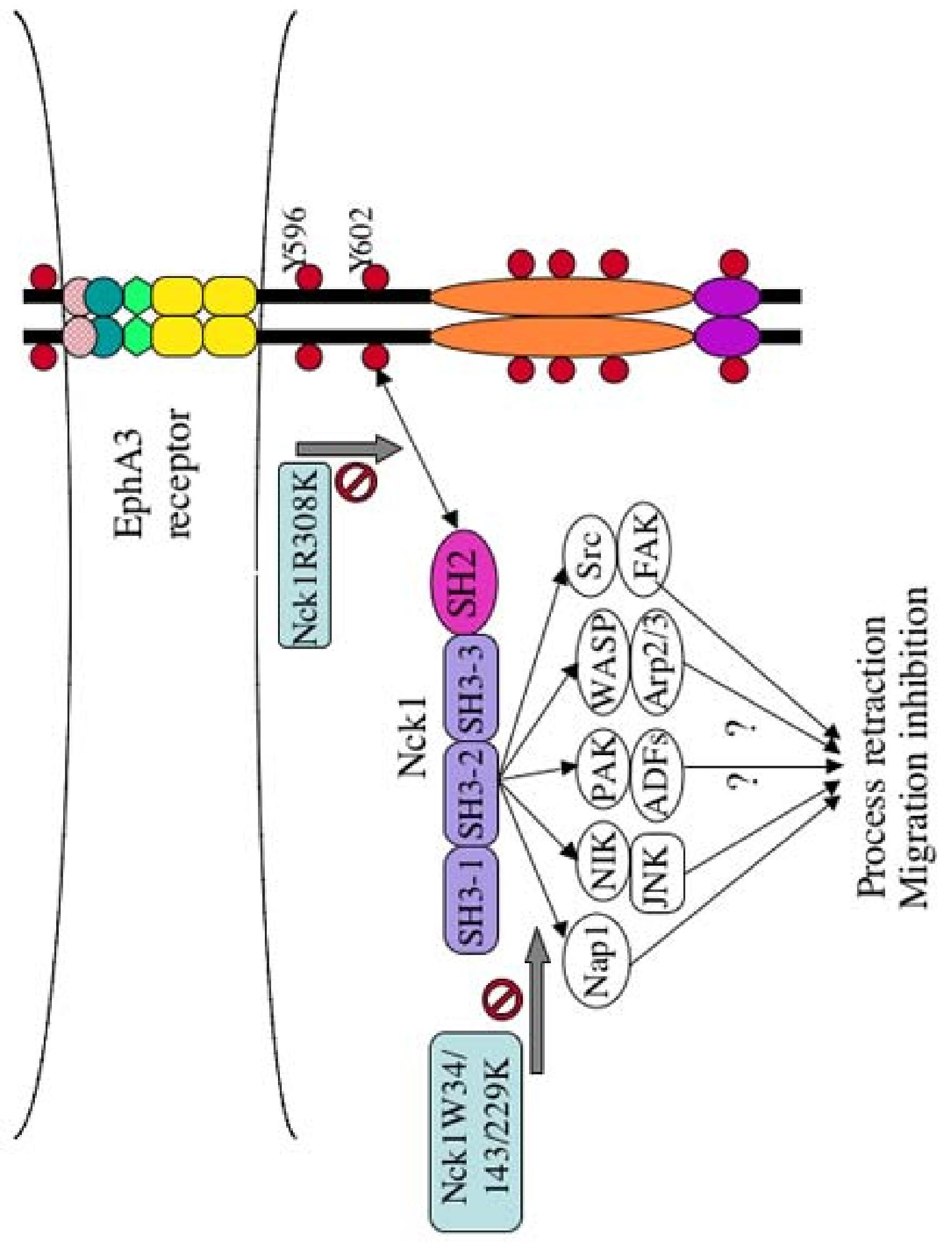


Figure 3-10. A model of the effects of Nck1 DN genes in EphA3 signaling. Nck1R308K and Nck1W34/143/229K are Nck1 clones that show disrupted binding through the SH2 domain or all three SH3 domains, respectively. The overexpression of either protein overrides the normal function of endogenous Nck1 and leads to the disruption of EphA3 mediated functions such as process retraction and inhibition of cell migration. 


\section{Chapter IV. Analysis of EphA3-Nck1 binding in neurons and}

\section{assessment of Nck1 in hippocampal neuron growth cone}

\section{collapse}

\section{Introduction}

Thus far, all the research we have conducted on Nck1 provides convincing evidence on the significant role Nck1 plays in EphA mediated signaling. In fibroblast cells expressing both proteins, Nck1 molecules in the cytosol respond to EphA3 stimulation by binding to phosphorylated tyrosine 602 in the juxtamembrane domain and modulating downstream pathways. Dominant negative forms of Nck1, when overexpressed, disrupt this process and consequently disrupt EphA3 regulated cell responses such as process retraction and cell migration inhibition.

This study, however, has only tested systems in which EphA3 and Nck1 were overexpressed artificially. In order to show the functional significance of binding between endogenous EphA3 and Nck1, we set out to investigate the possible role of Nck1 in EphA3 mediated signaling in the developing nervous system. EphA3 is known to be highly expressed in late embryonic hippocampus and cortex (Yue et al. 2002), a time when the formation of critical neural circuits occurs. EphA3 regulates various important neural events such as axon guidance and axon fasciculation (Winslow et al. 1995;

Friedman and O'Leary 1996). It is likely that Nck1 is involved in the regulation of these functions. Indeed, the initial yeast two-hybrid screen was performed in an embryonic mouse brain cDNA library. Taken together, these data prompted us to hypothesize that 
Nck1 adaptor protein plays a critical role in neurons, linking extracellular signal through Eph receptors to downstream functional pathways such as the PAK pathway, the Sos/Ras pathway and effectors such as WASP and NIK. More specifically, we speculate that the disruption of Nck1 association with either activated EphA receptor or downstream effectors would render the signaling cascade dysfunctional and cause major defects in EphA3 signaling in the developing nervous system.

E18 rat embryonic brains were dissected and cortical and hippocampal neurons were isolated for this study. The endogenous expression of both EphA3 and Nck1 was evaluated by Western blot. Immunoprecipitation and immunostaining methods were applied to assess whether Nck1 directly associates with activated EphA3 receptor.

After studying EphA3-Nck1 binding in the neuron cultures, a growth cone collapse assay was employed to test in neurons if dominant negative Nck1 mutants were able to disrupt cellular events that are regulated by EphA3 receptor, similar to what occurs in transfected HEK293A cells.

\section{Results}

\section{The expression of Nck1 in primary neuron culture}

In the embryonic rat, EphA3 expression emerges at E12 at high levels in the subventricular zone and persists through adulthood (Kilpatrick et al. 1996; Ernst et al. 1998). In addition, motor neurons and retinal neurons also express EphA3 (Sajjadi and Pasquale 1993; Marin et al. 2001; Otal et al. 2006). Nck1 is expressed in a large variety of tissues including heart, brain, testis, liver. Most important to this study, EphA3 is expressed at a stable level in the developing rat brain (Li et al. 1992; Park and Rhee 1992). The question 
remains, however, if Nck1 is co-expressed with EphA3 in the same group of developing neuronal cells.

To answer this question, the cerebral cortices and the hippocampi were dissected from E18 rat embryonic brain. The dissociated cortical and hippocampal neurons were cultured on Poly-D-lysine coated dishes (Fig. 4-1 A). Two days after initial plating, the neuron cultures were lysed and the total cell lysates were separated on SDS-PAGE gel and analyzed by Western blot. Both anti-EphA3 antibody and anti-Nck1 antibody were used to assess the expression level of the two proteins at E18 (Fig. 4-1 B).

The Western blot data revealed that EphA3 receptor was expressed in both cortical neurons and hippocampal neurons of rats at E18, consistent with previous reports (Martinez and Soriano 2005; Otal et al. 2006). In addition, we observed that there was substantial expression of Nck1 in both cortical neurons and hippocampal neurons as well. These findings confirm that Nck1 and EphA3 are co-expressed in developing cortical and hippocampal neurons.

\section{EphA3 and Nck1 interact in hippocampal neurons and cortical neurons}

In Chapter III, we showed by immunostaining and immunoprecipitation methods, that ephrin-A5 stimulation promotes the binding of Nck1 with activated EphA3 receptor. Similarly, we applied these two techniques to analyze Nck1-EphA3 binding in primary neuron cultures.

E18 embryonic rat hippocampal neurons were cultured for two days before they were treated with cross-linked ephrin-A5 and fixed. Anti-EphA3 antibody, followed by FITC conjugated secondary antibody, was first used to label EphA3 localization. Anti-Nck1 
antibody and $\mathrm{Cy} 3$ conjugated secondary antibody were then used to mark Nck1 protein. The stained neurons were photographed under a fluorescent microscope. The staining revealed that both proteins were expressed highly and they displayed a high degree of colocalization on the plasma membrane, especially on the growth cones as marked by the yellow areas (Fig. 4-2).

Immunoprecipitation was also used to ascertain the putative association of endogenous EphA3 and Nck1 in E18 neurons. One limitation of this method is a large quantity of total cell lysates is usually required to perform this assay. To circumvent this limitation, embryonic cortical neurons instead of hippocampal neurons were used, since the cortical neurons exist in greater abundance in rats. The cortical neurons were cultured for two days on Poly-D-lysine coated dishes and then treated with or without $1 \mu \mathrm{g} / \mathrm{ml}$ dimerized ephrin-A5 for 30 minutes before being lysed. Cell lysates were then precipitated with a mouse anti-Nck1 antibody. As a negative control, ephrin-A5 stimulated cortical neuron lysates were incubated with control mouse IgG instead of the Nck1 specific antibody. The precipitated proteins were separated by SDS-PAGE and analyzed by Western blot (Fig. 4-3). Total neuron lysates were included to show expression of both EphA3 and Nck1.

In this experiment, Nck1 antibody precipitated high level of EphA3 from stimulated cell lysates (Fig. 4-3, lane 2). By contrast, neither precipitating from untreated cell lysates with Nck1 antibody nor precipitating from stimulated cell lysates with control IgG brought down EphA3 (Fig. 4-3, lane 1 and 3). These observations collectively show that Nck1 binds to activated EphA3 and ephrin-A5 stimulation is a prerequisite to this 
binding. Furthermore, the confirmation of EphA3-Nck1 in neuron lysates suggests that Nck1 is an important factor in EphA3 regulated signaling cascades in neurons.

\section{Ephrin-A5 induces growth cone collapse in a hippocampal neuron culture}

Dissociated hippocampal neurons in culture adopt the characteristic neuronal morphology very quickly upon plating. This is characterized by the extension of long axons on one side of the cell body and the growth of multiple short dendrites on the other side as visualized by staining the cells with Texas-red Phallodin (Fig. 4-4 A). The overall morphology of the neurons is best visualized by staining with Texas-red Phallodin, a dye specific for actin filaments. Growth cones, the hand-like structures located at the end of neurites, stain heavily with Texas-red because of the high actin content. (Fig. 4-4 B). Because growth cones are responsible for guiding the axons through the environment in response to guidance cues, they are pivotal to axon guidance. A typical growth cone consists of two parts: the central domain and the peripheral domain (Fig. 4-4 C). The central domain, which connects the neurite and the growth cone, is enriched with microtubules and organelles. The peripheral domain, with its high actin cytoskeleton content, is the functional part of the growth cone that detects guidance cues and undergoes rapid changes. Upon close inspection, the peripheral domain is composed of relatively stable sheet-like expansions called "lamellapodia" and fine processes called "filopodia" that extend and retract constantly (Goldberg and Burmeister 1986; Forscher et al. 1987; Orioli and Klein 1997; Wilkinson 2000). Growth cones are known to contain an abundance of receptors and signaling molecules. Working together closely, these proteins orchestrate rapid responses of to extracellular stimuli. 
Expression of EphA3 receptor in both hippocampal neurons and cortical neurons enables these neurons to respond to ephrin-A ligand stimulation efficiently. The receptor, as shown earlier, is highly localized to the growth cone and along the neurites. Thus, ephrin-A treatment triggers an immediate response on the growth cone and continues on the axon and soma (Meima et al. 1997; Meima et al. 1997). This immediate response is best exemplified by a growth cone collapse assay.

E18 hippocampal neuron explants were cultured in serum-free neurobasal medium for two days. Prior to ephrin-A5 treatment, cultured hippocampal neurons usually possessed characteristic growth cones, exhibiting an active exploration of the surroundings (Fig. 4-4 E). However, within as little as 15 minutes of ephrin stimulation, dramatic morphological changes were observed on a majority of the growth cones (Fig. 4-4 F). Instead of maintaining the hand-like morphology, the growth cones collapsed into a lean tapered shape (Fig. 4-4 D). The initially recognizable lamellapodia and filopodia were no longer obvious.

We also tested the responses of cortical neurons to eprhin-A5 incubation in a culture setting. Similarly, the elaborate growth cones of the cortical neurons also collapsed shortly after ephrin stimulation (data not shown).

Since immunoprecipitation and immunostaining methods both indicated that Nck1 interacts with phosphorylated EphA3 receptor in cultured neurons, we were interested in determining if Nck1 was critical to the process of EphA3 mediated growth cone collapse. By expressing dominant negative Nck1 clones in hippocampal neurons, we examined whether the disruption of endogenous Nck1 functions blocked ephrin-A5 induced growth collapse. 


\section{Construction of adenoviruses carrying wild type and dominant negative clones of}

\section{Nck1}

Neurons are difficult to transfect because they are sensitive to harsh experimental procedures. Regular lipid-based transfection reagents such as Lipofectamine do not produce high efficiency $(<5 \%)$ and neurons are sensitive to the toxicity of these reagents. Calcium phosphate method does not have high toxicity but the efficiency is also very low $(<10 \%)$. Nucleofection, a method based on electroporation principles, has decent efficiency $(>20 \%)$ but requires a large number of cells $\left(>10^{6}\right.$ cells) for each experiment. After evaluating these methods, we recognized that adenovirus-mediated gene transfer was the ideal approach. Adenovirus is known to infect neurons with high efficiency and the expression of desired genes is not dependent on cell division. The latter attribute is especially meaningful since cultured neurons do not proliferate. In addition, adenoviruses are not cytotoxic to neurons so the infected neurons stay relatively healthy.

The procedure of adenovirus construct generation was summarized [Fig. 4-5, (He et al. 1998)] and described in detail in Chapter VI. Recombinant adenoviral vectors, pAdEasy-CMV, pAdEasy-CMV-Nck1WT, pAdEasy-CMV-Nck1R308K and pAdEasyCMV-Nck1W38/143/229K were each digested and transfected into HEK293A cells to generate viruses. On these plasmids, the inserted gene of interest and an EGFP gene were located behind separate CMV promoters, allowing easy recognition of the infected cells. The transfected 293A cells were harvested seven days later and the adenoviruses were collected. Several rounds of amplification were carried out to obtain high-titer viral stocks. The final adenovirus stocks were used to infect 293A cells and the expression of 
Nck1 mutants in infected cells was confirmed by Western blot (Fig. 4-6 A). In general, for neuronal infection, recombinant adenoviruses with a titer of $10^{9}$ to $10^{10} / \mathrm{ml}$ of recombinant adenovirus were used.

\section{Nck1 DN mutants do not prevent growth cone collapse induced by ephrin-A5}

To examine effects of Nck1 DN mutants on the growth cone collapse, E18 rat embryos were sacrificed and the hippocampi were collected. In a glass dish, the hippocampi were cut into small explants of uniform size. These hippocampal explants were plated in serum free neurobasal medium on glass culture dishes that had been coated with Poly-D-lysine and Laminin.

Adenovirus stocks were diluted in neurobasal medium and filtered with a .25 um filter to remove all residual cell debris. The adenovirus solution was added to the hippocampal explant cultures. After overnight incubation, the adenoviruses were removed and fresh culture medium was added.

On day 4 , the cultured explants were treated with or without cross-linked ephrin-A5 for 30 minutes before being fixed with fresh fixative. The explants were permeablized with TritonX-100 and then stained with Texas-red phallodin to visualize the actin cytoskeleton, allowing easy observation of growth cones.

The infected neurons were identified by monitoring EGFP expression. On each explant, every green growth cone that was on the periphery of the explant was examined and marked as either "collapsed" or "uncollapsed". The number of all infected growth cones $(\mathrm{G} 1)$ and the number of all infected growth cones that had collapsed $(\mathrm{G} 2)$ were counted and the growth cone collapse index was expressed as $I=G 2 / G 1$. For each 
experiment, at least 5 explants were quantified and the average I value was calculated. The results were found to be consistent in three experiments and one representative experiment is shown in Figure 4-6 B.

Before ligand stimulation, about $33 \%(+/-4 \%)$ of wild type hippocampal growth cones expressing only EGFP were collapsed. After 30 minutes of ephrin-A5 treatment, about $77 \%(+/-2 \%)$ of all EGFP expressing growth cones were collapsed. When explants expressing various Nck1 mutants were inspected, we found that the expression of any Nck1 mutant did not alter the response of hippocampal neurons to ephrin-A5 stimulation. Despite the expression of dominant negative Nck1 clones, the majority of infected growth cones still collapsed in reaction to ephrin-A5, similar to the EGFP control. It was therefore concluded that the expression of Nck1 mutants through adenoviruses was not able to disrupt EphA3 mediated growth cone collapse induced by ephrin-A5 treatment.

\section{Discussion}

Nck1 binds to activated EphA3 receptor in neurons and they co-localize highly on the growth cones

During development, many neural circuits form and many signaling factors are involved in this process, among which is the EphA3 receptor. For instance, interactions of EphA3 with the ephrin-A2 and -A5 ligands on the tectum is the shaping force for a precise retinotectal topographic projection (Cheng et al. 1995; Drescher et al. 1995). In the past two decades, much effort has been invested in understanding how the downstream signaling pathways are regulated. 
We isolated Nck1 in a screen for EphA3 binding proteins in an embryonic brain cDNA library. In HEK293A cells, we have found that Nck1 SH2 domain binds directly to the tyrosine residue 602 of EphA3. Additionally, in 293A cells, the expression of dysfunctional Nck1 proteins is able to lead to disrupted EphA3 signaling. Furthermore, at E18, when EphA3 expression is present in cortical and hippocampal neurons, Nck1 is also co-expressed These data together strongly suggest Nck1 plays a major part in EphA3 signal transduction in neurons.

In contrast to control IgG, Nck1 specific antibody successfully precipitates EphA3 molecules. This co-precipitation of EphA3 and Nck1 was dependent on the activation of EphA3 receptor by ephrin-A5 treatment. The necessity of ligand treatment for EphA3Nck1 binding is in accordance with previous research and indicates that phosphorylation and activation of EphA3 receptor are paramount to Nck1 binding.

Immunostaining, on the other hand, helped us to determine the sub-cellular localization of EphA3 receptor and Nck1 molecule. It was found that EphA3 molecules were localized on the plasma membrane of both the cell body and the neurites, especially on the growth cones. This finding was consistent with the fact that growth cones guide axons in a complex environment towards their appropriate targets. Immunostaining also revealed that after ephrin-A5 treatment, Nck1 molecules translocated from the cytosol to interact with activated EphA3 receptors. Consequently, both proteins were found to have a high degree of co-localization on the plasma membrane.

The data from neuron cultures and from transfected HEK293A cells support our hypothesis that Nck1 plays a significant role in EphA signal transduction. 


\section{Nck1 signaling may regulate aspects of EphA3 signaling but not growth cone}

\section{collapse}

EphA3 is expressed in the hippocampi in a graded manner (medial high, lateral low) along with EphA4, -A5 and -A7. The corresponding ligands ephrin-A2, -A3, -A5 are expressed in the septum in a ventral high, dorsal low gradient (Gao et al. 1996; Zhang et al. 1996). During the formation of this projection, the growth cones of medial hippocampal neurons are turned away from the ventral lateral septum due to the high expression of EphA3 receptors, which results in strong repulsion (Gao et al. 1996; Zhou 1997; Otal et al. 2006). Consequently, the medial axons retract and move towards the dorsal lateral part of the septum, where ephrin-A ligands are expressed at a lower level. The lateral hippocampal neurons, on the other hand, express low level of EphA receptors and are able to project into the ventral septum. Therefore, the complementary gradients of receptor and ligands enable hippocampal neurons to innervate appropriate targets on the septum and form a precise topographic projection.

Similarly, neurons in culture exhibit growth cone collapse and axon retraction, in reaction to ligand addition to the culture medium. This observation provides the foundation of growth cone collapse assay. However, as we noted, some growth cones remained uncollapsed after ephrin treatment probably because a heterogeneous population of hippocampal neurons was tested. Since the lateral hippocampal neurons express low levels of Eph receptors, they are less responsive to ephrin-A5 treatment.

Using adenoviruses expressing different genes, we tested our hypothesis that Nck1 is involved in EphA3 signaling in hippocampal growth cone collapse. Surprisingly, no alteration to the growth cone collapse rate of was detected in infected hippocampal 
neurons, in which the function of endogenous Nck1 was blocked by dominant negative mutants.

Although we tested the infection of various mutants in 293A cells by Western blot, we did not confirm the expression of these genes in neurons. In addition, we relied on EGFP expression to help us identify infected neurons, but the presence of green fluorescence in the cells does not necessarily reflect the expression of the desired genes at a high level or alternately, the infection efficiency and expression level in neurons were not high enough in our assay. It is expected that a significant amount of dysfunctional Nck1 molecules is required to override the function of endogenous Nck1, which was very abundant in neurons (Fig. 4-1 B). Therefore, other methods of blocking Nck1 function should be tested before we conclude that Nck1 is not involved in the process of growth cone collapse triggered by ephrin-A5 stimulation. For instance, siRNA is a commonly used technique for knocking down the endogenous expression of the protein of interest. By using Nck1 specific siRNA in neurons to effectively disrupt Nck1 translation, we would be able to evaluate Nck1's role through a different approach.

We use hippocampal explants in our assay because the hippocampal neurons express both EphA3 and Nck1 and are well characterized. However, the graded expression of multiple Eph receptors across the hippocampus suggests a high degree of redundancy (Gao et al. 1999). When dysfunctional Nck1 genes were expressed in these neurons, it is probable that the signaling through Eph receptors was not completely silenced, therefore leading to no detectable alteration to growth cone collapse.

When the only Nck homolog in Drosophila Dock was eliminated, severe disruption of the correct innervations of photoreceptor neurons was observed (Garrity et al. 1996; 
Rao and Zipursky 1998). These neurons, instead of stopping at their proper targets, tend to overshoot. Furthermore, the expression of Ste20-like kinase Misshapen (Msn, a homolog of mammalian NIK) was found to co-localize with Dock in the growth cones of these neurons ((Ruan et al. 1999; Su et al. 2000). Loss of Msn resulted in a similar phenotype to the R cells as in Dock knockout. These combined observations suggest that Nck proteins, which are conserved in mammalians are likely to regulate growth cone collapse as well. Since the two mammalian Nck members, Nck1 and Nck2 show high homology $(60 \%)$ and their binding preferences seem to be indistinguishable from each other (Frese et al. 2006), it is very likely that the endogenous expression of Nck2 compensates for the loss of normal Nck1 function in hippocampal neurons. Even though we did not test the expression of Nck2 in neurons, it is very likely that Nck2 is also present in hippocampal and cortical neurons. We suspect that this redundancy explains why the expression of dominant negative Nck1 was not able to lead to a reduced rate of growth cone collapse, similar to how the Nck1 DN genes were not able to completely reverse ephrin-A5 induced phenotypes in 293A cells expressing EphA3. Previously, when Nck1, Nck2 double knockout mice were generated, it was found out that the double knockout animals died during embryonic development. An inspection of fibroblasts of these animals reveals that these cells become defective in cell motility and actin restructuring (Bladt et al. 2003). An analysis of the hippocampal axon guidance in Nck1, Nck2 double knockout animals would therefore shed light on the possible redundant role of Nck2 in the EphA3-Nck1 signaling pathway.

Another possibility is that though our hypothesis about Nck1's involvement in EphA3 mediated signaling still holds true, Nck1 does not directly regulate growth cone 
collapse, one aspect of EphA3 signaling in neurons. As we discussed earlier, ephrin-A5 treatment of EphA3 expressing neurons causes an immediate collapse of growth cones in the majority of the neurons. This is followed by a period during which neurite growth is generally stalled. Later, the neurites may exhibit either retraction or turning as they are further repelled by the ligands (Muller et al. 1990; Weinl et al. 2003; Huot 2004). These observations show that the response to ephrin treatment takes place in multiple stages and probably requires the coordination of many signaling pathways, especially the cytoskeleton machinery. For instance, the actin network in the growth cone and the microtubule filaments in the neurite shaft both have to be mobilized during this process. Nck1 has been known to link receptor tyrosine kinase to downstream signaling pathways such as the p21 cdc42/Rac activated kinase (PAKs), the WASP mediated actin restructuring and Sos/Ras/MAPK pathways (Tang et al. 1997). Through its many effectors, Nck1 is likely to be an integral part of EphA3 signaling in developing nervous system. Since our assay focused on detecting a difference in growth cone collapse on the hippocampal neurons but not other changes, more experiments need to be designed to assess if Nck1 plays a part in other aspects of ephrin-A5 induced responses. For instance, neurite retraction assay is an assay that has been used in some labs to test the signaling of Eph receptors in neurons. When young dissociated hippocampal neuron culture are treated with ephrin-A5 overnight, a $\sim 50 \%$ of average neurite retraction was noted (Dr. S. Hsu and Dr. S. Wang, personal communication). It will be intriguing to find out when the function of Nck1 is compromised, whether the hippocampal neurons still retract the axons at such a rate. 

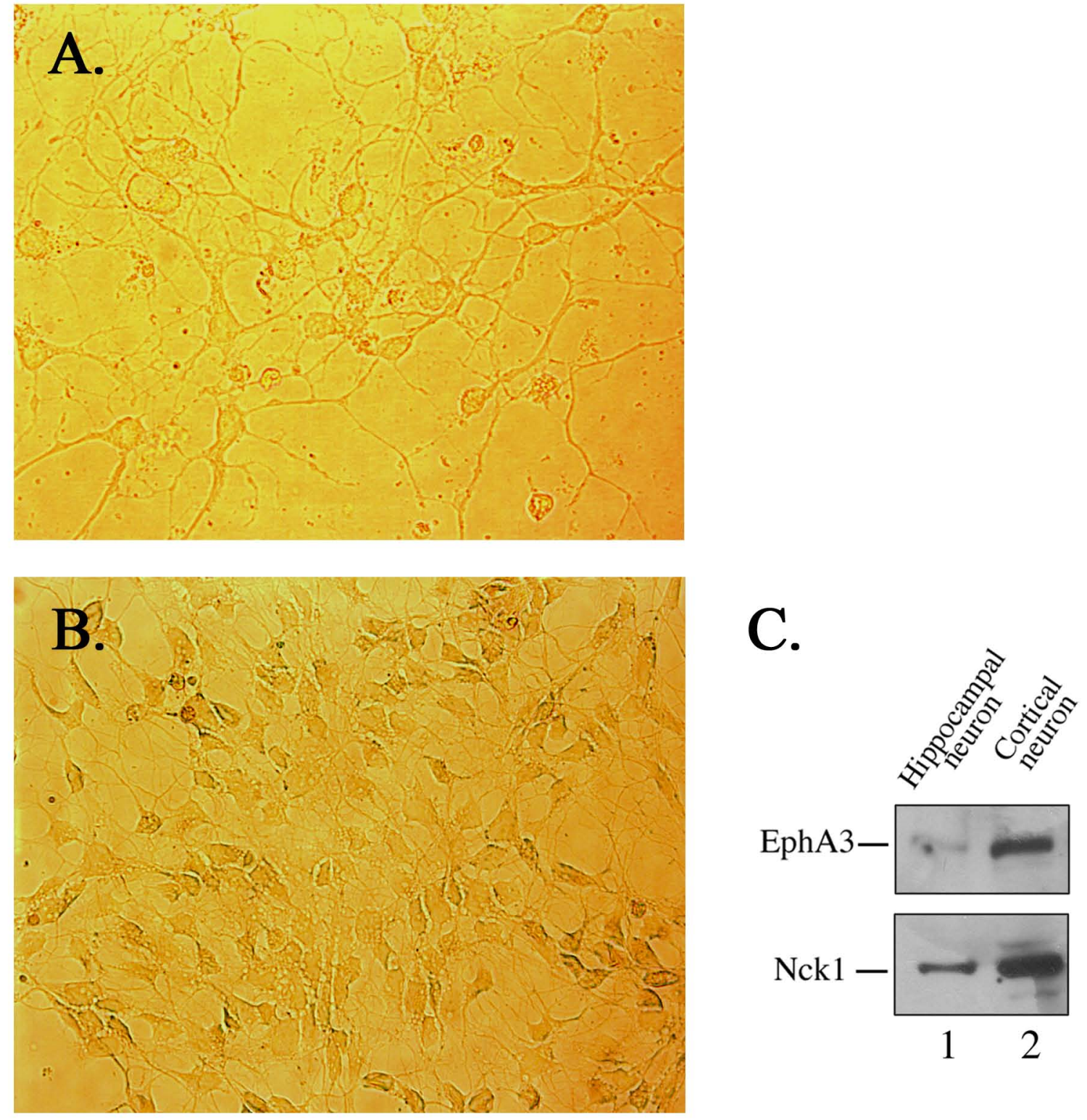
Figure 4-1. Expression of EphA3 and Nck1 in cortical and hippocampal neuron cultures.

(A) Bright field image of E18 rat embryonic hippocampal neuron culture. (B) Bright field image of E18 rat embryonic cortical neuron culture. (C) The endogenous expression of EphA3 and Nck1 in neuron cultures, as assessed by Western blot. 

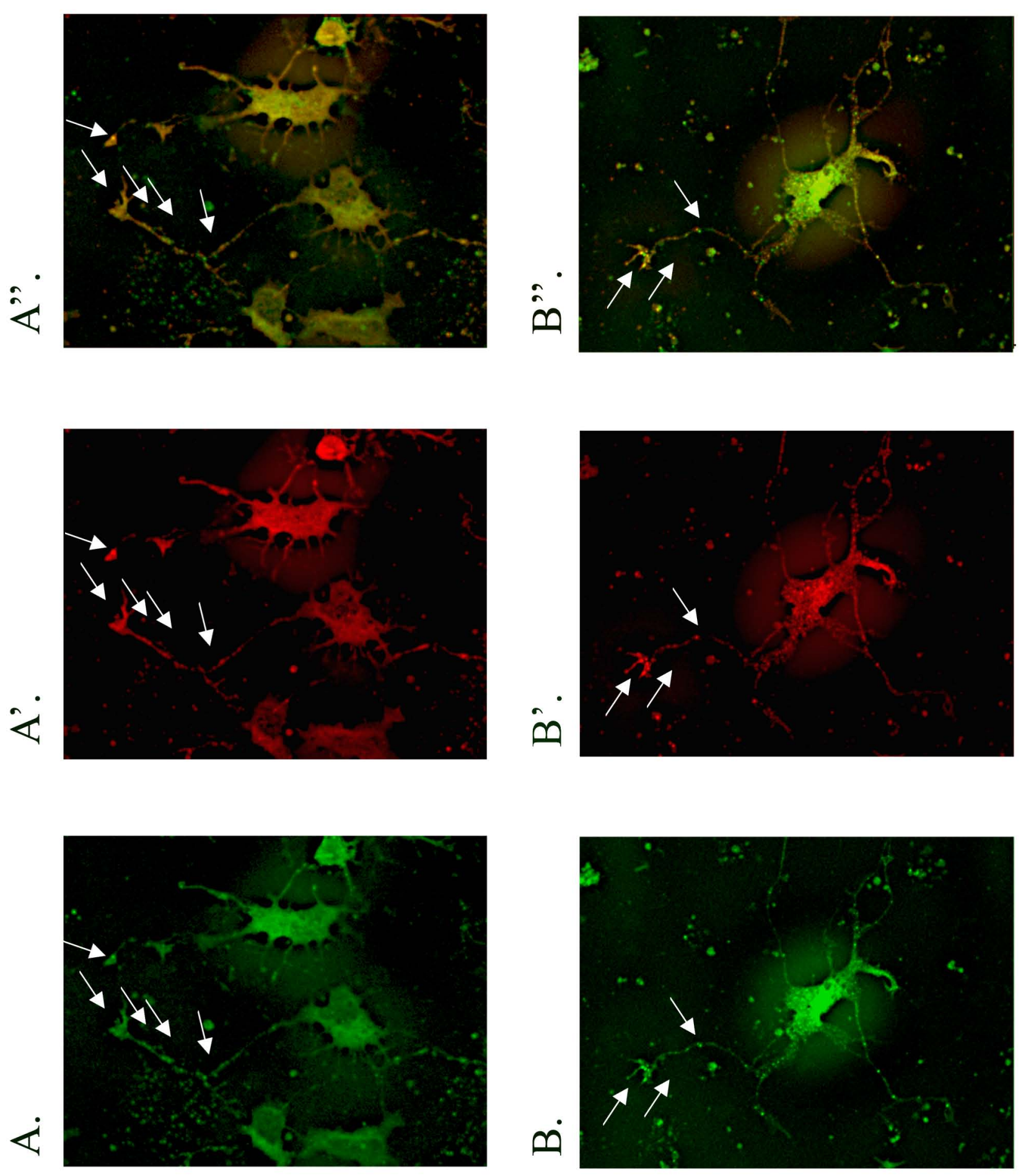
Figure 4-2. Co-localization of EphA3 and Nck1 in hippocampal neuron growth cones.

E18 rat embryonic hippocampal neurons were cultured for two days before being treated, fixed, and stained. Anti-EphA3 antibody followed by FITC secondary antibody was used to stain for EphA3. Anti-Nck1 antibody with Cy3 secondary antibody was used to stain Nck1. Two groups of cells are shown in A-A"' and B-B"' respectively. Panel A'" and B"' are superimposed pictures of two single channels and the white arrows indicate colocalization of EphA3 and Nck1. 


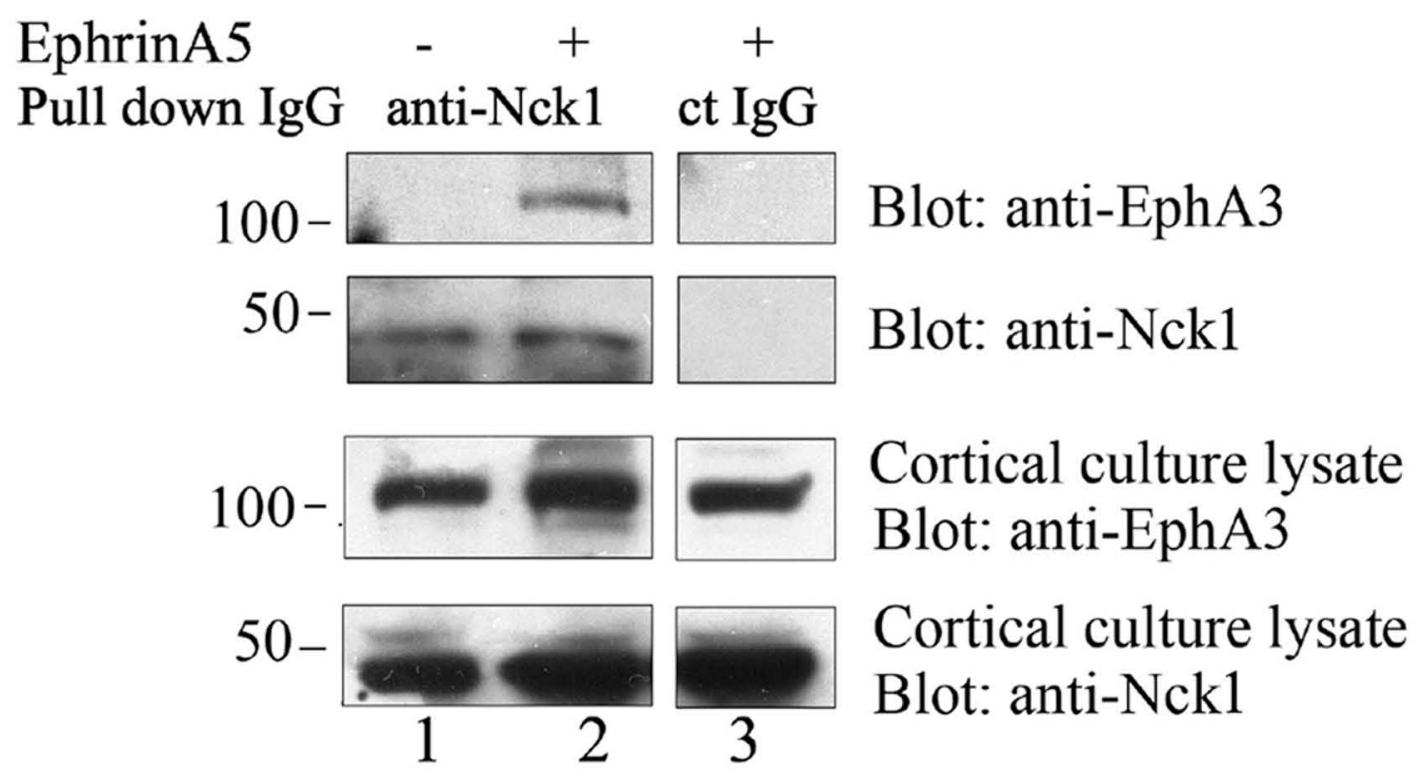


Figure 4-3. Co-precipitation of EphA3 and Nck1 in cortical neurons.

EphA3 molecules were precipitated from E18 rat hippocampal neuron lysates by Nck1 specific antibody in a stimulation-dependent manner. Total expression of EphA3 and Nck1 in the neuron lysates is also shown. 

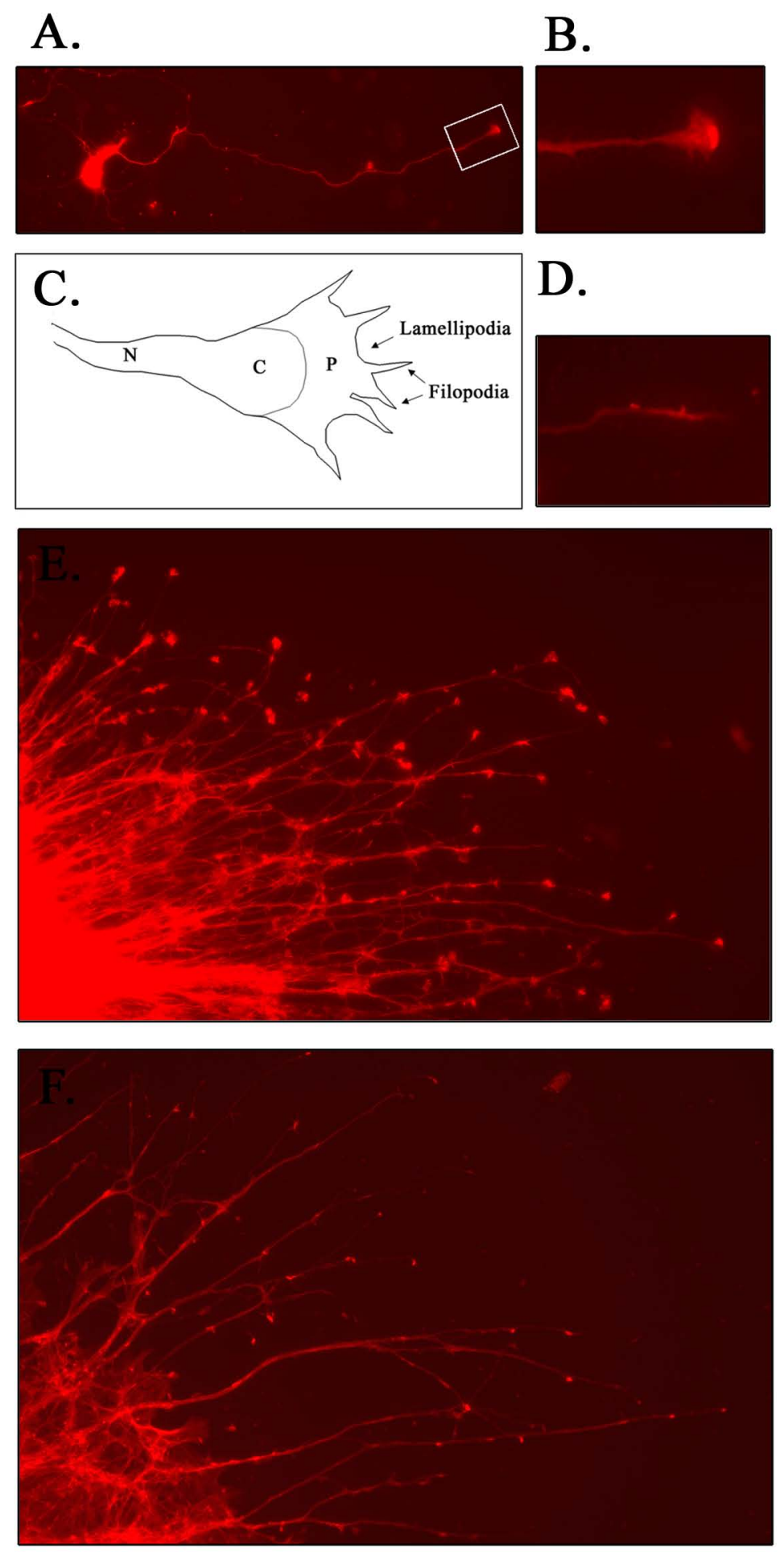
Figure 4-4. Growth cone collapse induced by ephrin-A5 treatment in hippocampal neurons.

(A). Fluorescent image of cultured hippocampal neurons after staining with Texas-red Phallodin. (B) Enlarged box in panel A. (C) A schematic representation of a representative growth cone. "N" denotes the neurite; "C" indicates the central domain of a growth cone and "P" marks the peripheral domain that includes the lamellapodia and filopodia. (D). A collapsed growth cone after ephrin-A5 treatment. (E). Hippcoampal explant cultures displaying many growth cones after two days. (F). A brief treatment with ephrin-A5 resulting in the collapse of growth cones in the explant culture. 

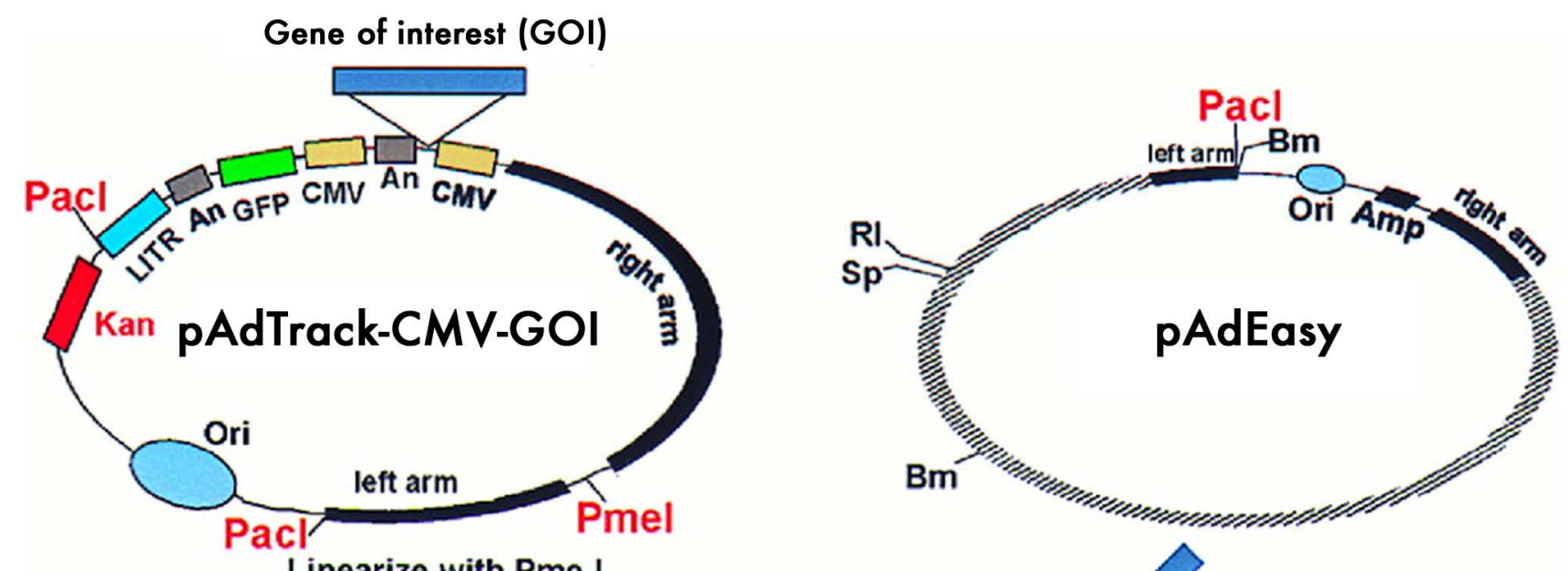

Linearize with Pme I
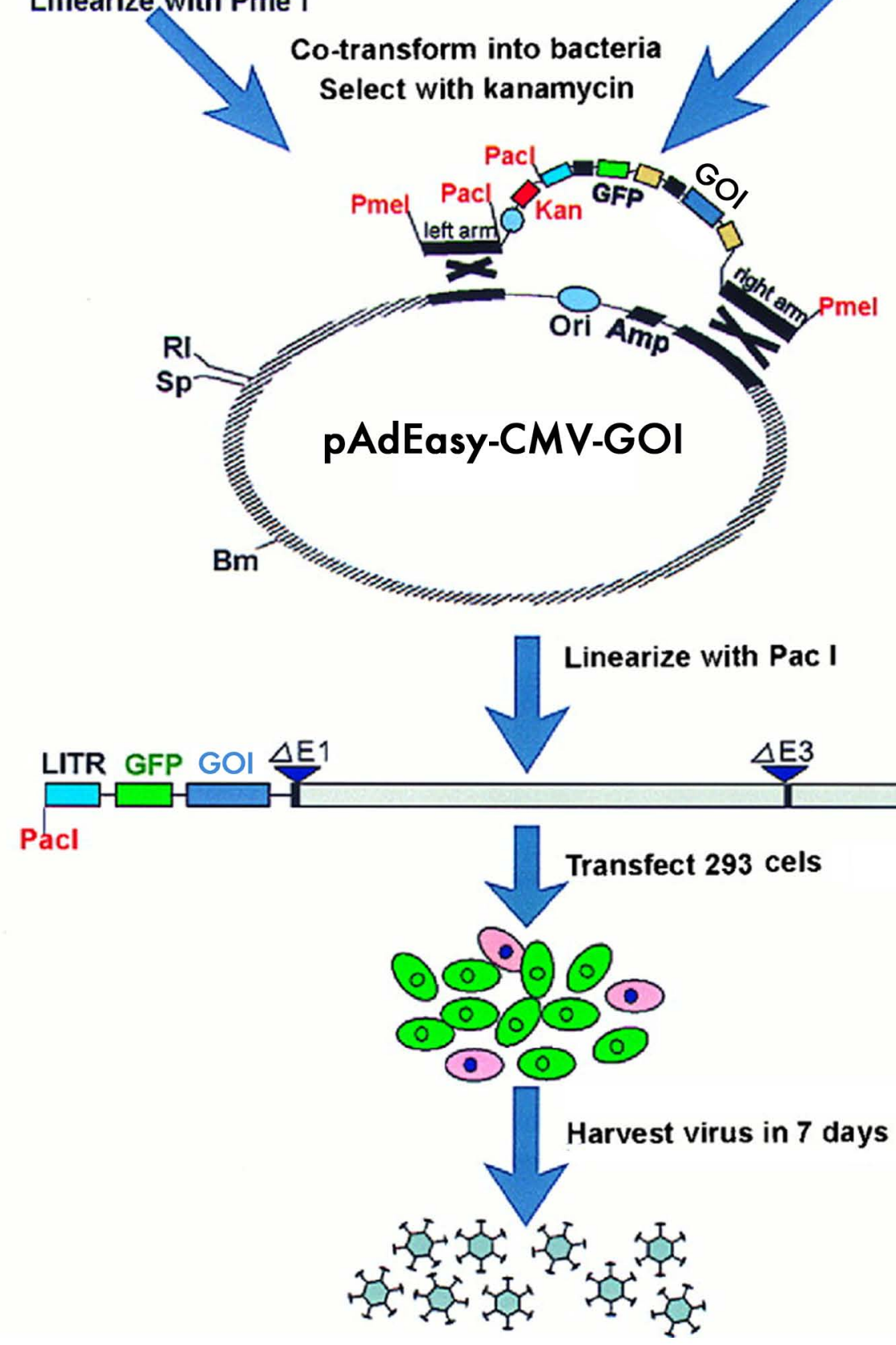
Figure 4-5. A schematic outline of the construction of adenoviral vectors carrying the gene of interest.

The figure is modified from the paper $A$ simplified system for generating recombinant adenoviruses (He et al. PNAS, 1998). The gene of interest was cloned into pAdTrackCMV vector and the resulted plasmids were recombined with pAdEAsy vectors in the $E$. coli strain BJ5183 to obtain desired recombinant plasmids. These plasmids were then digested and transfected into 293 cells to produce adenoviruses. In the plasmid maps, the "left arm" and "right arm" are the regions mediating homologous recombination between pAdTrack-CMV-GOI and pAdEAsy. Abbreviations: An, polyadenylation site; Bm, BamHI; RI, EcoRI; LITR, left-hand ITR and packaging signal; RITR, right-hand ITR; Sp, SpeI. 
A.
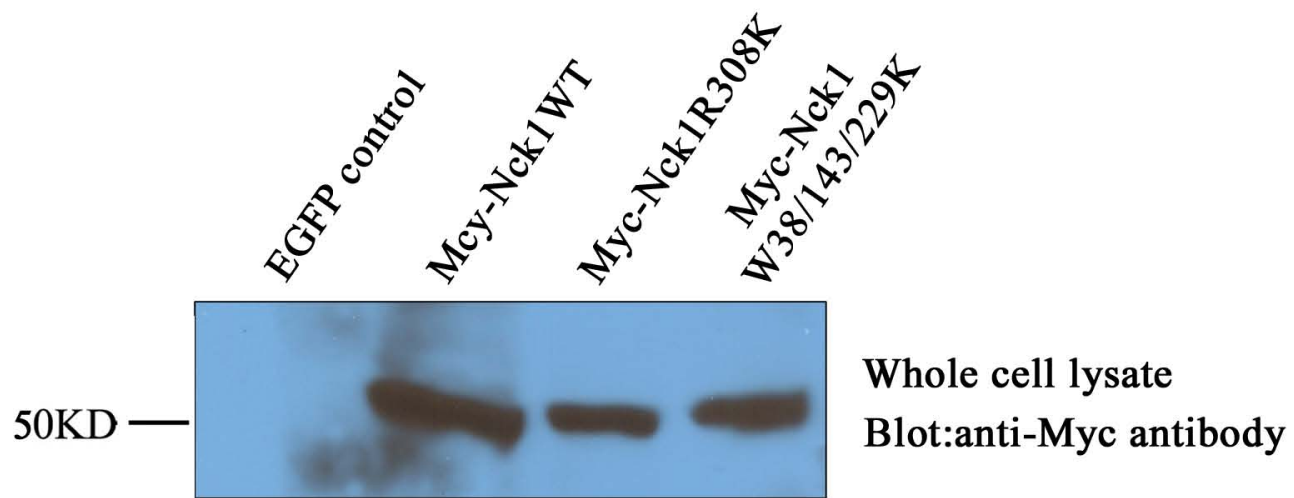

B.

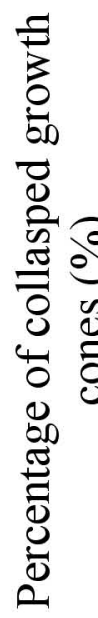

90.0
80.0
70.0
60.0
50.0
40.0
30.0
20.0
10.0
0.0
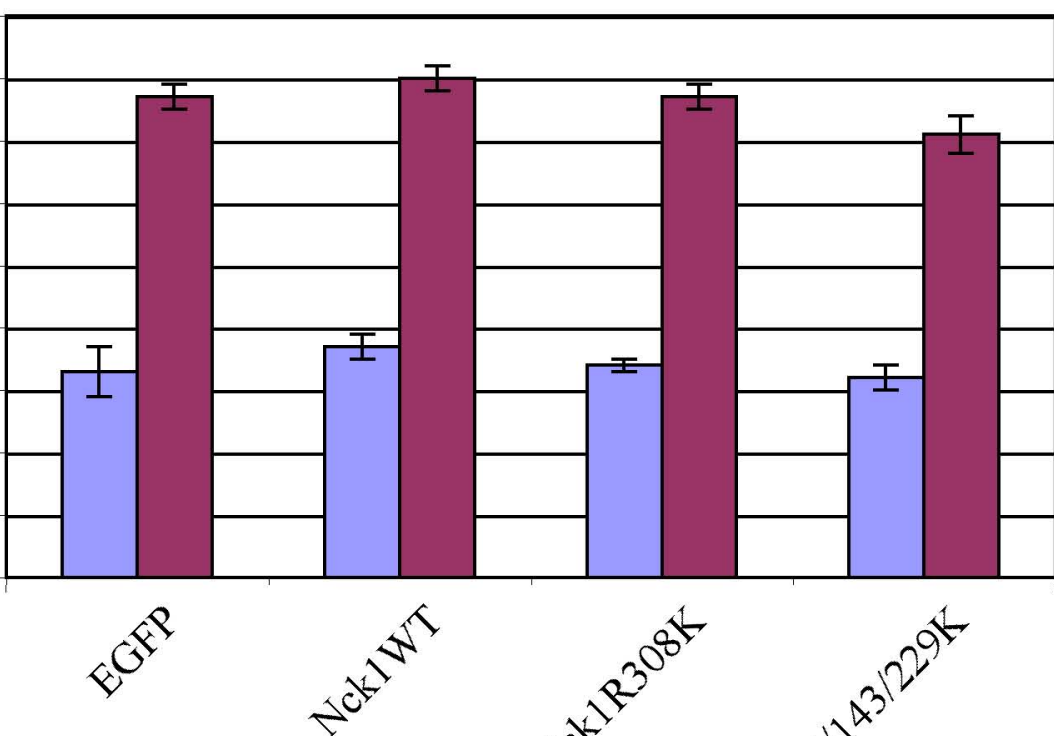

$\square$ no ephrin-A5

$\square$ ephrin-A5

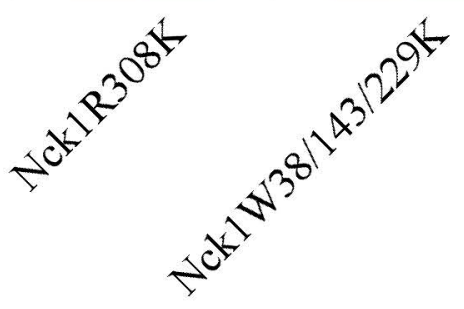


Figure 4-6. Growth cone collapse assay on neurons infected with adenoviruses expressing Nck1WT, Nck1R308K, and Nck1W38/143/229K.

(A) The expression of various Nck1 mutants through adenovirus infection was confirmed by Western blot. (B) When the amplified adenoviruses were used to infect hippocampal neuron explants, no significant differences in growth cone collapse response was observed in neurons treated with ephrin-A5. 


\section{Chapter V. Assessment of the binding of EphA3 with Grb2 and $\underline{\text { RasGAP }}$}

\section{Introduction}

We have shown in earlier chapters that Nck1, consistent with the yeast two-hybrid screen results, is able to regulate key aspects of EphA3 signaling. Furthermore, we showed that the interaction between Nck1 and Eph3 occurs through the SH2 domain and tyrosine 602 , respectively. Tyrosine residue 602 is located in the juxtamembrane domain of EphA3 and is highly conserved throughout the Eph receptor family.

Even though Nck1 is the only SH2 domain containing protein identified in the yeast two-hybrid screen, it is well-known that other $\mathrm{SH} 2$ domain containing proteins also initiate direct interactions with the Eph receptors ((Birge et al. 1996; Mellitzer et al. 2000; Nakamoto 2000; Buday et al. 2002). Intriguingly, many of these proteins have been found to interact with the juxtamembrane domain of the Eph receptors (Schmucker and Zipursky 2001; Klein 2004).

Thus, a question arises as to whether Nck1 and other $\mathrm{SH} 2 / \mathrm{SH} 3$ domain containing proteins may compete for the same binding sites once EphA3 receptor is activated. To answer this question, we attempted to map the binding locations on EphA3 with Grb2 and RasGAP. Both of these proteins play key roles in the regulation of Ras/MAPK pathway, and it has been known the activity of MAPK pathway is closely correlated with cell behavior like neurite retraction and growth cone collapse (Tong et al, 2003). 
Characterization of EphA3 interaction with these proteins would therefore shed light on how SH2 domains of various proteins compete for binding and induce varied phenotypes.

\section{Results}

\section{The SH2 domain of both Grb2 and RasGAP bind strongly to EphA3}

Grb2 is a small adaptor protein that contains one SH2 domain and two SH3 domains (Fig. 5-1 A). Numerous studies have shown that Grb2 physically interacts with EphA2, EphB1 and EphB2 (Stein et al. 1996; Pratt and Kinch 2002; Moeller et al. 2006). Grb2 is known to bind to Sos, a RasGEF through its SH3 domains (Egan et al. 1993; Buday et al. 1994). When Grb2/Sos complex associates with phosphorylated RTKs, Sos is activated and catalyzes the replacement of GDP with GTP on Ras. Active Ras is then able to upregulate MAPK activities (Zarich et al. 2000; Avruch et al. 2001).

RasGAP is a $120 \mathrm{KD}$ cytosolic protein that promotes the conversion of GTP to GDP on Ras small GTPases. As a result, Ras is inactivated and the MAPK activity is downregulated (Elowe et al. 2001; Tong et al. 2003). RasGAP consists of two SH2 domains, one $\mathrm{SH} 3$ domain, one pleckstrin homology domain $(\mathrm{PH})$, one protein kinase $\mathrm{C} 2$ domain (C2) and one Ras guanine activating protein domain (Ras-GAP) (Fig. 5-1 A). Previous reports have shown that RasGAP associates with EphB2, forming a large protein complex that includes RasGAP, Nck1, and EphB2 (Holland et al. 1997; Tong et al. 2003). These studies also show that the binding occurs mostly through the N-terminal SH2 domain of RasGAP.

The SH2 domain of Grb2 and the N-terminal SH2 domain of RasGAP were each fused to GST peptide in pGEX 4T-2 vectors. Beads conjugated with GST fusion proteins 
were used in pulldown experiments. The precipitated proteins were analyzed by Western blot (Fig. 5-1B). The analysis shows that GST-Grb2SH2 is able to precipitate phosphorylated EphA3 receptor, indicating that Grb2 protein interacts with the receptor. We also confirmed the association of RasGAP with EphA3 through the N-terminal SH2 domain (Fig. 5-1 B). The abundance of precipitated EphA3 in both lane 4 and lane 5 indicates that the binding between EphA3 with Grb2 or RasGAP is strong.

\section{Tyrosine 602 on EphA3 is a binding site for Grb2 SH2 domain}

After validating the strong binding of EphA3 and Grb2, we conducted another pulldown experiment to identify the binding location of Grb2 on EphA3.

In summary, the tyrosine mutants (Y596F, Y602F, Y779F, Y937F, Y596E, Y602E), EphA3WT and K653R were overexpressed in 293A cells. The cells were treated with ephrin-A5, lysed, and incubated with sepharose beads conjugated to GST-Grb2SH2 proteins. The precipitated proteins were analyzed by Western blot (Fig. 5-2).

The Western blot reveals the binding of Grb2 and EphA3 is dependent on the kinase activity of EphA3 since the kinase dead mutant K653R doesn't show any binding with Grb2 SH2 domain. Among the tyrosine mutants, the Y779F and Y937F mutants both exhibit normal binding with Grb2, while both Y596F and Y602F display no interaction with Grb2, similar to K653R. When the Y596 and Y602 were replaced by glutamate, disparate results were obtained. While Y596E mutant can be precipitated by Grb2 SH2 domain at a normal level, a significantly lower amount of Y602E is precipitated by Grb2 protein (Fig. 5-2). This result was consistent in three separate experiments. Upon 
examining the phosphorylation pattern of these mutants (Fig. 3-3A), we conclude that the tyrosine 602 is one of several major binding sites of Grb2 SH2 domain.

\section{RasGAP binds to EphA3 partially through tyrosine 602}

When GST-RasGAPNSH2 was incubated with cell lysates containing various EphA3 mutants, similar results were found (Fig. 5-3). Both Y596F and Y602F are not precipitated by RasGAP N-terminal SH2 domain, while other tyrosine to phenylalanine mutants bind to RasGAP N-terminal SH2 domain normally. When the two tyrosine to glutamic acid mutants were tested, we found that Y596E binds normally to RasGAP, while Y602E exhibits only partial binding. This finding suggests that the Y602 in the juxtamembrane domain also mediates binding with the RasGAP N-terminal SH2 domain but other unknown binding sites also exist.

\section{Discussion}

\section{EphA3 interacts with multiple SH2 domain containing proteins}

A multitude of proteins that have been found to bind to Eph receptors contain $\mathrm{SH} 2$ domains (http://proteoscape.uchicago.edu/sh2/index.html, Fig. 5-4). Among them are well known adaptor proteins such as Crk, Grb2, Grb10, Nck1, SHC, and SLAP (Pandey et al. 1995; Stein et al. 1996; Holland et al. 1997; Stein et al. 1998; Lawrenson et al. 2002; Pratt and Kinch 2002). These adaptors do not possess catalytic abilities, but contain multiple protein binding domains (Fig. 5-4 A). They serve as scaffold proteins that not only link activated tyrosine receptors to downstream effectors, but also bring downstream effector proteins in close proximity with each others. 
Src family kinase (SFK) members are also known to bind to Eph receptors and they include Fyn, Yes, Src, and Abl/Arg (Ellis et al. 1996; Choi et al. 1999; Yu et al. 2001). These proteins possess similar domain structure and catalyze phosphorylation of cytoplasmic substrates (Fig. 5-4 B). For instance, Src kinase phosphorylate R-Ras and reduces cell adhesion by regulating integrin signaling (Zou et al. 2002). Interestingly, it has been shown that EphB2 has the ability to phosphorylate R-Ras as well (Zou et al. 1999).

Another group of SH2 containing proteins are enzymes that are not SFK members. This group of proteins includes p85 PI3 kinase, PLC gamma, RasGAP, SHEP-1, and SHP2 (Holland et al. 1997; Dodelet et al. 1999; Boyd and Lackmann 2001). These proteins (Fig. 5-4 C) are able to play direct roles in regulating signaling pathways. For example, SHEP1 possesses one SH2 domain and one Ras-GEF domain. It binds to R-Ras and Rap1A through the Ras-GEF domain and brings these small GTPases close to EphB2 receptor juxtamembrane domain (Dodelet et al. 1999). Once the R-Ras molecules are located close to the receptor/effector complex near the plasma membrane, they become phosphorylated by the Src kinase or EphB2, as mentioned earlier.

Of special interest to us is the capability of these binding proteins to form complexes among themselves. Grb2 has been found to bind to Crk, SHC, Abl/Arg, PLC gamma, PI3 kinase, SHP2, and RasGAP (Saleem et al. 1995; Wang and Moran 1996; Wong and Johnson 1996; Pei et al. 1997; Riordan et al. 2000; Lamprecht et al. 2002; Plattner et al. 2004). These multilateral interactions may enhance the association with Eph receptors and give rise to a tightly organized signaling complex through which the receptor signals efficiently. 


\section{Grb2 may compete with Nck1 for the binding of EphA3}

Using the power of the GST pulldown assay, we confirmed that Grb2 is able to bind phosphorylated EphA3. The characterization of EphA3-Grb2 interaction shows that it is different from the binding of Grb2 with another EphA receptor, EphA2. As reported earlier, Grb2 interacts with activated EphA2 indirectly through another SH2 containing protein, SHC (Pratt and Kinch 2002).

Furthermore, the GST pulldown assays demonstrate that tyrosine 602 in the juxtamembrane domain is one binding site for the Grb2 $\mathrm{SH} 2$ association. When this tyrosine residue is mutated to a phenylalanine residue, Grb2 fails to bind EphA3. Replacement at this position with glutamic acid rescues binding. Tyrosine 596, in comparison, modulates the kinase activity of the receptor, but does not serve as a direct binding site. In addition, mutation of tyrosine 779 and 937 did not result in loss of binding with EphA3, suggesting that these residues play an unimportant role in mediating the interaction with Grb2 SH2 domain. It is important to point out that other binding sites also exist since Y602E displays some binding with Grb2. A more comprehensive experiment needs to be performed in order to identify the rest of the interacting tyrosine residues.

Since Grb2 and Nck1 share one identical binding site, they are likely to compete for binding when they are both present in EphA3 expressing cells. Nck1, through competition with Grb2, could compromise the Sos/Ras/MAPK pathway and lead to down-regulated MAPK activity. However, because Grb2 is able to bind to multiple 
locations and also indirectly through other proteins, the effect of this competition is not known at present but it would be interesting to find out.

\section{RasGAP binds to EphA3 through multiples sites including tyrosine 602}

Our experiment data shows that p120RasGAP also binds to tyrosine 602 in the juxtamembrane domain, while tyrosine 596 does not play a part in the interaction with RasGAP. However, tyrosine 602 is only responsible for mediating part of the interaction, as evidenced by the mutation of residue 602 to glutamate, which did not entirely abrogate binding with RasGAP. It can be inferred that other binding sites for RasGAP exist on EphA3. Similar to our finding, Elowe et al have reported that mutation of tyrosine 604

and 610 residues in EphB2 (equivalent to 596 and 602 of EphA3) to glutamates, results in a reduction rather than complete loss of binding with RasGAP (Elowe et al. 2001). However, a different group reported that when both the juxtamembrane domain tyrosines and tyrosine 667 were mutated on EphB2, a complete loss of binding with RasGAP occurred (Tong et al. 2003). Since tyrosine 659 of EphA3 is equivalent to tyrosine 667 of EphB2, it is probable that tyrosine 659 in the kinase domain is the other binding site that mediates the recruitment of RasGAP to the activated EphA3. More research has to be conducted in order to test this hypothesis.

In the current study, we only tested the binding of N-terminal SH2 domain of RasGAP. Even though previous studies show that the C-terminal SH2 domain usually does not interact with activated tyrosine kinase receptors (Holland et al. 1997), there exists a possibility that this domain mediates synergistic binding with $\mathrm{N}$-terminal $\mathrm{SH} 2$ domain. Furthermore, indirect binding through Crk and Nck1 has been reported. 
Therefore, even though tyrosine 602 is a common site for both Nck1 and RasGAP to bind, the two proteins may be capable of multilateral binding, similar to the association of RasGAP and Nck1 with EphB2 (Holland et al. 1997). Furthermore, it has been shown that p62dok assists the association of RasGAP and Nck1 since both proteins bind to p62dok through their SH2 domains (Tang et al. 1997).

As we discussed earlier, RasGAP directly regulates the activity of Ras and consequently, reduces both integrin signaling and ERK activities, critical to Eph receptor signaling. In NG108 cells, activated EphB2 recruits RasGAP and down-regulates MAPK activity (Elowe et al. 2001; Tong et al. 2003), leading to neurite retraction and growth cone collapse. Grb2 on the other hand, activates Ras through Sos, and up-regulates ERK activities. Since our data suggest that both proteins bind to EphA3 through tyrosine residue 602 at least partially, the recruitment of Grb2 or RasGAP onto activated EphA3 may provide an explanation for different responses induced ephrin treatment.

\section{Other SH2 domain proteins that interact with the juxtamembrane domain}

Our studies show that Nck1, Grb2 and RasGAP all bind to EphA3 receptor via interaction with tyrosine 602 in the juxtamembrane domain (Fig. 5-5). In addition, previous studies have shown that the juxtamembrane domain is very important in the interaction with many SH2 domain containing proteins such as Fyn, Yes, Src, Crk, SLAP, SHEP-1, PLC gamma, and Abl/Arg kinase (Ellis et al. 1996; Stein et al. 1998; Zisch et al. 2000; Yu et al. 2001). Therefore, we speculate that the preferential association of different sets of $\mathrm{SH} 2$ containing proteins with Eph receptor is likely to 
translate to context-dependent responses to ephrin stimulation. More research is needed in order to understand how this signal transduction process is regulated. 


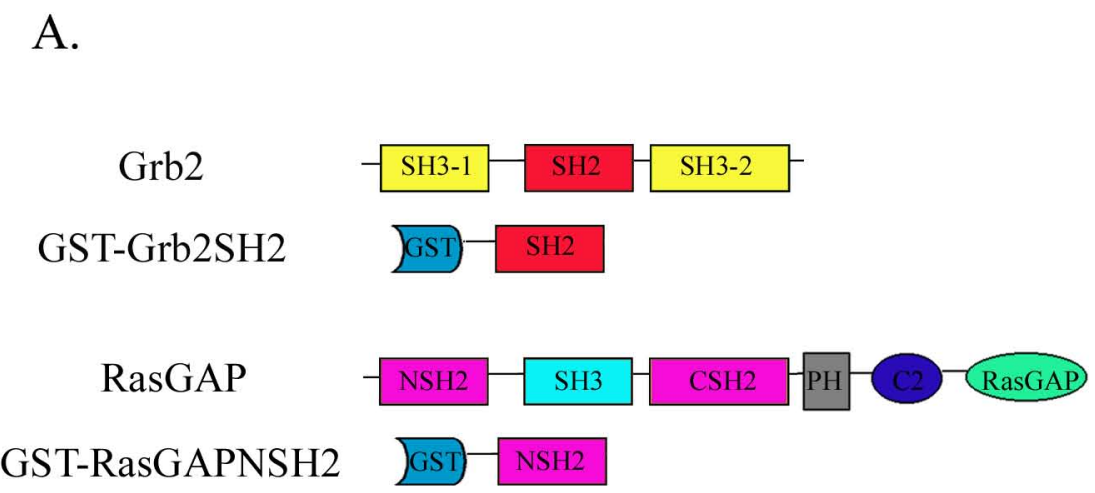

B.

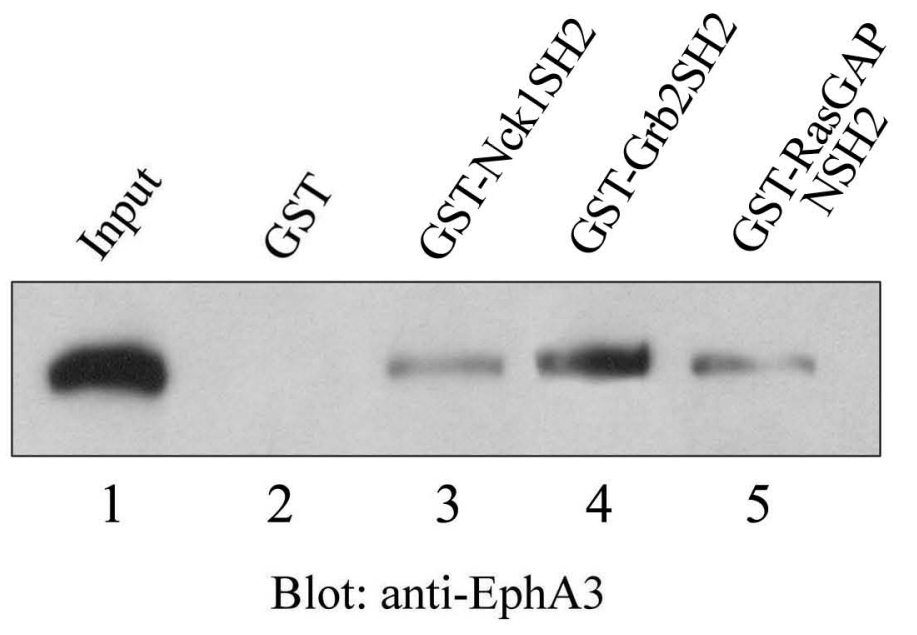


Figure 5-1. Interaction of EphA3 with Grb2 SH2 domain and RasGAP N-terminal SH2 domain.

(A) Domain structure of wild type Grb2, RasGAP, and the GST fusion proteins used in the pulldown assays. (B) Western blot analysis of the pulldown experiment demonstrating that both GST-Grb2SH2 and GST-RasGAPNSH2 precipitate wild type EphA3. 

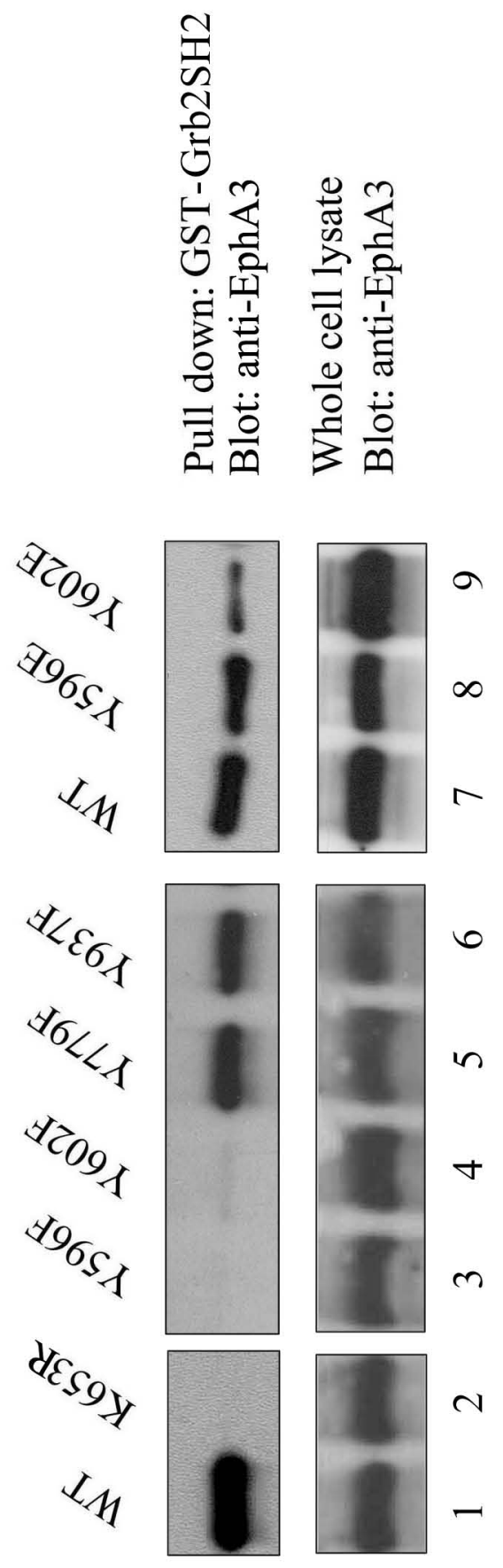
Figure 5-2. Identification of Tyr602 as one binding site of Grb2 protein.

The EphA3 tyrosine mutants along with wild type EphA3 were employed in a pulldown experiment using GST-Grb2SH2. The results were analyzed by Western blot. Tyrosine 602 on EphA3 is one of several binding sites used by Grb2 protein. 

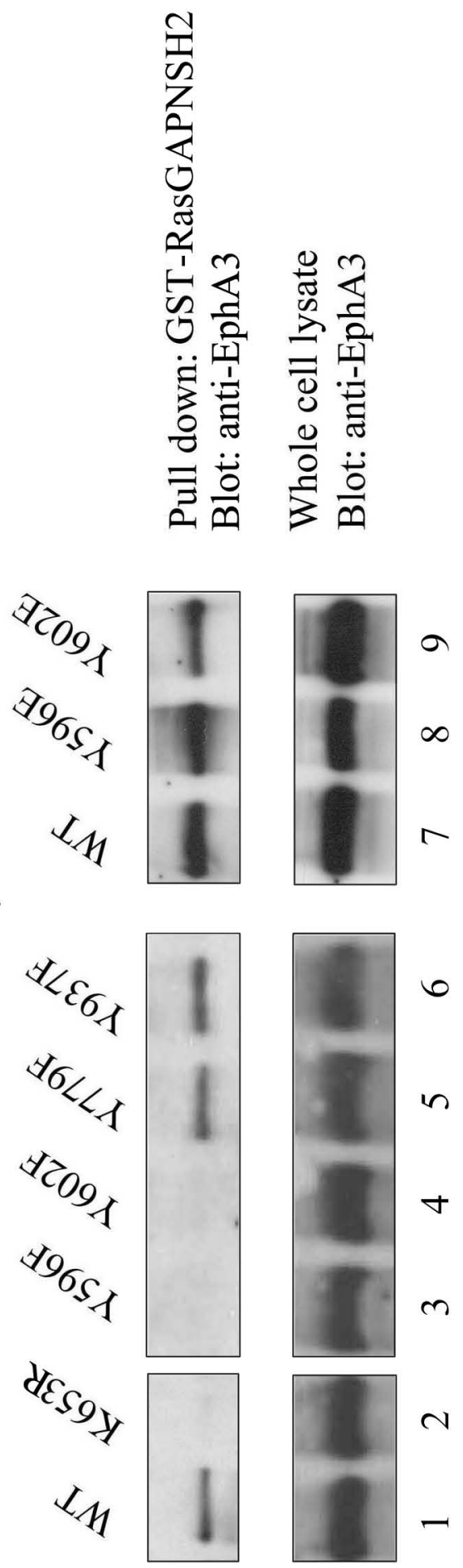
Figure 5-3. Identification of Tyr602 as one of RasGAP binding sites on EphA3.

The results of pulldown assay with GST-RasGAPNSH2 were analyzed by Western blot. RasGAP binds to EphA3 through tyrosine 602 and other unknown sites. 
A. Adaptor proteins

Crk

Grb2

Grb10

Nck1

SHC

SLAP
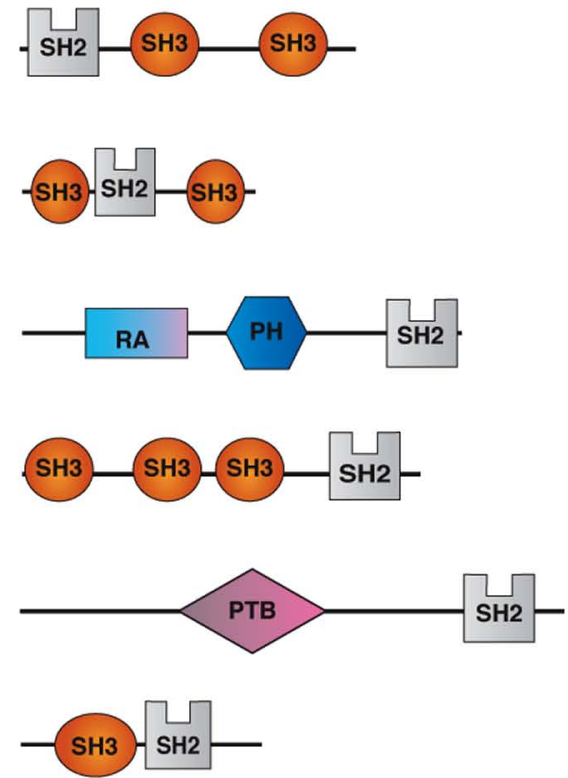

B. Src kinase family

Abl/Arg

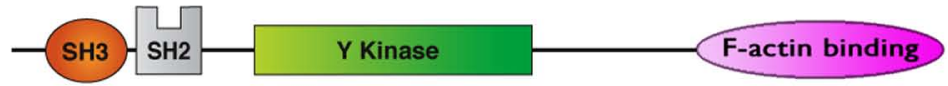

Fyn

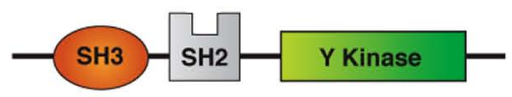

Src

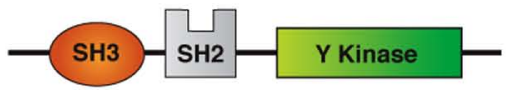

Yes

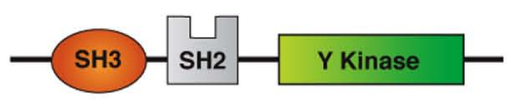

\section{SH2 containing enzymes} p85 PI3Kinase
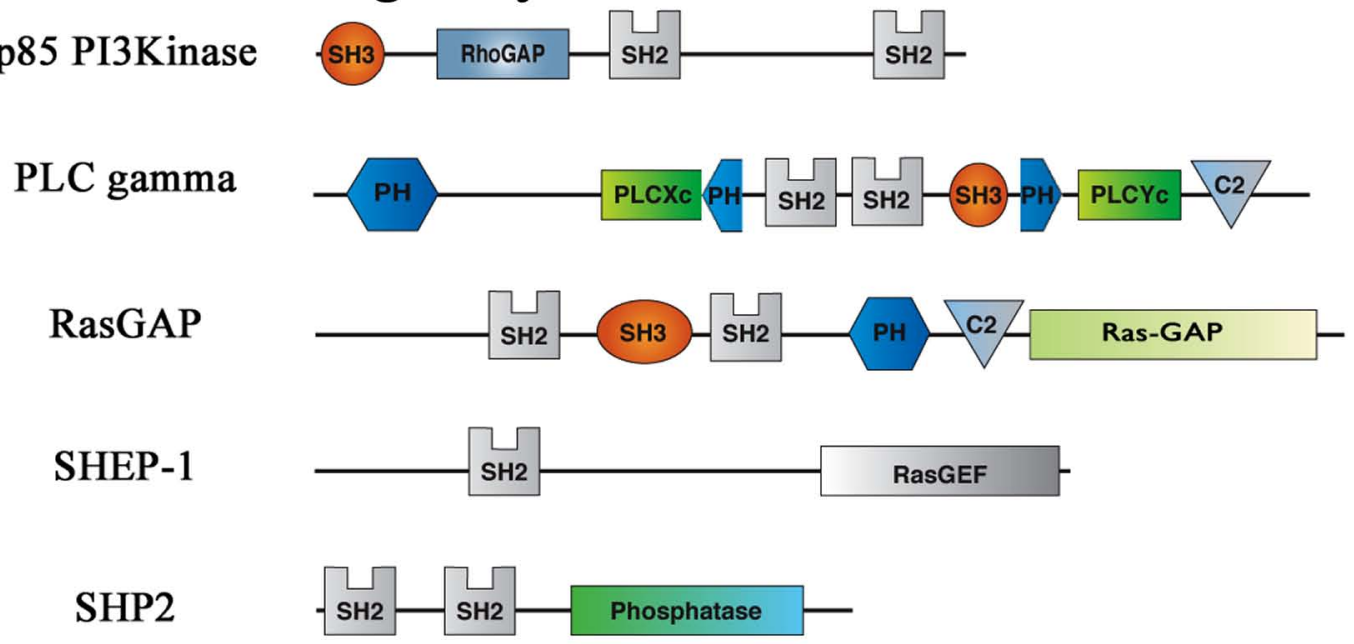
Figure 5-4. Domain structure of all SH2 domain containing proteins that bind to Eph receptors.

(A) Adaptor proteins. (B) Src family kinases (SFKs). (C) Enzyme that associate with Eph receptors. Abbreviations: C2, protein kinase $\mathrm{C}$ conserved region 2; F-actin binding, Factin binding domain; PH, Pleckstrin homology domain; Phosphatase, phosphotyrosine phosphatase domain; PLCXc, phospholipase C catalytic domain; PTB, phosphotyrosine binding domain, RA, Ras-associated-like domain; Ras-GAP, Ras-GAP domain; RasGEF, Ras-GEF domain; RhoGAP, RhoGAP domain; SH2, Src homology-2 domain; SH3, Src homology-3 domain; Y Kinase, tyrosine kinase domain. 


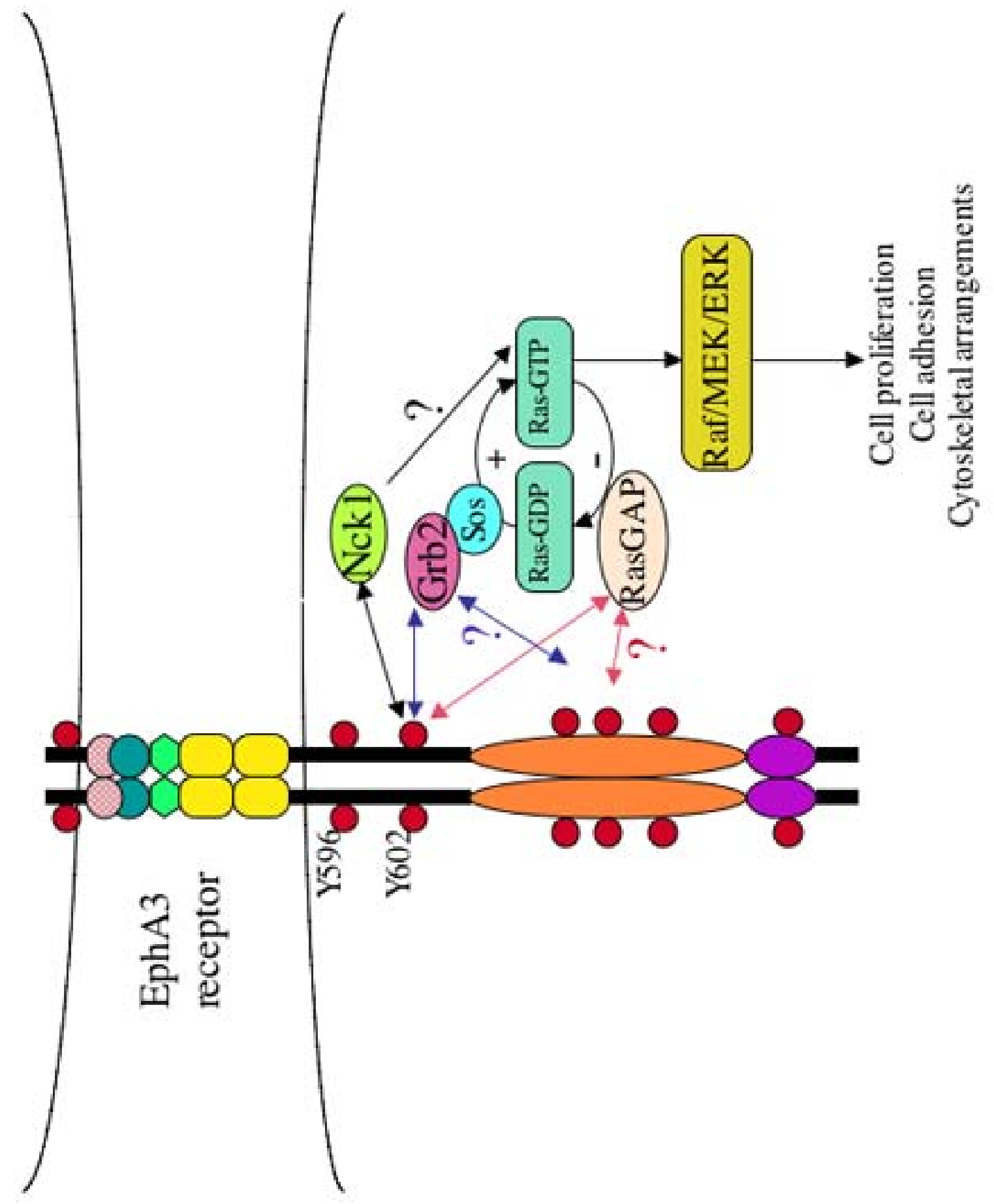


Figure 5-5. A schematic representation of the interactions of $\operatorname{EphA3}$ receptor with Grb2 and RasGAP.

Both Grb2 and RasGAP interact through Y602 as well as other phosphotyrosines at unknown sites. Grb2 and RasGAP regulate MAPK activity oppositely through regulating Ras GTPase activity. Nck1 binds solely through Y602 of EphA3, but its role in Ras/Raf/MAPK pathway is still unclear. 


\section{Chapter VI. Materials and Methods}

\section{Reagents and antibodies}

Ephrin-A5-Fc fusion protein was purchased from R\&D systems (Minneapolis, MN). The ligand was clustered through antibody cross-linking using anti-human Fc (Jackson ImmunoResearch, West Grove, PA) at an antibody to ephrin ratio of 1 to 5 in weight (1:15 in molar ratio). The mixture was incubated at $4{ }^{\circ} \mathrm{C}$ for 8 hours and used to stimulate cells at $1-2 \mu \mathrm{g} / \mathrm{ml}$ concentration. Mouse anti-Nck1 monoclonal antibody was purchased from BD Biosciences (San Jose, CA). The rabbit anti-EphA3 polyclonal antibody and mouse anti-HA monoclonal antibody were purchased from Santa Cruz (Santa Cruz, CA). The phosphotyrosine antibody used in analyzing the phosphorylation of the EphA3 receptors was purchased from Cell Signaling Technology (Danvers, MA). For Western blot analyses, these antibodies were used at 1:1000 and for immunocytochemical staining, at 1:200. Secondary antibodies used in Western blotting and immunostaining were acquired from Sigma-Aldrich (St. Louis, MO) and Jackson ImmunoResearch (West Grove, PA) respectively. When re-blotting was required, the nitrocellulose membrane was washed briefly and incubated in Western-blot re-strip buffer from G-Biosciences (St. Louis, MO).

\section{Yeast two-hybrid screen}

The Yeast two-hybrid screen was performed with DupLex-A system from Origene (Rockville, MD) according to the instructions. In brief, the intracellular domain of EphA3 receptor was cloned into pEG202-NLS vector, fused to DNA binding protein LexA to 
generate the bait plasmid pEG202-NLS-EphA3intra. This plasmid was then transformed into yeast strain EGY188 along with a reporter plasmid carrying a LacZ gene and an embryonic mouse brain cDNA library cloned in the target plasmid pJG4-5. The transformed yeast cells were plated and screened for LacZ transcription through X-gal reaction. All positive clones were then extracted and sequenced. The identity of the positive clones was determined using BLAST searches against public databases. The specificity of the binding between the identified cDNA products and the bait protein used was further confirmed through yeast mating test. Briefly, the isolated target plasmid carrying cDNA fragment was transformed into EGY188 and this strain was mated with an EGY40 strain containing both the reporter plasmid and pEG202-NLS-EphA3intra. Then the offspring cells were lysed and incubated with X-gal solution for 2 hours. Negative controls for mating test were created by using an empty bait plasmid (pBait) or an irrelevant control plasmid (pRHFM) in place of pEG202-NLS-EphA3intra in the test.

\section{Cell culture and DNA transfection}

HEK293A cells were cultured in DMEM supplemented with $10 \%$ fetal bovine serum, penicillin and streptomycin (100 U/ml and $100 \mu \mathrm{g} / \mathrm{ml}$ respectively). When cells grew to about $90 \%$ confluence, plasmid DNA was transfected into the cells using Lipofectamine 2000 (Invitrogen, Carlsbad, CA) according to the manufacturer's instructions. About $10 \mu \mathrm{g}$ DNA and $30 \mu 1$ Lipofectamine 2000 were used to transfect one $100 \mathrm{~mm}$ dish. Six hours later, the transfection mixture was removed and replaced with regular culture medium (DMEM supplemented with 10\% fetal bovine serum). Cells were harvested at 48 hours after transfection for assays. In brief, the cells were washed once 
with ice cold PBS and lysed in lysis buffer $(50 \mathrm{mM}$ Tris $\bullet \mathrm{Cl}, \mathrm{pH} 8.0,150 \mathrm{mM} \mathrm{NaCl}, 1 \%$ NP-40, $100 \mu \mathrm{g} / \mathrm{ml}$ PMSF, $10 \mu \mathrm{g} / \mathrm{ml}$ aprotinin and $10 \mu \mathrm{g} / \mathrm{ml}$ leupeptin, $1 \mathrm{mM}$ sodium orthovanadate) for further analysis. The total protein concentration of these lysates was measured using BioRad Dc protein assay (Hercules, CA).

\section{Analysis of the EphA3 receptor phosphorylation}

Cell lysates of transfected HEK293A cells were pre-cleared by incubation with $70 \mu 1$ $50 \%$ Protein A beads (Upstate Technology, Lake Placid, NY) for 1 hour at $4{ }^{\circ} \mathrm{C}$. About $500 \mu \mathrm{g}$ of total protein was incubated with $2.0 \mu \mathrm{g}$ rabbit polyclonal anti-EphA3 antibody (c-19, Santa Cruz Biotechnology, Santa Cruz, CA). After two hours of incubation with agitation at $4{ }^{\circ} \mathrm{C}, 70 \mu \mathrm{l}$ of $50 \%$ Protein A beads were added and the incubation continued for 1 more hour at $4{ }^{\circ} \mathrm{C}$. Afterwards, the supernatant was disposed and the beads were washed extensively with IP wash buffer (PBS, 0.1\% Triton X-100, $100 \mu \mathrm{g} / \mathrm{ml}$ PMSF, 10 $\mu \mathrm{g} / \mathrm{ml}$ Aprotinin, $10 \mu \mathrm{g} / \mathrm{ml}$ Leupeptin, and $1 \mathrm{mM}$ Sodium orthovanadate). At the end of the washes, the beads were mixed with equal volume of $2 \mathrm{X}$ Laemmli buffer and boiled for 5 minutes. The phosphorylation levels of the various EphA3 mutant proteins were analyzed with Western blot using the anti-phosphotyrosine antibody (Cell Signaling, Danvers, MA).

\section{Preparation of Glutathione S-Transferase (GST) fusion protein}

Different GST fusion constructs in pGEX 4T-2 (Li, Meriane et al. 2002) were transformed into competent DH5 $\alpha$ bacteria cells. To produce the GST fusion proteins, a single bacteria colony was used to inoculate $5 \mathrm{ml} 2 x$ YTA medium (16 g/l Tryptone, 10 
$\mathrm{g} / \mathrm{l}$ Yeast extract, $5 \mathrm{~g} / 1 \mathrm{NaCl}$ and $100 \mu \mathrm{g} / \mathrm{ml}$ Ampicillin) and cultured overnight at $30^{\circ} \mathrm{C}$. On the second day, the culture was used to inoculate $500 \mathrm{ml} 2$ xYTA culture medium, which was incubated at $30{ }^{\circ} \mathrm{C}$ until the OD at $600 \mathrm{~nm}$ reached $0.5-2.0$. Then IPTG was added to a final concentration of $1 \mathrm{mM}$ to induce expression of GST fusion proteins. The culture was incubated for another 6 hours at $30^{\circ} \mathrm{C}$. At the end of incubation, the bacteria were collected by centrifugation at $8,000 \mathrm{rpm}$ using a Sorvall centrifuge (Thermo Scientific, Waltham, MA). Bacteria pellet from $100 \mathrm{ml}$ cultures was re-suspended in $5 \mathrm{ml}$ PBS, supplemented with protease inhibitors $(100 \mu \mathrm{g} / \mathrm{ml} \mathrm{PMSF,} 10 \mu \mathrm{g} / \mathrm{ml}$ aprotinin and $10 \mu \mathrm{g} / \mathrm{ml}$ leupeptin) and sonicated on ice in short bursts. Triton X-100 was then added to the sonicated product at a final concentration of $1 \%$ and the cell lysates were incubated at $4{ }^{\circ} \mathrm{C}$ for 30 minutes with agitation. Bacteria cell debris was removed by centrifugation and the supernatant was incubated with $200 \mu 1$ 50\% Glutathione Sepharose beads (GE healthcare Pharmacia, Piscataway, NJ) at $4{ }^{\circ} \mathrm{C}$ for 1 hour. The beads were washed with PBS containing $0.05 \%$ Triton $\mathrm{X}-100$ for 3 times and stored in PBS with protease inhibitors $\left(100 \mu \mathrm{g} / \mathrm{ml}\right.$ PMSF, $10 \mu \mathrm{g} / \mathrm{ml}$ aprotinin and $10 \mu \mathrm{g} / \mathrm{ml}$ leupeptin) at $4{ }^{\circ} \mathrm{C}$ until use.

\section{GST fusion protein pulldown}

Transfected 293A cells were stimulated with cross-linked ephrin-A5-Fc (1 $\mu \mathrm{g} / \mathrm{ml})$ for 30 minutes at $37{ }^{\circ} \mathrm{C}$. The cells were washed once with ice cold PBS and lysed in GST pulldown assay lysis buffer (10 mM Hepes, pH 7.4, 1\% Triton X-100, 1 mM PMSF, 10 $\mu \mathrm{g} / \mathrm{ml}$ aprotinin and $10 \mu \mathrm{g} / \mathrm{ml}$ leupeptin, $1 \mathrm{mM}$ sodium orthovanadate). The lysate was collected with a cell scraper and centrifuged at 13,000 rpm for 10 minutes in an 
Eppendorf microcentrifuge. After centrifugation to remove cell debris, the supernatant was pre-cleared with $50 \mu \mathrm{l}$ of $50 \%$ Glutathione Sepharose beads for 30 minutes at $4{ }^{\circ} \mathrm{C}$. Later, Glutathione Sepharose beads conjugated with the desired GST fusion proteins were added to the supernatant and the mixture was incubated at $4{ }^{\circ} \mathrm{C}$ for 2 hours. At the end of incubation, the beads were collected by centrifugation and washed 3 times with PBS supplemented with $0.05 \%$ Triton X-100, each for 10 minutes. The beads were then boiled in 2X Laemmli buffer for 5 minutes before analysis by Western blot technique.

\section{Co-immunoprecipitation assay}

Transfected 293A cells or dissociated neuron cultures were stimulated with or without cross-linked ephrin-A5-Fc for 30 minutes at $37^{\circ} \mathrm{C}$. The cells were washed gently with ice cold PBS once and lysed in cell lysis buffer. The cell lysate then was cleared by centrifugation and the protein concentration of the supernatant determined. A small fraction of the lysate was used later for analysis of protein expression levels. For immunoprecipitation, $2 \mathrm{mg}$ of each lysate were pre-cleared with protein A beads (Upstate USA, Inc., Charlottesville, VA) for 1 hour at $4{ }^{\circ} \mathrm{C}$. Then the desired antibody or control IgG was added to the supernatant. The mixture was incubated on a rocker at $4{ }^{\circ} \mathrm{C}$ for 4 hours before $40 \mu 150 \%$ protein A beads were added. After continued incubation of 1 more hour, the beads were collected and washed with PBS with $0.05 \%$ Triton X-100 for 3 times, each 10 minutes. The precipitated immunocomplexes were boiled in $2 \mathrm{X}$ Laemmli buffer for 5 minutes and the samples were analyzed with SDS-PAGE coupled with Western blot technique using different antibodies. 


\section{Immunostaining}

Cells were stimulated with cross-linked ephrin-A5 for 30 minutes and fixed with freshly prepared $4 \%$ paraformaldehyde solution for 30 minutes at room temperature. The fixed cells were washed two times with PBS, each 10 minutes. The cells were then blocked with $5 \%$ BSA in PBS plus $0.05 \%$ Triton X-100 overnight at $4{ }^{\circ} \mathrm{C}$. On the second day, blocking buffer was replaced with primary antibodies in $0.5 \mathrm{X}$ blocking buffer $(2.5 \%$ BSA and $0.025 \%$ Triton X-100 in PBS). After 4 hours of incubation at room temperature, the cells were washed again 2 times with PBS and the secondary antibody in $0.5 \mathrm{X}$ blocking buffer was added and incubated for 2 hours. Two more washes follow the antibody incubation and DAPI solution $(1 \mu \mathrm{g} / \mathrm{ml})$ was used to stain the cells for 30 minutes at room temperature to visualize the nuclei. After two additional PBS washes, the culture dishes were examined under a fluorescence microscope.

\section{Cellular process retraction assay}

HEK293A cells were plated sparsely on culture dishes to allow examination of individual cells and their processes. These cells were transfected with desired plasmids and 48 hours after transfection, the cells were incubated in DMEM medium with or without cross-linked ephrin-A5 for 1 hour at $37^{\circ} \mathrm{C}$. Pictures of transfected cells $(\mathrm{N}>30)$ as indicated by EGFP expression, were taken before and after the treatment (L1, L2, respectively). The length of processes was measured from where they exit the cell body to the end of the process using ImagePro software. Using the formula $\mathrm{E}$ (extension) $=$ (L2 - L1)/ L1, we calculated the process length change index E as a percentage of initial total process length. A positive $\mathrm{E}$ value indicates process extension and a negative number 
indicates process retraction. E value of transfected cells was compared to EGFP expressing cells (100\%) and then plotted in the bar graph.

\section{Cell migration assay}

To prepare for this assay, transwell inserts $(24.5 \mathrm{~mm}$ diameter, $8 \mu \mathrm{m}$ pore diameter) purchased from Corning Labware (Corning, NY) were coated on the underside with fibronectin $(20 \mu \mathrm{g} / \mathrm{ml})$ to facilitate attachment of migrated cells. HEK293A cells were transfected with desired genes using Lipofectamine 2000 reagent according to manufacturer's instructions (Invitrogen, Carlsbad, CA). Twenty-four hours after transfection, the cells were trypsinized and washed gently with PBS. A small portion of the cells was plated into 6 well dishes for determination of efficiency for each transfection. An equal portion of the cell suspension was used in the migration assay. They were plated on the prepared transwell inserts in DMEM without serum and the lower compartment in the setup was filled with complete culture medium (DMEM supplemented with $10 \%$ FBS) with or without $2 \mu \mathrm{g} / \mathrm{ml}$ cross-linked ephrin-A5. These transwell dishes were then incubated overnight at $37{ }^{\circ} \mathrm{C}$ with $5 \% \mathrm{CO}_{2}$. On the second day, cells on inserts were fixed and all the cells that have not migrated were cleaned off using Q-tips. DAPI staining was carried out to visualize the cell nuclei before cell counting was done on a microscope. Five to ten random fields were chosen and the number of all cells that migrated through the membrane (T2) and the number of the transfected cells that migrated (T1) were both quantified. In addition, the transfection efficiency was individually determined and expressed as a percentage $(\mathrm{R}=$ transfected cells/all cells). The migration ability (M) of cells expressing different genes was 
calculated using the following formula: $\mathrm{M}=\mathrm{T} 1 /(\mathrm{T} 2 \times \mathrm{R})$. When the data were used in the graph, $\mathrm{M}$ was compared with EGFP transfected controls and converted as a percentage of the $M$ value of EGFP control, which is considered to be 1 .

\section{Dissection of rat embryos and neuron culture}

Embryonic day 18 Sprague-Dawley rats were sacrificed and the brains were taken out. After midbrain was trimmed, the two hemispheres were separated and turned over. The cortices and hippocampi were dissected out and collected. To make dissociated neuron culture, the collected neural tissues were trypsinized at $37^{\circ} \mathrm{C}$ for 10 minutes and dissociated further by pipetteing repeatedly with glass pipettes with small opening. At the end, dissociated neurons were counted and plated in neurobasal medium (1 X B27 supplement, $0.5 \mathrm{mM}$ glutamine, $25 \mu \mathrm{M}$ glutamate, $100 \mathrm{U} / \mathrm{ml}$ penicillin and $100 \mu \mathrm{g} / \mathrm{ml}$ streptomycin) at desirable density on culture dishes that were coated with Poly-D-lysine (100 $\mu \mathrm{g} / \mathrm{ml})$. For staining experiment, a low-density culture was used to allow easy observation of individual neurons. For Western blotting and immunoprecipitation, a large quantity of cells was plated at a very high density to ensure enough proteins would be available for assays. To make explant culture, the hippocampi were cut into small pieces until a uniform size of $1 \mathrm{~mm}$ in diameter was achieved. These explants were gently pipetted and plated in neurobasal medium on Nunc Lab-Tek 4-well glass chamber slides that were coated with Poly-D-lysine $(500 \mu \mathrm{g} / \mathrm{ml})$ and Laminin $(20 \mu \mathrm{g} / \mathrm{ml})$.

\section{Adenovirus construction}

In a nutshell, the various Nck1 genes including Nck1WT, Nck1R308K and Nck1W38/143/229K were cloned into pAdTrack-CMV vector. These plasmids carrying 
Nck1 mutants were individually linerized through PmeI digestion and cleaned through agarose gel extraction. A mixture of the linerized pAdTrack-CMV-Nck1 clones and pAdEasy vector was used to transform BL5781 cells in which recombination of the two plasmids took place. Individual colonies were picked out and screened for the right recombinant products, Recombinant adenoviral vectors, pAdEasy-CMV, pAdEasy-CMV-Nck1WT, pAdEasyCMV-Nck1R308K and pAdEasy-CMV-Nck1W38/143/229K were each digested with PacI enzyme and the digested plasmids were used to transfect adenovirus packaging cell line HEK293A cells. Seven days later, the transfected cells were collected and processed through "freeze-thaw-vortex" procedure to release all adenoviruses from the cells. In brief, harvested cell pellets were resuspended in sterile PBS and frozen in a dry ice/ethanol bath for 5 minutes. The frozen cells were then thawed at $37^{\circ} \mathrm{C}$ and vortexed vigorously until a homogenous suspension was obtained. This "freeze-thaw-vortex" procedure was repeated at least three times before being spun at $5000 \mathrm{rpm}$ for 5 minutes. After being ridden of the cell debris, the supernatants were used to infect 293 A cells. The infected cells were harvested and processed to releases the amplified adenoviruses. This amplification process was repeated until a high titer of virus stock was obtained. The amplified virus stocks were stored at -80 ${ }^{\circ} \mathrm{C}$.

\section{Growth cone collapse}

Hippocampal explant culture was infected with adenoviruses on the same day they were plated. After overnight infection, fresh culture medium was added to the culture. Four days later, cross-linked ephrin-A5 was used to treat these explants for half an hour before a freshly mixed fixative (4\% paraformaldehyde, $0.25 \%$ glutaraldehyde, $0.1 \mathrm{M}$ cacodylate buffer $\mathrm{pH} 7.4$ 
and $0.1 \mathrm{M}$ sucrose) was used to fix the cultures. The explant cultures were washed three times with PBS before being incubated in $0.3 \%$ Triton-X-100 in PBS for 20 minutes. Texas Red X-Phalloidin was used to stain the cells for 20 minutes at room temperature. The cultures were washed again with PBS until all the residual stain was cleaned. To prepare the slides for inspection on a microscope, the top chambers were removed from the chamber slides and a small amount of $50 \%$ glycerol in PBS was added on top of the slide. Glass coverslips were then mounted on these slides and the edges of the coverslips were sealed with nail polish. These slides were wrapped in foil and kept at $4{ }^{\circ} \mathrm{C}$ until use. 


\section{Chapter VII. Conclusions}

\section{Novel binding proteins are implicated in EphA receptor signaling}

The mechanisms of Eph/ephrin signaling remain an important topic of research as increasing amounts of data suggest that the complexity of Eph/ephrin signal transduction surpasses our initial expectations. Though many binding proteins have been identified, our understanding of the topic remains incomplete.

Through a large-scale yeast two-hybrid screen, we identify fifteen novel proteins that interact with the cytoplasmic domain of EphA receptors. These proteins are categorized into four groups: membrane trafficking related genes, cytoskeleton regulation related genes, tumorigenesis related genes, and cytoplasmic enzymes. These proteins likely participate in the regulation of various functions Ephs regulate. Further studies on these novel proteins will increase our understanding of the mechanisms involved in modulating a variety of cellular events such as endocytosis, cytoskeletal rearrangements and cell proliferation.

\section{Nck1, Grb2 and RasGAP all bind to tyrosine 602 through their SH2 domains}

The present study identifies adaptor protein Nck1 as a major binding protein for both EphA3 and EphA5. Nck1 has been found to bind to EphB1 and EphB2 through direct or indirect interaction (Stein et al. 1998a); however, its involvement in EphA receptors has never been established.

The current study also shows that Nck1 only interacts with tyrosine residue 602 of EphA3 receptor. Tyr602 is located within the juxtamembrane domain of EphA3 receptor 
and is highly conserved among all Eph receptors. The interaction of Nck1 with the juxtamembrane domain of Eph receptors is common to many $\mathrm{SH} 2$ domain proteins. Since a diversity of biological events are mediated by SH2 domain proteins, the competition between Nck1 and other SH2 domain proteins for binding with the juxtamembrane domain is a probable explanation for the drastically different phenotypes induced by activated EphA receptors.

Grb2 and RasGAP are two SH2 domain proteins involved in the regulation of MAPK activity. Although this study has yet to examine all sites to which Grb2 and RasGAP bind, we show that these two signaling proteins share at least one binding site.. The preferential association with either Grb2 or RasGAP is likely to result in up- or downregulation of Ras/ERK/MAPK pathway and present an underlying mechanisms for the switch between attractive and repulsive phenotypes (Tong et al. 2003).

It remains to be determined which roles Nck1 plays in this regulatory process. Though Nck1 is known to bind to Sos and induce the activation of Ras activity, Nck1 has also been identified in a RasGAP/Nck1/EphB2 protein complex. Taken together, these data suggest that Nck1 is capable of exerting opposite effects in a context dependent fashion (Tanaka et al. 1995a).

\section{Nck1 is an integral part of EphA3 mediated signaling}

Nck1 co-localizes with EphA3 after ephrin stimulation in both HEK 293A cells and primary neuron cultures, indicating the endogenous association of the two proteins. Nck1 binds to activated EphA3 and triggers the activation of downstream signaling proteins such as PAK, NIK, Nap-1, and WASp/WAVE proteins (Fig. 7-1). The activation of these 
signaling pathways in turn leads to changes such as cytoskeletal rearrangement and cell adhesion.

When dominant negative Nck1 genes are introduced into 293A cells, the function of endogenous Nck1 is compromised. Furthermore, it is found that the disruption of either Nck1/EphA3 binding or Nck1/effector binding is able to elicit a partial reversal of Eph mediated phenotypes such as inhibition on cell migration and process retraction after ephrin treatment.

Although the significance of Nck1 in EphA3 mediated growth cone collapse wasn't confirmed in this study, it remains probable that Nck1 functions in other aspects of axonal behavior such as axonal branching, neurite retraction, turning and synaptic formation. More research needs to be conducted to elucidate the signaling mechanism of Nck1 in EphA3 expressing neurons. 


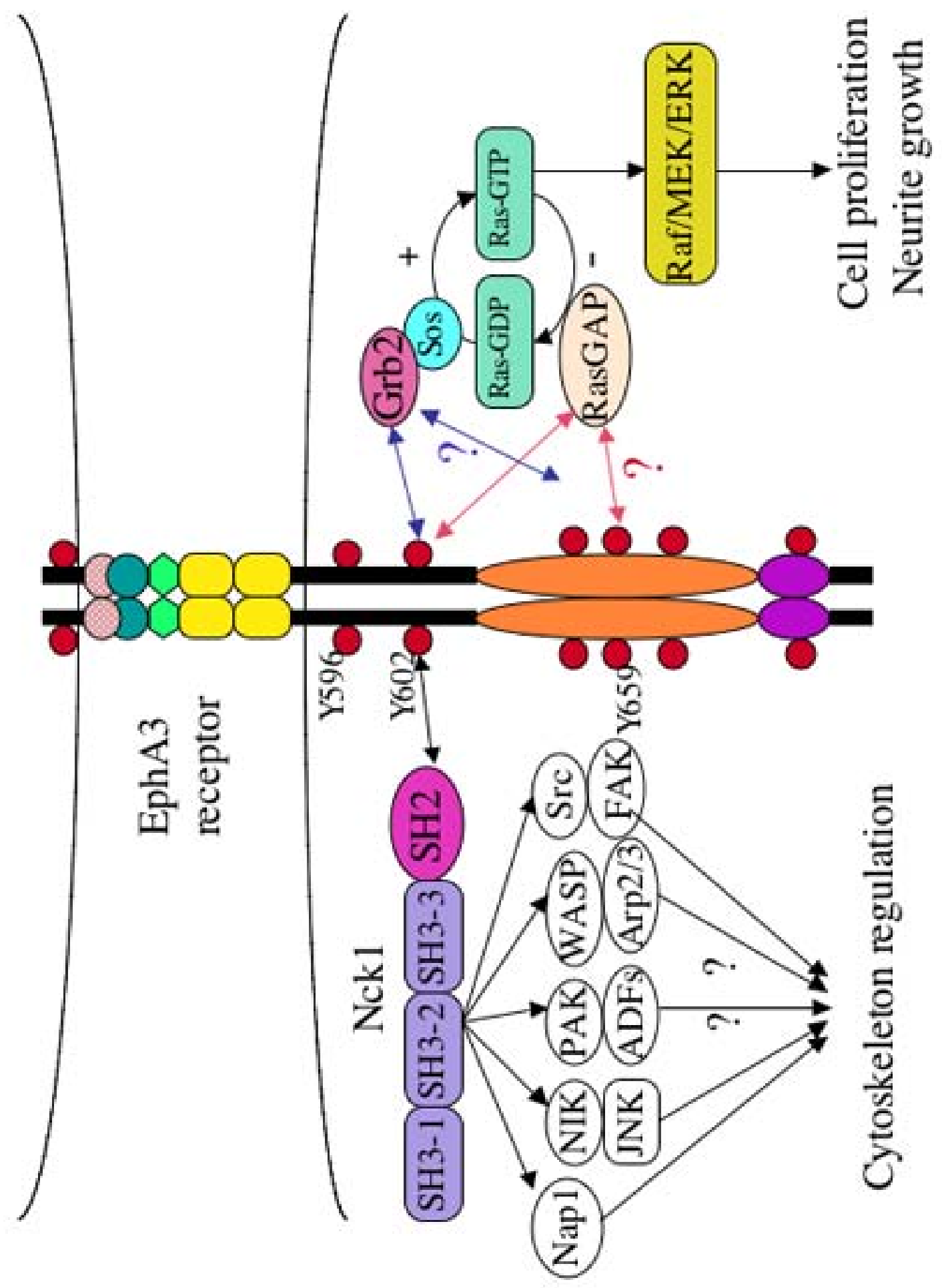


Figure 7-1. A summary of EphA3 signal transduction pathway involving Nck1, Grb2 and RasGAP.

Signaling pathways downstream of EphA3 that are regulated by Nck1, Grb2 and

RasGAP are delineated in the graph. Through multiple pathways, Nck1 binds to Tyr602 and regulates cytoskeletal activities. Grb2 and RasGAP, on the other hand, compete for binding through both Y602 and unknown binding sites, thereby modulating Ras/MEK/ERK pathway. 


\section{References}

Aasheim H.C., Patzke S., Hjorthaug H.S. \& Finne E.F. (2005) Characterization of a novel Eph receptor tyrosine kinase, EphA10, expressed in testis. Biochim Biophys Acta 1723:1-7.

Adams R.H. \& Klein R. (2000) Eph receptors and ephrin ligands. essential mediators of vascular development. Trends Cardiovasc Med 10:183-188.

Andres A.C., Reid H.H., Zurcher G., Blaschke R.J., Albrecht D. \& Ziemiecki A. (1994)

Expression of two novel eph-related receptor protein tyrosine kinases in mammary gland development and carcinogenesis. Oncogene 9:1461-1467.

Anton I.M., Lu W., Mayer B.J., Ramesh N. \& Geha R.S. (1998) The Wiskott-Aldrich syndrome protein-interacting protein (WIP) binds to the adaptor protein Nck. J Biol Chem 273:20992-20995.

Aoki M., Yamashita T. \& Tohyama M. (2004) EphA receptors direct the differentiation of mammalian neural precursor cells through a mitogen-activated protein kinasedependent pathway. J Biol Chem 279:32643-32650.

Avruch J., Khokhlatchev A., Kyriakis J.M., Luo Z., Tzivion G., Vavvas D. \& Zhang X.F. (2001) Ras activation of the Raf kinase: tyrosine kinase recruitment of the MAP kinase cascade. Recent Prog Horm Res 56:127-155.

Bagrodia S., Taylor S.J., Creasy C.L., Chernoff J. \& Cerione R.A. (1995) Identification of a mouse p21Cdc42/Rac activated kinase. J Biol Chem 270:22731-22737.

Barbieri M.A., Ramkumar T.P., Fernadez-Pol S., Chen P.I. \& Stahl P.D. (2004) Receptor tyrosine kinase signaling and trafficking--paradigms revisited. Curr Top Microbiol Immunol 286:1-20.

Barr A.J. \& Knapp S. (2006) MAPK-specific tyrosine phosphatases: new targets for drug discovery? Trends Pharmacol Sci 27:525-530.

Batlle E., Bacani J., Begthel H., Jonkheer S., Gregorieff A., van de Born M., Malats N., Sancho E., Boon E., Pawson T., Gallinger S., Pals S. \& Clevers H. (2005) EphB receptor activity suppresses colorectal cancer progression. Nature 435:1126-1130.

Becker E., Huynh-Do U., Holland S., Pawson T., Daniel T.O. \& Skolnik E.Y. (2000) Nck-interacting Ste20 kinase couples Eph receptors to c-Jun N-terminal kinase and integrin activation. Mol Cell Biol 20:1537-1545.

Beckmann M.P., Cerretti D.P., Baum P., Vanden Bos T., James L., Farrah T., Kozlosky C., Hollingsworth T., Shilling H., Maraskovsky E. \& et al. (1994) Molecular characterization of a family of ligands for eph-related tyrosine kinase receptors. Embo J 13:3757-3762.

Beggs H.E., Schahin-Reed D., Zang K., Goebbels S., Nave K.A., Gorski J., Jones K.R., Sretavan D. \& Reichardt L.F. (2003) FAK deficiency in cells contributing to the basal lamina results in cortical abnormalities resembling congenital muscular dystrophies. Neuron 40:501-514.

Beites C.L., Xie H., Bowser R. \& Trimble W.S. (1999) The septin CDCrel-1 binds syntaxin and inhibits exocytosis. Nat Neurosci 2:434-439.

Birge R.B., Knudsen B.S., Besser D. \& Hanafusa H. (1996) SH2 and SH3-containing adaptor proteins: redundant or independent mediators of intracellular signal transduction. Genes Cells 1:595-613. 
Bladt F., Aippersbach E., Gelkop S., Strasser G.A., Nash P., Tafuri A., Gertler F.B. \& Pawson T. (2003) The murine Nck SH2/SH3 adaptors are important for the development of mesoderm-derived embryonic structures and for regulating the cellular actin network. Mol Cell Biol 23:4586-4597.

Blits-Huizinga C.T., Nelersa C.M., Malhotra A. \& Liebl D.J. (2004) Ephrins and their receptors: binding versus biology. IUBMB Life 56:257-265.

Bokoch G.M. (2003) Biology of the p21-activated kinases. Annual Review of Biochemistry 72:743-781.

Bokoch G.M., Wang Y., Bohl B.P., Sells M.A., Quilliam L.A. \& Knaus U.G. (1996) Interaction of the Nck adapter protein with p21-activated kinase (PAK1). J Biol Chem 271:25746-25749.

Boyd A.W. \& Lackmann M. (2001) Signals from Eph and ephrin proteins: a developmental tool kit. Sci STKE 2001:RE20.

Brambilla R. \& Klein R. (1995) Telling axons where to grow: a role for Eph receptor tyrosine kinases in guidance. Mol Cell Neurosci 6:487-495.

Brantley D.M., Cheng N., Thompson E.J., Lin Q., Brekken R.A., Thorpe P.E., Muraoka R.S., Cerretti D.P., Pozzi A., Jackson D., Lin C. \& Chen J. (2002) Soluble Eph A receptors inhibit tumor angiogenesis and progression in vivo. Oncogene 21:70117026.

Brantley-Sieders D., Schmidt S., Parker M. \& Chen J. (2004) Eph receptor tyrosine kinases in tumor and tumor microenvironment. Curr Pharm Des 10:3431-3442.

Brent R. \& Ptashne M. (1985) A eukaryotic transcriptional activator bearing the DNA specificity of a prokaryotic repressor. Cell 43:729-736.

Brown A., Yates P.A., Burrola P., Ortuno D., Vaidya A., Jessell T.M., Pfaff S.L., O'Leary D.D. \& Lemke G. (2000) Topographic mapping from the retina to the midbrain is controlled by relative but not absolute levels of EphA receptor signaling. Cell 102:77-88.

Bruce V., Olivieri G., Eickelberg O. \& Miescher G.C. (1999) Functional activation of EphA5 receptor does not promote cell proliferation in the aberrant EphA5 expressing human glioblastoma U-118 MG cell line. Brain Res 821:169-176.

Bruckner K. \& Klein R. (1998) Signaling by Eph receptors and their ephrin ligands. Curr Opin Neurobiol 8:375-382.

Bruckner K., Pablo Labrador J., Scheiffele P., Herb A., Seeburg P.H. \& Klein R. (1999) EphrinB ligands recruit GRIP family PDZ adaptor proteins into raft membrane microdomains. Neuron 22:511-524.

Bruckner K., Pasquale E.B. \& Klein R. (1997) Tyrosine phosphorylation of transmembrane ligands for Eph receptors. Science 275:1640-1643.

Bu X., Avraham H.K., Li X., Lim B., Jiang S., Fu Y., Pestell R.G. \& Avraham S. (2005) Mayven induces c-Jun expression and cyclin D1 activation in breast cancer cells. Oncogene 24:2398-2409.

Buchert M., Schneider S., Meskenaite V., Adams M.T., Canaani E., Baechi T., Moelling K. \& Hovens C.M. (1999) The junction-associated protein AF-6 interacts and clusters with specific Eph receptor tyrosine kinases at specialized sites of cell-cell contact in the brain. J Cell Biol 144:361-371.

Buday L., Egan S.E., Rodriguez Viciana P., Cantrell D.A. \& Downward J. (1994) A complex of Grb2 adaptor protein, Sos exchange factor, and a 36-kDa membrane- 
bound tyrosine phosphoprotein is implicated in ras activation in T cells. J Biol Chem 269:9019-9023.

Buday L., Wunderlich L. \& Tamas P. (2002a) The Nck family of adapter proteins: regulators of actin cytoskeleton. Cell Signal 14:723-731.

Buday L., Wunderlich L. \& Tamas P. (2002b) The Nck family of adapter proteins. Regulators of actin cytoskeleton. Cell Signal 14:723-731.

Burridge K., Fath K., Kelly T., Nuckolls G. \& Turner C. (1988) Focal adhesions: transmembrane junctions between the extracellular matrix and the cytoskeleton. Annu Rev Cell Biol 4:487-525.

Byrne J.L., Paterson H.F. \& Marshall C.J. (1996) p21Ras activation by the guanine nucleotide exchange factor Sos, requires the Sos/Grb2 interaction and a second ligand-dependent signal involving the Sos N-terminus. Oncogene 13:2055-2065.

Caras I.W. (1997) A link between axon guidance and axon fasciculation suggested by studies of the tyrosine kinase receptor EphA5/REK7 and its ligand ephrin-A5/AL1. Cell Tissue Res 290:261-264.

Carlomagno F. \& Santoro M. (2005) Receptor tyrosine kinases as targets for anticancer therapeutics. Curr Med Chem 12:1773-1781.

Carpenter C.L. \& Cantley L.C. (1996) Phosphoinositide kinases. Curr Opin Cell Biol 8:153-158.

Carter N., Nakamoto T., Hirai H. \& Hunter T. (2002) EphrinA1-induced cytoskeletal reorganization requires FAK and p130(cas). Nat Cell Biol 4:565-573.

Ceresa B.P. (2006) Regulation of EGFR endocytic trafficking by rab proteins. Histol Histopathol 21:987-993.

Chattopadhyay A., Vecchi M., Ji Q., Mernaugh R. \& Carpenter G. (1999) The role of individual SH2 domains in mediating association of phospholipase C-gamma1 with the activated EGF receptor. J Biol Chem 274:26091-26097.

Chen M., She H., Kim A., Woodley D.T. \& Li W. (2000) Nckbeta adapter regulates actin polymerization in NIH $3 \mathrm{~T} 3$ fibroblasts in response to platelet-derived growth factor bb. Mol Cell Biol 20:7867-7880.

Chen Z.Y., Sun C., Reuhl K., Bergemann A., Henkemeyer M. \& Zhou R. (2004) Abnormal hippocampal axon bundling in EphB receptor mutant mice. J Neurosci 24:2366-2374.

Cheng H.J., Nakamoto M., Bergemann A.D. \& Flanagan J.G. (1995) Complementary gradients in expression and binding of ELF-1 and Mek4 in development of the topographic retinotectal projection map. Cell 82:371-381.

Cheung A.Y., Chen C.Y., Glaven R.H., de Graaf B.H., Vidali L., Hepler P.K. \& Wu H.M. (2002) Rab2 GTPase regulates vesicle trafficking between the endoplasmic reticulum and the Golgi bodies and is important to pollen tube growth. Plant Cell 14:945-962.

Chien C.T., Bartel P.L., Sternglanz R. \& Fields S. (1991) The two-hybrid system: a method to identify and clone genes for proteins that interact with a protein of interest. Proc Natl Acad Sci U S A 88:9578-9582.

Chin-Sang I.D., George S.E., Ding M., Moseley S.L., Lynch A.S. \& Chisholm A.D. (1999) The ephrin VAB-2/EFN-1 functions in neuronal signaling to regulate epidermal morphogenesis in C. elegans. Cell 99:781-790. 
Choi S., Jeong J., Kim T. \& Park S. (1999) Characterization of ephrin-A1 and ephrin-A4 as ligands for the EphA8 receptor protein tyrosine kinase. Mol Cells 9:440-445.

Chong L.D., Park E.K., Latimer E., Friesel R. \& Daar I.O. (2000) Fibroblast growth factor receptor-mediated rescue of $\mathrm{x}$-ephrin B1-induced cell dissociation in Xenopus embryos. Mol Cell Biol 20:724-734.

Ciossek T., Monschau B., Kremoser C., Loschinger J., Lang S., Muller B.K., Bonhoeffer F. \& Drescher U. (1998) Eph receptor-ligand interactions are necessary for guidance of retinal ganglion cell axons in vitro. Eur J Neurosci 10:1574-1580.

Clark E.A. \& Brugge J.S. (1995) Integrins and signal transduction pathways: the road taken. Science 268:233-239.

Conover J.C., Doetsch F., Garcia-Verdugo J.M., Gale N.W., Yancopoulos G.D. \& Alvarez-Buylla A. (2000) Disruption of Eph/ephrin signaling affects migration and proliferation in the adult subventricular zone. Nat Neurosci 3:1091-1097.

Cowan C.A. \& Henkemeyer M. (2001) The SH2/SH3 adaptor Grb4 transduces B-ephrin reverse signals. Nature 413:174-179.

Cowan C.A., Yokoyama N., Saxena A., Chumley M.J., Silvany R.E., Baker L.A., Srivastava D. \& Henkemeyer M. (2004) Ephrin-B2 reverse signaling is required for axon pathfinding and cardiac valve formation but not early vascular development. Dev Biol 271:263-271.

Cowan C.W., Shao Y.R., Sahin M., Shamah S.M., Lin M.Z., Greer P.L., Gao S., Griffith E.C., Brugge J.S. \& Greenberg M.E. (2005) Vav family GEFs link activated Ephs to endocytosis and axon guidance. Neuron 46:205-217.

Dai Z. \& Pendergast A.M. (1995) Abi-2, a novel SH3-containing protein interacts with the c-Abl tyrosine kinase and modulates c-Abl transforming activity. Genes Dev 9:2569-2582.

Dail M., Richter M., Godement P. \& Pasquale E.B. (2006) Eph receptors inactivate RRas through different mechanisms to achieve cell repulsion. J Cell Sci 119:12441254.

Dalva M.B., Takasu M.A., Lin M.Z., Shamah S.M., Hu L., Gale N.W. \& Greenberg M.E. (2000) EphB receptors interact with NMDA receptors and regulate excitatory synapse formation. Cell 103:945-956.

Daniel T.O., Stein E., Cerretti D.P., St John P.L., Robert B. \& Abrahamson D.R. (1996) ELK and LERK-2 in developing kidney and microvascular endothelial assembly. Kidney Int Suppl 57:S73-81.

Davis S., Gale N.W., Aldrich T.H., Maisonpierre P.C., Lhotak V., Pawson T., Goldfarb M. \& Yancopoulos G.D. (1994) Ligands for EPH-related receptor tyrosine kinases that require membrane attachment or clustering for activity. Science 266:816-819.

Davy A., Aubin J. \& Soriano P. (2004) Ephrin-B1 forward and reverse signaling are required during mouse development. Genes Dev 18:572-583.

Davy A., Gale N.W., Murray E.W., Klinghoffer R.A., Soriano P., Feuerstein C. \& Robbins S.M. (1999) Compartmentalized signaling by GPI-anchored ephrin-A5 requires the Fyn tyrosine kinase to regulate cellular adhesion. Genes Dev 13:3125-3135.

Davy A. \& Soriano P. (2005) Ephrin signaling in vivo: look both ways. Dev Dyn 232:110. 
Depaepe V., Suarez-Gonzalez N., Dufour A., Passante L., Gorski J.A., Jones K.R., Ledent C. \& Vanderhaeghen P. (2005) Ephrin signalling controls brain size by regulating apoptosis of neural progenitors. Nature 435:1244-1250.

Dergham P., Ellezam B., Essagian C., Avedissian H., Lubell W.D. \& McKerracher L. (2002) Rho signaling pathway targeted to promote spinal cord repair. J Neurosci 22:6570-6577.

Derry J.M., Ochs H.D. \& Francke U. (1994) Isolation of a novel gene mutated in Wiskott-Aldrich syndrome. Cell 78:635-644.

Desai C.J., Garrity P.A., Keshishian H., Zipursky S.L. \& Zinn K. (1999) The Drosophila $\mathrm{SH} 2-\mathrm{SH} 3$ adapter protein Dock is expressed in embryonic axons and facilitates synapse formation by the RP3 motoneuron. Development 126:1527-1535.

Dickson B.J. (2001) Rho GTPases in growth cone guidance. Curr Opin Neurobiol 11:103-110.

Dodelet V.C., Pazzagli C., Zisch A.H., Hauser C.A. \& Pasquale E.B. (1999) A novel signaling intermediate, SHEP1, directly couples Eph receptors to R-Ras and Rap1A. J Biol Chem 274:31941-31946.

Drescher U. (1997) The Eph family in the patterning of neural development. Curr Biol 7:R799-807.

Drescher U. (2002) Eph family functions from an evolutionary perspective. Curr Opin Genet Dev 12:397-402.

Drescher U., Bonhoeffer F. \& Muller B.K. (1997) The Eph family in retinal axon guidance. Curr Opin Neurobiol 7:75-80.

Drescher U., Kremoser C., Handwerker C., Loschinger J., Noda M. \& Bonhoeffer F. (1995) In vitro guidance of retinal ganglion cell axons by RAGS, a $25 \mathrm{kDa}$ tectal protein related to ligands for Eph receptor tyrosine kinases. Cell 82:359-370.

Dutting D., Handwerker C. \& Drescher U. (1999) Topographic targeting and pathfinding errors of retinal axons following overexpression of ephrinA ligands on retinal ganglion cell axons. Dev Biol 216:297-311.

Duxbury M.S., Ito H., Zinner M.J., Ashley S.W. \& Whang E.E. (2004) Ligation of EphA2 by Ephrin A1-Fc inhibits pancreatic adenocarcinoma cellular invasiveness. Biochem Biophys Res Commun 320:1096-1102.

Easty D.J., Hill S.P., Hsu M.Y., Fallowfield M.E., Florenes V.A., Herlyn M. \& Bennett D.C. (1999) Up-regulation of ephrin-A1 during melanoma progression. Int $\mathbf{J}$ Cancer 84:494-501.

Eberhart J., Barr J., O'Connell S., Flagg A., Swartz M.E., Cramer K.S., Tosney K.W., Pasquale E.B. \& Krull C.E. (2004) Ephrin-A5 exerts positive or inhibitory effects on distinct subsets of EphA4-positive motor neurons. Journal of Neuroscience 24:1070-1078.

Edwards D.C., Sanders L.C., Bokoch G.M. \& Gill G.N. (1999) Activation of LIM-kinase by Pak1 couples Rac/Cdc42 GTPase signalling to actin cytoskeletal dynamics.[see comment]. Nature Cell Biology 1:253-259.

Egan S.E., Giddings B.W., Brooks M.W., Buday L., Sizeland A.M. \& Weinberg R.A. (1993) Association of Sos Ras exchange protein with Grb2 is implicated in tyrosine kinase signal transduction and transformation. Nature 363:45-51.

Ellis C., Kasmi F., Ganju P., Walls E., Panayotou G. \& Reith A.D. (1996) A juxtamembrane autophosphorylation site in the Eph family receptor tyrosine 
kinase, Sek, mediates high affinity interaction with p59fyn. Oncogene 12:17271736.

Elowe S., Holland S.J., Kulkarni S. \& Pawson T. (2001) Downregulation of the Rasmitogen-activated protein kinase pathway by the EphB2 receptor tyrosine kinase is required for ephrin-induced neurite retraction. Mol Cell Biol 21:7429-7441.

Eriksson A., Rorsman C., Ernlund A., Claesson-Welsh L. \& Heldin C.H. (1992) Ligandinduced homo- and hetero-dimerization of platelet-derived growth factor alphaand beta-receptors in intact cells. Growth Factors 6:1-14.

Ernst A.F., Jurney W.M. \& McLoon S.C. (1998) Mechanisms involved in development of retinotectal connections: roles of Eph receptor tyrosine kinases, NMDA receptors and nitric oxide. Prog Brain Res 118:115-131.

Ethell I.M., Irie F., Kalo M.S., Couchman J.R., Pasquale E.B. \& Yamaguchi Y. (2001) EphB/syndecan-2 signaling in dendritic spine morphogenesis. Neuron 31:10011013.

Fang W.B., Brantley-Sieders D.M., Parker M.A., Reith A.D. \& Chen J. (2005) A kinasedependent role for EphA2 receptor in promoting tumor growth and metastasis. Oncogene 24:7859-7868.

Feldheim D.A., Kim Y.I., Bergemann A.D., Frisen J., Barbacid M. \& Flanagan J.G. (2000) Genetic analysis of ephrin-A2 and ephrin-A5 shows their requirement in multiple aspects of retinocollicular mapping. Neuron 25:563-574.

Fields S. \& Song O. (1989) A novel genetic system to detect protein-protein interactions. Nature 340:245-246.

Finkelstein L.D. \& Shimizu Y. (2000) Role of phosphoinositide 3-kinase and the Cbl adaptor protein in coupling the alpha4beta1 integrin to mitogen-activated protein kinase signalling. Biochem J 345 Pt 2:385-392.

Flanagan J.G. \& Vanderhaeghen P. (1998) The ephrins and Eph receptors in neural development. Annu Rev Neurosci 21:309-345.

Forscher P., Kaczmarek L.K., Buchanan J.A. \& Smith S.J. (1987) Cyclic AMP induces changes in distribution and transport of organelles within growth cones of Aplysia bag cell neurons. J Neurosci 7:3600-3611.

Fournier A.E., Takizawa B.T. \& Strittmatter S.M. (2003) Rho kinase inhibition enhances axonal regeneration in the injured CNS. J Neurosci 23:1416-1423.

Frese S., Schubert W.D., Findeis A.C., Marquardt T., Roske Y.S., Stradal T.E. \& Heinz D.W. (2006) The phosphotyrosine peptide binding specificity of Nck1 and Nck2 Src homology 2 domains. J Biol Chem 281:18236-18245.

Friedman G.C. \& O'Leary D.D. (1996) Eph receptor tyrosine kinases and their ligands in neural development. Curr Opin Neurobiol 6:127-133.

Frisen J., Holmberg J. \& Barbacid M. (1999) Ephrins and their Eph receptors: multitalented directors of embryonic development. Embo J 18:5159-5165.

Frisen J., Yates P.A., McLaughlin T., Friedman G.C., O'Leary D.D. \& Barbacid M. (1998) Ephrin-A5 (AL-1/RAGS) is essential for proper retinal axon guidance and topographic mapping in the mammalian visual system. Neuron 20:235-243.

Gale N.W., Flenniken A., Compton D.C., Jenkins N., Copeland N.G., Gilbert D.J., Davis S., Wilkinson D.G. \& Yancopoulos G.D. (1996a) Elk-L3, a novel transmembrane ligand for the Eph family of receptor tyrosine kinases, expressed in embryonic floor plate, roof plate and hindbrain segments. Oncogene 13:1343-1352. 
Gale N.W., Holland S.J., Valenzuela D.M., Flenniken A., Pan L., Ryan T.E., Henkemeyer M., Strebhardt K., Hirai H., Wilkinson D.G., Pawson T., Davis S. \& Yancopoulos G.D. (1996b) Eph receptors and ligands comprise two major specificity subclasses and are reciprocally compartmentalized during embryogenesis. Neuron 17:9-19.

Galisteo M.L., Chernoff J., Su Y.C., Skolnik E.Y. \& Schlessinger J. (1996) The adaptor protein Nck links receptor tyrosine kinases with the serine-threonine kinase Pak1. Journal of Biological Chemistry 271:20997-21000.

Gao P.P., Sun C.H., Zhou X.F., DiCicco-Bloom E. \& Zhou R. (2000) Ephrins stimulate or inhibit neurite outgrowth and survival as a function of neuronal cell type [In Process Citation]. J Neurosci Res 60:427-436.

Gao P.P., Yue Y., Cerretti D.P., Dreyfus C. \& Zhou R. (1999) Ephrin-dependent growth and pruning of hippocampal axons. Proc Natl Acad Sci U S A 96:4073-4077.

Gao P.P., Zhang J.H., Yokoyama M., Racey B., Dreyfus C.F., Black I.B. \& Zhou R. (1996) Regulation of topographic projection in the brain: Elf-1 in the hippocamposeptal system. Proc Natl Acad Sci U S A 93:11161-11166.

Garrity P.A., Rao Y., Salecker I., McGlade J., Pawson T. \& Zipursky S.L. (1996) Drosophila photoreceptor axon guidance and targeting requires the dreadlocks SH2/SH3 adapter protein. Cell 85:639-650.

Gerety S.S., Wang H.U., Chen Z.F. \& Anderson D.J. (1999) Symmetrical mutant phenotypes of the receptor EphB4 and its specific transmembrane ligand ephrinB2 in cardiovascular development. Mol Cell 4:403-414.

Goldberg D.J. \& Burmeister D.W. (1986) Stages in axon formation: observations of growth of Aplysia axons in culture using video-enhanced contrast-differential interference contrast microscopy. J Cell Biol 103:1921-1931.

Grunwald I.C., Korte M., Wolfer D., Wilkinson G.A., Unsicker K., Lipp H.P., Bonhoeffer T. \& Klein R. (2001a) Kinase-Independent Requirement of EphB2 Receptors in Hippocampal Synaptic Plasticity. Neuron 32:1027-1040.

Grunwald I.C., Korte M., Wolfer D., Wilkinson G.A., Unsicker K., Lipp H.P., Bonhoeffer T. \& Klein R. (2001b) Kinase-independent requirement of EphB2 receptors in hippocampal synaptic plasticity. Neuron 32:1027-1040.

Gu C. \& Park S. (2001) The EphA8 receptor regulates integrin activity through p110gamma phosphatidylinositol-3 kinase in a tyrosine kinase activityindependent manner. Mol Cell Biol 21:4579-4597.

Guan J.L. (1997) Focal adhesion kinase in integrin signaling. Matrix Biol 16:195-200.

Gupta R.W. \& Mayer B.J. (1998) Dominant-negative mutants of the SH2/SH3 adapters Nck and Grb2 inhibit MAP kinase activation and mesoderm-specific gene induction by eFGF in Xenopus. Oncogene 17:2155-2165.

Hafner C., Becker B., Landthaler M. \& Vogt T. (2006) Expression profile of Eph receptors and ephrin ligands in human skin and downregulation of EphA1 in nonmelanoma skin cancer. Mod Pathol 19:1369-1377.

Hall H., Williams E.J., Moore S.E., Walsh F.S., Prochiantz A. \& Doherty P. (1996) Inhibition of FGF-stimulated phosphatidylinositol hydrolysis and neurite outgrowth by a cell-membrane permeable phosphopeptide. Curr Biol 6:580-587. 
Hamada K., Oike Y., Ito Y., Maekawa H., Miyata K., Shimomura T. \& Suda T. (2003) Distinct roles of ephrin-B2 forward and EphB4 reverse signaling in endothelial cells. Arterioscler Thromb Vasc Biol 23:190-197.

Hansen M.J., Dallal G.E. \& Flanagan J.G. (2004) Retinal axon response to ephrin-as shows a graded, concentration-dependent transition from growth promotion to inhibition. Neuron 42:717-730.

Hattori M., Osterfield M. \& Flanagan J.G. (2000) Regulated cleavage of a contactmediated axon repellent. Science 289:1360-1365.

He T.C., Zhou S., da Costa L.T., Yu J., Kinzler K.W. \& Vogelstein B. (1998) A simplified system for generating recombinant adenoviruses. Proc Natl Acad Sci U S A 95:2509-2514.

Henderson J.T., Georgiou J., Jia Z., Robertson J., Elowe S., Roder J.C. \& Pawson T. (2001) The Receptor Tyrosine Kinase EphB2 Regulates NMDA-Dependent Synaptic Function. Neuron 32:1041-1056.

Henkemeyer M., Marengere L.E., McGlade J., Olivier J.P., Conlon R.A., Holmyard D.P., Letwin K. \& Pawson T. (1994) Immunolocalization of the Nuk receptor tyrosine kinase suggests roles in segmental patterning of the brain and axonogenesis. Oncogene 9:1001-1014.

Henkemeyer M., Orioli D., Henderson J.T., Saxton T.M., Roder J., Pawson T. \& Klein R. (1996) Nuk controls pathfinding of commissural axons in the mammalian central nervous system. Cell 86:35-46.

Hernandez S.E., Krishnaswami M., Miller A.L. \& Koleske A.J. (2004) How do Abl family kinases regulate cell shape and movement? Trends Cell Biol 14:36-44.

Heroult M., Schaffner F. \& Augustin H.G. (2006) Eph receptor and ephrin ligandmediated interactions during angiogenesis and tumor progression. Exp Cell Res 312:642-650.

Himanen J.P., Chumley M.J., Lackmann M., Li C., Barton W.A., Jeffrey P.D., Vearing C., Geleick D., Feldheim D.A., Boyd A.W., Henkemeyer M. \& Nikolov D.B. (2004) Repelling class discrimination: ephrin-A5 binds to and activates EphB2 receptor signaling. Nat Neurosci 7:501-509. Epub 2004 Apr 2025.

Himanen J.P., Henkemeyer M. \& Nikolov D.B. (1998) Crystal structure of the ligandbinding domain of the receptor tyrosine kinase EphB2. Nature 396:486-491.

Himanen J.P. \& Nikolov D.B. (2003a) Eph receptors and ephrins. Int J Biochem Cell Biol 35:130-134.

Himanen J.P. \& Nikolov D.B. (2003b) Eph signaling: a structural view. Trends Neurosci 26:46-51.

Himanen J.P., Rajashankar K.R., Lackmann M., Cowan C.A., Henkemeyer M. \& Nikolov D.B. (2001) Crystal structure of an Eph receptor-ephrin complex. Nature 414:933-938.

Hindges R., McLaughlin T., Genoud N., Henkemeyer M. \& O'Leary D. (2002a) EphB Forward Signaling Controls Directional Branch Extension and Arborization Required for Dorsal-Ventral Retinotopic Mapping. Neuron 35:475.

Hindges R., McLaughlin T., Genoud N., Henkemeyer M. \& O'Leary D.D. (2002b) EphB forward signaling controls directional branch extension and arborization required for dorsal-ventral retinotopic mapping. Neuron 35:475-487. 
Hing H., Xiao J., Harden N., Lim L. \& Zipursky S.L. (1999) Pak functions downstream of Dock to regulate photoreceptor axon guidance in Drosophila. Cell 97:853-863.

Hirai H., Maru Y., Hagiwara K., Nishida J. \& Takaku F. (1987) A novel putative tyrosine kinase receptor encoded by the eph gene. Science 238:1717-1720.

Hock B., Bohme B., Karn T., Feller S., Rubsamen-Waigmann H. \& Strebhardt K. (1998a) Tyrosine-614, the major autophosphorylation site of the receptor tyrosine kinase HEK2, functions as multi-docking site for SH2-domain mediated interactions. Oncogene 17:255-260.

Hock B., Bohme B., Karn T., Yamamoto T., Kaibuchi K., Holtrich U., Holland S., Pawson T., Rubsamen-Waigmann H. \& Strebhardt K. (1998b) PDZ-domainmediated interaction of the Eph-related receptor tyrosine kinase EphB3 and the ras-binding protein AF6 depends on the kinase activity of the receptor. Proc Natl Acad Sci U S A 95:9779-9784.

Holden P.H., Asopa V., Robertson A.G., Clarke A.R., Tyler S., Bennett G.S., Brain S.D., Wilcock G.K., Allen S.J., Smith S.K. \& Dawbarn D. (1997) Immunoglobulin-like domains define the nerve growth factor binding site of the TrkA receptor. Nat Biotechnol 15:668-672.

Holland S.J., Gale N.W., Gish G.D., Roth R.A., Songyang Z., Cantley L.C., Henkemeyer M., Yancopoulos G.D. \& Pawson T. (1997) Juxtamembrane tyrosine residues couple the Eph family receptor EphB2/Nuk to specific SH2 domain proteins in neuronal cells. Embo J 16:3877-3888.

Holland S.J., Gale N.W., Mbamalu G., Yancopoulos G.D., Henkemeyer M. \& Pawson T. (1996) Bidirectional signalling through the EPH-family receptor Nuk and its transmembrane ligands. Nature 383:722-725.

Holmberg J., Armulik A., Senti K.A., Edoff K., Spalding K., Momma S., Cassidy R., Flanagan J.G. \& Frisen J. (2005) Ephrin-A2 reverse signaling negatively regulates neural progenitor proliferation and neurogenesis. Genes Dev 19:462-471.

Holmberg J., Clarke D.L. \& Frisen J. (2000) Regulation of repulsion versus adhesion by different splice forms of an Eph receptor. Nature 408:203-206.

Holmberg J. \& Frisen J. (2002) Ephrins are not only unattractive. Trends Neurosci 25:239-243.

Howe A.K., Aplin A.E. \& Juliano R.L. (2002) Anchorage-dependent ERK signaling-mechanisms and consequences. Curr Opin Genet Dev 12:30-35.

Hu Q., Milfay D. \& Williams L.T. (1995) Binding of NCK to SOS and activation of rasdependent gene expression. Mol Cell Biol 15:1169-1174.

Huang C., Ni Y., Wang T., Gao Y., Haudenschild C.C. \& Zhan X. (1997) Downregulation of the filamentous actin cross-linking activity of cortactin by Srcmediated tyrosine phosphorylation. J Biol Chem 272:13911-13915.

Hummel T., Leifker K. \& Klambt C. (2000) The Drosophila HEM-2/NAP1 homolog KETTE controls axonal pathfinding and cytoskeletal organization. Genes Dev 14:863-873.

Huot J. (2004) Ephrin signaling in axon guidance. Prog Neuropsychopharmacol Biol Psychiatry 28:813-818.

Huynh-Do U., Stein E., Lane A.A., Liu H., Cerretti D.P. \& Daniel T.O. (1999) Surface densities of ephrin-B1 determine EphB1-coupled activation of cell attachment through alphavbeta3 and alpha5beta1 integrins. Embo J 18:2165-2173. 
Ibarra N., Pollitt A. \& Insall R.H. (2005) Regulation of actin assembly by SCAR/WAVE proteins. Biochem Soc Trans 33:1243-1246.

Irie F., Okuno M., Pasquale E.B. \& Yamaguchi Y. (2005) EphrinB-EphB signalling regulates clathrin-mediated endocytosis through tyrosine phosphorylation of synaptojanin 1. Nat Cell Biol 7:501-509.

Jendraschak E. \& Sage E.H. (1996) Regulation of angiogenesis by SPARC and angiostatin: implications for tumor cell biology. Semin Cancer Biol 7:139-146.

Kahn R.A., Volpicelli-Daley L., Bowzard B., Shrivastava-Ranjan P., Li Y., Zhou C. \& Cunningham L. (2005) Arf family GTPases: roles in membrane traffic and microtubule dynamics. Biochem Soc Trans 33:1269-1272.

Kalo M.S. \& Pasquale E.B. (1999) Signal transfer by Eph receptors. Cell Tissue Res 298:1-9.

Kantardzhieva A., Alexeeva S., Versteeg I. \& Wijnholds J. (2006) MPP3 is recruited to the MPP5 protein scaffold at the retinal outer limiting membrane. Febs $\mathbf{J}$ 273:1152-1165.

Karanian D.A., Brown Q.B., Makriyannis A. \& Bahr B.A. (2005) Blocking cannabinoid activation of FAK and ERK1/2 compromises synaptic integrity in hippocampus. Eur J Pharmacol 508:47-56.

Keegan L., Gill G. \& Ptashne M. (1986) Separation of DNA binding from the transcription-activating function of a eukaryotic regulatory protein. Science 231:699-704.

Kikawa K.D., Vidale D.R., Van Etten R.L. \& Kinch M.S. (2002) Regulation of the EphA2 kinase by the low molecular weight tyrosine phosphatase induces transformation. J Biol Chem 277:39274-39279.

Kilpatrick T.J., Brown A., Lai C., Gassmann M., Goulding M. \& Lemke G. (1996) Expression of the Tyro4/Mek4/Cek4 gene specifically marks a subset of embryonic motor neurons and their muscle targets. Mol Cell Neurosci 7:62-74.

Kinch M.S. \& Carles-Kinch K. (2003) Overexpression and functional alterations of the EphA2 tyrosine kinase in cancer. Clin Exp Metastasis 20:59-68.

Klein R. (2004) Eph/ephrin signaling in morphogenesis, neural development and plasticity. Curr Opin Cell Biol 16:580-589.

Knoll B. \& Drescher U. (2002) Ephrin-As as receptors in topographic projections. Trends Neurosci 25:145-149.

Knoll B., Isenmann S., Kilic E., Walkenhorst J., Engel S., Wehinger J., Bahr M. \& Drescher U. (2001) Graded expression patterns of ephrin-As in the superior colliculus after lesion of the adult mouse optic nerve. Mech Dev 106:119-127.

Koleske A.J., Gifford A.M., Scott M.L., Nee M., Bronson R.T., Miczek K.A. \& Baltimore D. (1998) Essential roles for the Abl and Arg tyrosine kinases in neurulation. Neuron 21:1259-1272.

Krull C.E., Lansford R., Gale N.W., Collazo A., Marcelle C., Yancopoulos G.D., Fraser S.E. \& Bronner-Fraser M. (1997) Interactions of Eph-related receptors and ligands confer rostrocaudal pattern to trunk neural crest migration. Curr Biol 7:571-580.

Kullander K., Croll S.D., Zimmer M., Pan L., McClain J., Hughes V., Zabski S., DeChiara T.M., Klein R., Yancopoulos G.D. \& Gale N.W. (2001) Ephrin-B3 is 
the midline barrier that prevents corticospinal tract axons from recrossing, allowing for unilateral motor control. Genes Dev 15:877-888.

Kullander K. \& Klein R. (2002) Mechanisms and functions of Eph and ephrin signalling. Nat Rev Mol Cell Biol 3:475-486.

Kunapuli P., Kasyapa C.S., Hawthorn L. \& Cowell J.K. (2004) LGI1, a putative tumor metastasis suppressor gene, controls in vitro invasiveness and expression of matrix metalloproteinases in glioma cells through the ERK1/2 pathway. J Biol Chem 279:23151-23157.

Kyurkchiev S., Yeger H., Bleau A.M. \& Perbal B. (2004) Potential cellular conformations of the CCN3(NOV) protein. Cell Commun Signal 2:9.

Lamprecht R., Farb C.R. \& LeDoux J.E. (2002) Fear memory formation involves p190 RhoGAP and ROCK proteins through a GRB2-mediated complex. Neuron 36:727-738.

Lawrenson I.D., Wimmer-Kleikamp S.H., Lock P., Schoenwaelder S.M., Down M., Boyd A.W., Alewood P.F. \& Lackmann M. (2002a) Ephrin-A5 induces rounding, blebbing and de-adhesion of EphA3- expressing 293T and melanoma cells by CrkII and Rho-mediated signalling. J Cell Sci 115:1059-1072.

Lawrenson I.D., Wimmer-Kleikamp S.H., Lock P., Schoenwaelder S.M., Down M., Boyd A.W., Alewood P.F. \& Lackmann M. (2002b) Ephrin-A5 induces rounding, blebbing and de-adhesion of EphA3-expressing 293T and melanoma cells by CrkII and Rho-mediated signalling. J Cell Sci 115:1059-1072.

Li W., Fan J. \& Woodley D.T. (2001) Nck/Dock: an adapter between cell surface receptors and the actin cytoskeleton. Oncogene 20:6403-6417.

Li W., Hu P., Skolnik E.Y., Ullrich A. \& Schlessinger J. (1992) The SH2 and SH3 domain-containing Nck protein is oncogenic and a common target for phosphorylation by different surface receptors. Mol Cell Biol 12:5824-5833.

Li X., Meriane M., Triki I., Shekarabi M., Kennedy T.E., Larose L. \& Lamarche-Vane N. (2002a) The adaptor protein Nck-1 couples the netrin-1 receptor DCC (deleted in colorectal cancer) to the activation of the small GTPase Rac1 through an atypical mechanism. Journal of Biological Chemistry 277:37788-37797.

Li X., Meriane M., Triki I., Shekarabi M., Kennedy T.E., Larose L. \& Lamarche-Vane N. (2002b) The adaptor protein Nck-1 couples the netrin-1 receptor DCC (deleted in colorectal cancer) to the activation of the small GTPase Rac1 through an atypical mechanism. J Biol Chem 277:37788-37797.

Lin D., Gish G.D., Songyang Z. \& Pawson T. (1999) The carboxyl terminus of B class ephrins constitutes a PDZ domain binding motif. J Biol Chem 274:3726-3733.

Lu Q., Sun E.E. \& Flanagan J.G. (2004) Analysis of PDZ-RGS3 function in ephrin-B reverse signaling. Methods Enzymol 390:120-128.

Lu Q., Sun E.E., Klein R.S. \& Flanagan J.G. (2001) Ephrin-B reverse signaling is mediated by a novel PDZ-RGS protein and selectively inhibits G protein-coupled chemoattraction. Cell 105:69-79.

Lu W., Katz S., Gupta R. \& Mayer B.J. (1997) Activation of Pak by membrane localization mediated by an SH3 domain from the adaptor protein Nck. Curr Biol 7:85-94. 
Machesky L.M., Mullins R.D., Higgs H.N., Kaiser D.A., Blanchoin L., May R.C., Hall M.E. \& Pollard T.D. (1999) Scar, a WASp-related protein, activates nucleation of actin filaments by the Arp2/3 complex. Proc Natl Acad Sci U S A 96:3739-3744.

Maekawa H., Oike Y., Kanda S., Ito Y., Yamada Y., Kurihara H., Nagai R. \& Suda T. (2003) Ephrin-B2 induces migration of endothelial cells through the phosphatidylinositol-3 kinase pathway and promotes angiogenesis in adult vasculature. Arterioscler Thromb Vasc Biol 23:2008-2014.

Magal E., Holash J.A., Toso R.J., Chang D., Lindberg R.A. \& Pasquale E.B. (1996) B61, a ligand for the Eck receptor protein-tyrosine kinase, exhibits neurotrophic activity in cultures of rat spinal cord neurons. J Neurosci Res 43:735-744.

Mancia F. \& Shapiro L. (2005) ADAM and Eph: how Ephrin-signaling cells become detached. Cell 123:185-187.

Mann F., Miranda E., Weinl C., Harmer E. \& Holt C.E. (2003) B-type Eph receptors and ephrins induce growth cone collapse through distinct intracellular pathways. J Neurobiol 57:323-336.

Mann F., Ray S., Harris W. \& Holt C. (2002) Topographic mapping in dorsoventral axis of the Xenopus retinotectal system depends on signaling through ephrin-B ligands. Neuron 35:461-473.

Marin O., Blanco M.J. \& Nieto M.A. (2001) Differential expression of Eph receptors and ephrins correlates with the formation of topographic projections in primary and secondary visual circuits of the embryonic chick forebrain. Dev Biol 234:289303.

Marston D.J., Dickinson S. \& Nobes C.D. (2003) Rac-dependent trans-endocytosis of ephrinBs regulates Eph-ephrin contact repulsion. Nat Cell Biol 5:879-888.

Marte B.M., Rodriguez-Viciana P., Wennstrom S., Warne P.H. \& Downward J. (1997) R-Ras can activate the phosphoinositide 3-kinase but not the MAP kinase arm of the Ras effector pathways. Curr Biol 7:63-70.

Martinez A. \& Soriano E. (2005) Functions of ephrin/Eph interactions in the development of the nervous system: emphasis on the hippocampal system. Brain Res Brain Res Rev 49:211-226.

Matsuoka H., Obama H., Kelly M.L., Matsui T. \& Nakamoto M. (2005) Biphasic functions of the kinase-defective Ephb6 receptor in cell adhesion and migration. Journal of Biological Chemistry 280:29355-29363.

Mayer B.J. \& Gupta R. (1998) Functions of SH2 and SH3 domains. Curr Top Microbiol Immunol 228:1-22.

McCarty J.H. (1998) The Nck SH2/SH3 adaptor protein: a regulator of multiple intracellular signal transduction events. Bioessays 20:913-921.

McGlade J., Brunkhorst B., Anderson D., Mbamalu G., Settleman J., Dedhar S., RozakisAdcock M., Chen L.B. \& Pawson T. (1993) The N-terminal region of GAP regulates cytoskeletal structure and cell adhesion. Embo J 12:3073-3081.

Meima L., Kljavin I.J., Moran P., Shih A., Winslow J.W. \& Caras I.W. (1997a) AL-1induced growth cone collapse of rat cortical neurons is correlated with REK7 expression and rearrangement of the actin cytoskeleton. Eur J Neurosci 9:177188. 
Meima L., Moran P., Matthews W. \& Caras I.W. (1997b) Lerk2 (ephrin-B1) is a collapsing factor for a subset of cortical growth cones and acts by a mechanism different from AL-1 (ephrin-A5). Mol Cell Neurosci 9:314-328.

Mellitzer G., Xu Q. \& Wilkinson D.G. (1999) Eph receptors and ephrins restrict cell intermingling and communication. Nature 400:77-81.

Mellitzer G., Xu Q. \& Wilkinson D.G. (2000) Control of cell behaviour by signalling through Eph receptors and ephrins. Curr Opin Neurobiol 10:400-408.

Meyer S., Hafner C., Guba M., Flegel S., Geissler E.K., Becker B., Koehl G.E., Orso E., Landthaler M. \& Vogt T. (2005) Ephrin-B2 overexpression enhances integrinmediated ECM-attachment and migration of B16 melanoma cells. Int J Oncol 27:1197-1206.

Miao H., Burnett E., Kinch M., Simon E. \& Wang B. (2000) Activation of EphA2 kinase suppresses integrin function and causes focal-adhesion-kinase dephosphorylation. Nat Cell Biol 2:62-69.

Miao H., Strebhardt K., Pasquale E.B., Shen T.L., Guan J.L. \& Wang B. (2005) Inhibition of integrin-mediated cell adhesion but not directional cell migration requires catalytic activity of EphB3 receptor tyrosine kinase. Role of Rho family small GTPases. J Biol Chem 280:923-932.

Moeller M.L., Shi Y., Reichardt L.F. \& Ethell I.M. (2006) EphB receptors regulate dendritic spine morphogenesis through the recruitment/phosphorylation of focal adhesion kinase and RhoA activation. J Biol Chem 281:1587-1598.

Monschau B., Kremoser C., Ohta K., Tanaka H., Kaneko T., Yamada T., Handwerker C., Hornberger M.R., Loschinger J., Pasquale E.B., Siever D.A., Verderame M.F., Muller B.K., Bonhoeffer F. \& Drescher U. (1997) Shared and distinct functions of RAGS and ELF-1 in guiding retinal axons. Embo J 16:1258-1267.

Moore K.B., Mood K., Daar I.O. \& Moody S.A. (2004) Morphogenetic movements underlying eye field formation require interactions between the FGF and ephrinB1 signaling pathways. Dev Cell 6:55-67.

Muller B., Stahl B. \& Bonhoeffer F. (1990) In vitro experiments on axonal guidance and growth-cone collapse. J Exp Biol 153:29-46.

Murai K.K. \& Pasquale E.B. (2004) Eph receptors, ephrins, and synaptic function. Neuroscientist 10:304-314.

Murai K.K. \& Pasquale E.B. (2005) New exchanges in eph-dependent growth cone dynamics. Neuron 46:161-163.

Nakamoto M. (2000) Eph receptors and ephrins. Int J Biochem Cell Biol 32:7-12.

Nakamoto M. \& Bergemann A.D. (2002) Diverse roles for the Eph family of receptor tyrosine kinases in carcinogenesis. Microsc Res Tech 59:58-67.

Navolanic P.M., Steelman L.S. \& McCubrey J.A. (2003) EGFR family signaling and its association with breast cancer development and resistance to chemotherapy (Review). Int J Oncol 22:237-252.

Nikolic M. (2002) The role of Rho GTPases and associated kinases in regulating neurite outgrowth. Int J Biochem Cell Biol 34:731-745.

Nobes C.D. \& Hall A. (1995) Rho, rac, and cdc42 GTPases regulate the assembly of multimolecular focal complexes associated with actin stress fibers, lamellipodia, and filopodia. Cell 81:53-62. 
Normanno N., De Luca A., Bianco C., Strizzi L., Mancino M., Maiello M.R., Carotenuto A., De Feo G., Caponigro F. \& Salomon D.S. (2006) Epidermal growth factor receptor (EGFR) signaling in cancer. Gene 366:2-16.

Oda Y., Yamamoto H., Takahira T., Kobayashi C., Kawaguchi K., Tateishi N., Nozuka Y., Tamiya S., Tanaka K., Matsuda S., Yokoyama R., Iwamoto Y. \& Tsuneyoshi M. (2005) Frequent alteration of p16(INK4a)/p14(ARF) and p53 pathways in the round cell component of myxoid/round cell liposarcoma: p53 gene alterations and reduced $\mathrm{p} 14(\mathrm{ARF})$ expression both correlate with poor prognosis. J Pathol 207:410-421.

Ogawa K., Pasqualini R., Lindberg R.A., Kain R., Freeman A.L. \& Pasquale E.B. (2000) The ephrin-A1 ligand and its receptor, EphA2, are expressed during tumor neovascularization. Oncogene 19:6043-6052.

Olkkonen V.M., Johansson M., Suchanek M., Yan D., Hynynen R., Ehnholm C., Jauhiainen M., Thiele C. \& Lehto M. (2006) The OSBP-related proteins (ORPs): global sterol sensors for co-ordination of cellular lipid metabolism, membrane trafficking and signalling processes? Biochem Soc Trans 34:389-391.

Orioli D. \& Klein R. (1997) The Eph receptor family: axonal guidance by contact repulsion. Trends Genet 13:354-359.

Otal R., Burgaya F., Frisen J., Soriano E. \& Martinez A. (2006) Ephrin-A5 modulates the topographic mapping and connectivity of commissural axons in murine hippocampus. Neuroscience 141:109-121.

Palmer A., Zimmer M., Erdmann K.S., Eulenburg V., Porthin A., Heumann R., Deutsch U. \& Klein R. (2002) EphrinB phosphorylation and reverse signaling: regulation by Src kinases and PTP-BL phosphatase. Mol Cell 9:725-737.

Pandey A., Duan H. \& Dixit V.M. (1995) Characterization of a novel Src-like adapter protein that associates with the Eck receptor tyrosine kinase. J Biol Chem 270:19201-19204.

Pandey A., Lazar D.F., Saltiel A.R. \& Dixit V.M. (1994) Activation of the Eck receptor protein tyrosine kinase stimulates phosphatidylinositol 3-kinase activity. J Biol Chem 269:30154-30157.

Park D. \& Rhee S.G. (1992) Phosphorylation of Nck in response to a variety of receptors, phorbol myristate acetate, and cyclic AMP. Mol Cell Biol 12:5816-5823.

Parri M., Buricchi F., Taddei M.L., Giannoni E., Raugei G., Ramponi G. \& Chiarugi P. (2005) EphrinA1 repulsive response is regulated by an EphA2 tyrosine phosphatase. J Biol Chem 280:34008-34018.

Parsons J.T. \& Parsons S.J. (1997) Src family protein tyrosine kinases: cooperating with growth factor and adhesion signaling pathways. Curr Opin Cell Biol 9:187-192.

Pascall J.C. \& Brown K.D. (2004) Intramembrane cleavage of ephrinB3 by the human rhomboid family protease, RHBDL2. Biochem Biophys Res Commun 317:244252.

Pasquale E.B. (1997) The Eph family of receptors. Curr Opin Cell Biol 9:608-615.

Pasquale E.B. (2004) Eph-ephrin promiscuity is now crystal clear. Nat Neurosci 7:417418.

Pasquale E.B. (2005) Eph receptor signalling casts a wide net on cell behaviour. Nat Rev Mol Cell Biol 6:462-475. 
Pawson T. (1994) SH2 and SH3 domains in signal transduction. Adv Cancer Res 64:87110.

Pawson T. (1995) Protein-tyrosine kinases. Getting down to specifics. Nature 373:477478.

Pei Z., Maloney J.A., Yang L. \& Williamson J.R. (1997) A new function for phospholipase C-gamma1: coupling to the adaptor protein GRB2. Arch Biochem Biophys 345:103-110.

Penzes P., Beeser A., Chernoff J., Schiller M.R., Eipper B.A., Mains R.E. \& Huganir R.L. (2003) Rapid induction of dendritic spine morphogenesis by trans-synaptic ephrinB-EphB receptor activation of the Rho-GEF kalirin. Neuron 37:263-274.

Penzes P., Johnson R.C., Sattler R., Zhang X., Huganir R.L., Kambampati V., Mains R.E. \& Eipper B.A. (2001) The neuronal Rho-GEF Kalirin-7 interacts with PDZ domain-containing proteins and regulates dendritic morphogenesis. Neuron 29:229-242.

Perry R.J. \& Ridgway N.D. (2006) Oxysterol-binding protein and vesicle-associated membrane protein-associated protein are required for sterol-dependent activation of the ceramide transport protein. Mol Biol Cell 17:2604-2616.

Plattner R., Kadlec L., DeMali K.A., Kazlauskas A. \& Pendergast A.M. (1999) c-Abl is activated by growth factors and Src family kinases and has a role in the cellular response to PDGF. Genes Dev 13:2400-2411.

Plattner R., Koleske A.J., Kazlauskas A. \& Pendergast A.M. (2004) Bidirectional signaling links the Abelson kinases to the platelet-derived growth factor receptor. Mol Cell Biol 24:2573-2583.

Pratt R.L. \& Kinch M.S. (2002) Activation of the EphA2 tyrosine kinase stimulates the MAP/ERK kinase signaling cascade. Oncogene 21:7690-7699.

Quilliam L.A., Lambert Q.T., Mickelson-Young L.A., Westwick J.K., Sparks A.B., Kay B.K., Jenkins N.A., Gilbert D.J., Copeland N.G. \& Der C.J. (1996) Isolation of a NCK-associated kinase, PRK2, an SH3-binding protein and potential effector of Rho protein signaling. J Biol Chem 271:28772-28776.

Rao Y. \& Zipursky S.L. (1998) Domain requirements for the Dock adapter protein in growth- cone signaling. Proc Natl Acad Sci U S A 95:2077-2082.

Ribon V., Herrera R., Kay B.K. \& Saltiel A.R. (1998) A role for CAP, a novel, multifunctional Src homology 3 domain-containing protein in formation of actin stress fibers and focal adhesions. J Biol Chem 273:4073-4080.

Riordan S.M., Lidder S., Williams R. \& Skouteris G.G. (2000) The beta-subunit of the hepatocyte growth factor/scatter factor (HGF/SF) receptor phosphorylates and associates with CrkII: expression of CrkII enhances HGF/SF-induced mitogenesis. Biochem J 350 Pt 3:925-932.

Rivera G.M., Antoku S., Gelkop S., Shin N.Y., Hanks S.K., Pawson T. \& Mayer B.J. (2006a) Requirement of Nck adaptors for actin dynamics and cell migration stimulated by platelet-derived growth factor B. Proc Natl Acad Sci U S A 103:9536-9541.

Rivera G.M., Antoku S., Gelkop S., Shin N.Y., Hanks S.K., Pawson T. \& Mayer B.J. (2006b) Requirement of Nck adaptors for actin dynamics and cell migration stimulated by platelet-derived growth factor B. Proceedings of the National Academy of Sciences of the United States of America 103:9536-9541. 
Rivera G.M., Briceno C.A., Takeshima F., Snapper S.B. \& Mayer B.J. (2004) Inducible clustering of membrane-targeted SH3 domains of the adaptor protein Nck triggers localized actin polymerization. Current Biology 14:11-22.

Rivero-Lezcano O.M., Marcilla A., Sameshima J.H. \& Robbins K.C. (1995) WiskottAldrich syndrome protein physically associates with Nck through Src homology 3 domains. Mol Cell Biol 15:5725-5731.

Robinson D.R., Wu Y.M. \& Lin S.F. (2000) The protein tyrosine kinase family of the human genome. Oncogene 19:5548-5557.

Roche S., McGlade J., Jones M., Gish G.D., Pawson T. \& Courtneidge S.A. (1996a) Requirement of phospholipase $\mathrm{C}$ gamma, the tyrosine phosphatase Syp and the adaptor proteins Shc and Nck for PDGF-induced DNA synthesis: evidence for the existence of Ras-dependent and Ras-independent pathways. Embo J 15:49404948.

Roche S., McGlade J., Jones M., Gish G.D., Pawson T. \& Courtneidge S.A. (1996b) Requirement of phospholipase $\mathrm{C}$ gamma, the tyrosine phosphatase Syp and the adaptor proteins Shc and Nck for PDGF-induced DNA synthesis: evidence for the existence of Ras-dependent and Ras-independent pathways. EMBO Journal 15:4940-4948.

Rohatgi R., Nollau P., Ho H.Y., Kirschner M.W. \& Mayer B.J. (2001) Nck and phosphatidylinositol 4,5-bisphosphate synergistically activate actin polymerization through the N-WASP-Arp2/3 pathway. J Biol Chem 276:2644826452.

Roux P.P. \& Blenis J. (2004) ERK and p38 MAPK-activated protein kinases: a family of protein kinases with diverse biological functions. Microbiol Mol Biol Rev 68:320-344.

Ruan W., Pang P. \& Rao Y. (1999) The SH2/SH3 adaptor protein dock interacts with the Ste20-like kinase misshapen in controlling growth cone motility. Neuron 24:595605.

Sahin M., Greer P.L., Lin M.Z., Poucher H., Eberhart J., Schmidt S., Wright T.M., Shamah S.M., O'Connell S., Cowan C.W., Hu L., Goldberg J.L., Debant A., Corfas G., Krull C.E. \& Greenberg M.E. (2005) Eph-dependent tyrosine phosphorylation of ephexin1 modulates growth cone collapse. Neuron 46:191204.

Sajjadi F.G. \& Pasquale E.B. (1993) Five novel avian Eph-related tyrosine kinases are differentially expressed. Oncogene 8:1807-1813.

Sakurai T., Wong E., Drescher U., Tanaka H. \& Jay D.G. (2002) Ephrin-A5 restricts topographically specific arborization in the chick retinotectal projection in vivo. Proc Natl Acad Sci U S A 99:10795-10800.

Saleem A., Datta R., Yuan Z.M., Kharbanda S. \& Kufe D. (1995) Involvement of stressactivated protein kinase in the cellular response to 1-beta-Darabinofuranosylcytosine and other DNA-damaging agents. Cell Growth Differ 6:1651-1658.

Salgia R., Li J.L., Ewaniuk D.S., Pear W., Pisick E., Burky S.A., Ernst T., Sattler M., Chen L.B. \& Griffin J.D. (1997) BCR/ABL induces multiple abnormalities of cytoskeletal function. J Clin Invest 100:46-57. 
Samarin S.N. (2005) WASP family proteins act between cytoskeleton and cellular signaling pathways. Biochemistry (Mosc) 70:1305-1309.

Sanders L.C., Matsumura F., Bokoch G.M. \& de Lanerolle P. (1999) Inhibition of myosin light chain kinase by p21-activated kinase. Science 283:2083-2085.

Sasaki E., Hikono H., Kaku Y., Kuwana T., Naito M. \& Sakurai M. (2003) ephA9, a novel avian receptor tyrosine kinase gene. Gene 316:103-110.

Savina A., Fader C.M., Damiani M.T. \& Colombo M.I. (2005) Rab11 promotes docking and fusion of multivesicular bodies in a calcium-dependent manner. Traffic 6:131-143.

Schlaepfer D.D. \& Hunter T. (1998) Integrin signalling and tyrosine phosphorylation: just the FAKs? Trends Cell Biol 8:151-157.

Schmucker D. \& Zipursky S.L. (2001) Signaling downstream of Eph receptors and ephrin ligands. Cell 105:701-704.

Shamah S.M., Lin M.Z., Goldberg J.L., Estrach S., Sahin M., Hu L., Bazalakova M., Neve R.L., Corfas G., Debant A. \& Greenberg M.E. (2001a) EphA receptors regulate growth cone dynamics through the novel guanine nucleotide exchange factor ephexin. Cell 105:233-244.

Shamah S.M., Lin M.Z., Goldberg J.L., Estrach S., Sahin M., Hu L., Bazalakova M., Neve R.L., Corfas G., Debant A. \& Greenberg M.E. (2001b) EphA receptors regulate growth cone dynamics through the novel guanine nucleotide exchange factor ephexin. Cell 105:233-244.

Sharfe N., Freywald A., Toro A. \& Roifman C.M. (2003) Ephrin-A1 induces c-Cbl phosphorylation and EphA receptor down-regulation in T cells. J Immunol 170:6024-6032.

Shi Y., Alin K. \& Goff S.P. (1995) Abl-interactor-1, a novel SH3 protein binding to the carboxy-terminal portion of the Abl protein, suppresses v-abl transforming activity. Genes Dev 9:2583-2597.

Shimizu Y., Irani A.M., Brown E.J., Ashman L.K. \& Schwartz L.B. (1995) Human mast cells derived from fetal liver cells cultured with stem cell factor express a functional CD51/CD61 (alpha v beta 3) integrin. Blood 86:930-939.

Shinkaruk S., Bayle M., Lain G. \& Deleris G. (2003) Vascular endothelial cell growth factor (VEGF), an emerging target for cancer chemotherapy. Curr Med Chem Anticancer Agents 3:95-117.

Shintani T., Ihara M., Sakuta H., Takahashi H., Watakabe I. \& Noda M. (2006) Eph receptors are negatively controlled by protein tyrosine phosphatase receptor type O. Nat Neurosci 9:761-769.

Shurin M.R., Esche C. \& Lotze M.T. (1998) FLT3: receptor and ligand. Biology and potential clinical application. Cytokine Growth Factor Rev 9:37-48.

Simons K. \& Toomre D. (2000) Lipid rafts and signal transduction. Nat Rev Mol Cell Biol 1:31-39.

Smith A., Robinson V., Patel K. \& Wilkinson D.G. (1997) The EphA4 and EphB1 receptor tyrosine kinases and ephrin-B2 ligand regulate targeted migration of branchial neural crest cells. Curr Biol 7:561-570.

Smith F.M., Vearing C., Lackmann M., Treutlein H., Himanen J., Chen K., Saul A., Nikolov D. \& Boyd A.W. (2004) Dissecting the EphA3/Ephrin-A5 interactions using a novel functional mutagenesis screen. J Biol Chem 279:9522-9531. 
Songyang Z., Shoelson S.E., Chaudhuri M., Gish G., Pawson T., Haser W.G., King F., Roberts T., Ratnofsky S., Lechleider R.J. \& et al. (1993a) SH2 domains recognize specific phosphopeptide sequences. Cell 72:767-778.

Songyang Z., Shoelson S.E., Chaudhuri M., Gish G., Pawson T., Haser W.G., King F., Roberts T., Ratnofsky S., Lechleider R.J. \& et al. (1993b) SH2 domains recognize specific phosphopeptide sequences. Cell 72:767-778.

Sperry R.W. (1950) Neural basis of the spontaneous optokinetic response produced by visual inversion. J Comp Physiol Psychol 43:482-489.

Sperry R.W. (1951) Regulative factors in the orderly growth of neural circuits. Growth 15:63-87.

Stein E., Cerretti D.P. \& Daniel T.O. (1996) Ligand activation of ELK receptor tyrosine kinase promotes its association with Grb10 and Grb2 in vascular endothelial cells. J Biol Chem 271:23588-23593.

Stein E., Huynh-Do U., Lane A.A., Cerretti D.P. \& Daniel T.O. (1998a) Nck recruitment to Eph receptor, EphB1/ELK, couples ligand activation to c-Jun kinase. J Biol Chem 273:1303-1308.

Stein E., Lane A.A., Cerretti D.P., Schoecklmann H.O., Schroff A.D., Van Etten R.L. \& Daniel T.O. (1998b) Eph receptors discriminate specific ligand oligomers to determine alternative signaling complexes, attachment, and assembly responses. Genes Dev 12:667-678.

Stein M.P., Dong J. \& Wandinger-Ness A. (2003) Rab proteins and endocytic trafficking: potential targets for therapeutic intervention. Adv Drug Deliv Rev 55:1421-1437.

Stoletov K.V., Gong C. \& Terman B.I. (2004) Nck and Crk mediate distinct VEGFinduced signaling pathways that serve overlapping functions in focal adhesion turnover and integrin activation. Exp Cell Res 295:258-268.

Su Y.C., Maurel-Zaffran C., Treisman J.E. \& Skolnik E.Y. (2000) The Ste20 kinase misshapen regulates both photoreceptor axon targeting and dorsal closure, acting downstream of distinct signals. Mol Cell Biol 20:4736-4744.

Su Y.C., Treisman J.E. \& Skolnik E.Y. (1998) The Drosophila Ste20-related kinase misshapen is required for embryonic dorsal closure and acts through a JNK MAPK module on an evolutionarily conserved signaling pathway. Genes Dev 12:2371-2380.

Su Z., Xu P. \& Ni F. (2004) Single phosphorylation of Tyr304 in the cytoplasmic tail of ephrin B2 confers high-affinity and bifunctional binding to both the $\mathrm{SH} 2$ domain of Grb4 and the PDZ domain of the PDZ-RGS3 protein. Eur J Biochem 271:1725-1736.

Suenobu S., Takakura N., Inada T., Yamada Y., Yuasa H., Zhang X.Q., Sakano S., Oike Y. \& Suda T. (2002) A role of EphB4 receptor and its ligand, ephrin-B2, in erythropoiesis. Biochem Biophys Res Commun 293:1124-1131.

Surawska H., Ma P.C. \& Salgia R. (2004) The role of ephrins and Eph receptors in cancer. Cytokine Growth Factor Rev 15:419-433.

Takasu M.A., Dalva M.B., Zigmond R.E. \& Greenberg M.E. (2002a) Modulation of NMDA receptor-dependent calcium influx and gene expression through EphB receptors. Science 295:491-495. 
Takasu M.A., Dalva M.B., Zigmond R.E. \& Greenberg M.E. (2002b) Modulation of NMDA receptor-dependent calcium influx and gene expression through EphB receptors. Science 295:491-495.

Takenawa T. \& Suetsugu S. (2007) The WASP-WAVE protein network: connecting the membrane to the cytoskeleton. Nat Rev Mol Cell Biol 8:37-48.

Tanaka M., Gupta R. \& Mayer B.J. (1995a) Differential inhibition of signaling pathways by dominant-negative SH2/SH3 adapter proteins. Mol Cell Biol 15:6829-6837.

Tanaka M., Gupta R. \& Mayer B.J. (1995b) Differential inhibition of signaling pathways by dominant-negative SH2/SH3 adapter proteins. Molecular \& Cellular Biology 15:6829-6837.

Tanaka M., Kamo T., Ota S. \& Sugimura H. (2003) Association of Dishevelled with Eph tyrosine kinase receptor and ephrin mediates cell repulsion. Embo J 22:847-858.

Tanaka M., Ohashi R., Nakamura R., Shinmura K., Kamo T., Sakai R. \& Sugimura H. (2004) Tiam1 mediates neurite outgrowth induced by ephrin-B1 and EphA2. Embo J 23:1075-1088.

Tang J., Feng G.S. \& Li W. (1997) Induced direct binding of the adapter protein Nck to the GTPase-activating protein-associated protein $\mathrm{p} 62$ by epidermal growth factor. Oncogene 15:1823-1832.

Tang X.X., Zhao H., Robinson M.E., Cnaan A., London W., Cohn S.L., Cheung N.K., Brodeur G.M., Evans A.E. \& Ikegaki N. (2000a) Prognostic significance of EPHB6, EFNB2, and EFNB3 expressions in neuroblastoma. Med Pediatr Oncol 35:656-658.

Tang X.X., Zhao H., Robinson M.E., Cohen B., Cnaan A., London W., Cohn S.L., Cheung N.K., Brodeur G.M., Evans A.E. \& Ikegaki N. (2000b) Implications of EPHB6, EFNB2, and EFNB3 expressions in human neuroblastoma. Proc Natl Acad Sci U S A 97:10936-10941.

Tessier-Lavigne M. (1995) Eph receptor tyrosine kinases, axon repulsion, and the development of topographic maps. Cell 82:345-348.

Tessier-Lavigne M. \& Goodman C.S. (1996) The molecular biology of axon guidance. Science 274:1123-1133.

Tong J., Elowe S., Nash P. \& Pawson T. (2003) Manipulation of EphB2 regulatory motifs and $\mathrm{SH} 2$ binding sites switches MAPK signaling and biological activity. J Biol Chem 278:6111-6119.

Torres R., Firestein B.L., Dong H., Staudinger J., Olson E.N., Huganir R.L., Bredt D.S., Gale N.W. \& Yancopoulos G.D. (1998) PDZ proteins bind, cluster, and synaptically colocalize with Eph receptors and their ephrin ligands. Neuron 21:1453-1463.

Tu Y., Li F. \& Wu C. (1998) Nck-2, a novel Src homology2/3-containing adaptor protein that interacts with the LIM-only protein PINCH and components of growth factor receptor kinase-signaling pathways. Mol Biol Cell 9:3367-3382.

Tuynder M., Fiucci G., Prieur S., Lespagnol A., Geant A., Beaucourt S., Duflaut D., Besse S., Susini L., Cavarelli J., Moras D., Amson R. \& Telerman A. (2004) Translationally controlled tumor protein is a target of tumor reversion. Proc Natl Acad Sci U S A 101:15364-15369.

Viala E. \& Pouyssegur J. (2004) Regulation of tumor cell motility by ERK mitogenactivated protein kinases. Ann N Y Acad Sci 1030:208-218. 
Vogt T., Stolz W., Welsh J., Jung B., Kerbel R.S., Kobayashi H., Landthaler M. \& McClelland M. (1998) Overexpression of Lerk-5/Eplg5 messenger RNA: a novel marker for increased tumorigenicity and metastatic potential in human malignant melanomas. Clin Cancer Res 4:791-797.

Wahl S., Barth H., Ciossek T., Aktories K. \& Mueller B.K. (2000) Ephrin-A5 induces collapse of growth cones by activating Rho and Rho kinase. J Cell Biol 149:263270.

Walker-Daniels J., Riese D.J., 2nd \& Kinch M.S. (2002) c-Cbl-dependent EphA2 protein degradation is induced by ligand binding. Mol Cancer Res 1:79-87.

Wang B., Mysliwiec T., Krainc D., Jensen R.A., Sonoda G., Testa J.R., Golemis E.A. \& Kruh G.D. (1996) Identification of ArgBP1, an Arg protein tyrosine kinase binding protein that is the human homologue of a CNS-specific Xenopus gene. Oncogene 12:1921-1929.

Wang H.U. \& Anderson D.J. (1997) Eph family transmembrane ligands can mediate repulsive guidance of trunk neural crest migration and motor axon outgrowth. Neuron 18:383-396.

Wang H.U., Chen Z.F. \& Anderson D.J. (1998) Molecular distinction and angiogenic interaction between embryonic arteries and veins revealed by ephrin-B2 and its receptor Eph-B4. Cell 93:741-753.

Wang X., Roy P.J., Holland S.J., Zhang L.W., Culotti J.G. \& Pawson T. (1999) Multiple ephrins control cell organization in C. elegans using kinase-dependent and independent functions of the VAB-1 Eph receptor. Mol Cell 4:903-913.

Wang Z. \& Moran M.F. (1996) Requirement for the adapter protein GRB2 in EGF receptor endocytosis. Science 272:1935-1939.

Weinl C., Drescher U., Lang S., Bonhoeffer F. \& Loschinger J. (2003) On the turning of Xenopus retinal axons induced by ephrin-A5. Development 130:1635-1643.

Wennstrom S., Hawkins P., Cooke F., Hara K., Yonezawa K., Kasuga M., Jackson T., Claesson-Welsh L. \& Stephens L. (1994) Activation of phosphoinositide 3-kinase is required for PDGF-stimulated membrane ruffling. Curr Biol 4:385-393.

Wilkinson D.G. (2000) Eph receptors and ephrins: regulators of guidance and assembly. Int Rev Cytol 196:177-244.

Wilkinson D.G. (2001) Multiple roles of EPH receptors and ephrins in neural development. Nat Rev Neurosci 2:155-164.

Williams S.K., Spence H.J., Rodgers R.R., Ozanne B.W., Fitzgerald U. \& Barnett S.C. (2005) Role of Mayven, a kelch-related protein in oligodendrocyte process formation. J Neurosci Res 81:622-631.

Wilson S.W. \& Easter S.S., Jr. (1991) A pioneering growth cone in the embryonic zebrafish brain. Proc Natl Acad Sci U S A 88:2293-2296.

Wimmer-Kleikamp S.H., Janes P.W., Squire A., Bastiaens P.I. \& Lackmann M. (2004) Recruitment of Eph receptors into signaling clusters does not require ephrin contact. J Cell Biol 164:661-666.

Winslow J.W., Moran P., Valverde J., Shih A., Yuan J.Q., Wong S.C., Tsai S.P., Goddard A., Henzel W.J., Hefti F. \& et al. (1995) Cloning of AL-1, a ligand for an Eph-related tyrosine kinase receptor involved in axon bundle formation. Neuron 14:973-981. 
Wong L. \& Johnson G.R. (1996) Epidermal growth factor induces coupling of proteintyrosine phosphatase 1D to GRB2 via the COOH-terminal SH3 domain of GRB2. J Biol Chem 271:20981-20984.

Wunderlich L., Farago A. \& Buday L. (1999a) Characterization of interactions of Nck with Sos and dynamin. Cell Signal 11:25-29.

Wunderlich L., Farago A., Downward J. \& Buday L. (1999b) Association of Nck with tyrosine-phosphorylated SLP-76 in activated T lymphocytes. Eur J Immunol 29:1068-1075.

Wybenga-Groot L.E., Baskin B., Ong S.H., Tong J., Pawson T. \& Sicheri F. (2001) Structural basis for autoinhibition of the Ephb2 receptor tyrosine kinase by the unphosphorylated juxtamembrane region. Cell 106:745-757.

Xi R., Doan C., Liu D. \& Xie T. (2005) Pelota controls self-renewal of germline stem cells by repressing a Bam-independent differentiation pathway. Development 132:5365-5374.

Xu Q., Alldus G., Holder N. \& Wilkinson D.G. (1995) Expression of truncated Sek-1 receptor tyrosine kinase disrupts the segmental restriction of gene expression in the Xenopus and zebrafish hindbrain. Development 121:4005-4016.

Xu Q., Mellitzer G., Robinson V. \& Wilkinson D.G. (1999) In vivo cell sorting in complementary segmental domains mediated by Eph receptors and ephrins. Nature 399:267-271.

Xu Z., Fukuda T., Li Y., Zha X., Qin J. \& Wu C. (2005) Molecular dissection of PINCH1 reveals a mechanism of coupling and uncoupling of cell shape modulation and survival. J Biol Chem 280:27631-27637.

Yates P.A., Roskies A.L., McLaughlin T. \& O'Leary D.D. (2001) Topographic-specific axon branching controlled by ephrin-As is the critical event in retinotectal map development. J Neurosci 21:8548-8563.

Yin Y., Yamashita Y., Noda H., Okafuji T., Go M.J. \& Tanaka H. (2004) EphA receptor tyrosine kinases interact with co-expressed ephrin-A ligands in cis. Neurosci Res 48:285-296.

Yu H.H., Zisch A.H., Dodelet V.C. \& Pasquale E.B. (2001) Multiple signaling interactions of $\mathrm{Abl}$ and $\mathrm{Arg}$ kinases with the EphB2 receptor. Oncogene 20:39954006.

Yue Y., Chen Z.Y., Gale N.W., Blair-Flynn J., Hu T.J., Yue X., Cooper M., Crockett D.P., Yancopoulos G.D., Tessarollo L. \& Zhou R. (2002) Mistargeting hippocampal axons by expression of a truncated Eph receptor. Proc Natl Acad Sci U S A 99:10777-10782.

Zalevsky J., Lempert L., Kranitz H. \& Mullins R.D. (2001) Different WASP family proteins stimulate different Arp2/3 complex-dependent actin-nucleating activities. Curr Biol 11:1903-1913.

Zarich N., Oliva J.L., Jorge R., Santos E. \& Rojas J.M. (2000) The isoform-specific stretch of hSos1 defines a new Grb2-binding domain. Oncogene 19:5872-5883.

Zelinski D.P., Zantek N.D., Stewart J.C., Irizarry A.R. \& Kinch M.S. (2001) EphA2 overexpression causes tumorigenesis of mammary epithelial cells. Cancer Res 61:2301-2306. 
Zhang J. \& Hughes S. (2006) Role of the ephrin and Eph receptor tyrosine kinase families in angiogenesis and development of the cardiovascular system. J Pathol 208:453-461.

Zhang J.H., Cerretti D.P., Yu T., Flanagan J.G. \& Zhou R. (1996) Detection of ligands in regions anatomically connected to neurons expressing the Eph receptor Bsk: potential roles in neuron-target interaction. J Neurosci 16:7182-7192.

Zhao Z.S., Manser E. \& Lim L. (2000a) Interaction between PAK and nck: a template for Nck targets and role of PAK autophosphorylation. Mol Cell Biol 20:3906-3917.

Zhao Z.S., Manser E. \& Lim L. (2000b) Interaction between PAK and nck: a template for Nck targets and role of PAK autophosphorylation. Molecular \& Cellular Biology 20:3906-3917.

Zhou C., Cunningham L., Marcus A.I., Li Y. \& Kahn R.A. (2006) Arl2 and Arl3 regulate different microtubule-dependent processes. Mol Biol Cell 17:2476-2487.

Zhou R. (1997) Regulation of topographic projection by the Eph family receptor Bsk (EphA5) and its ligands. Cell Tissue Res 290:251-259.

Zhou R. (1998) The Eph family receptors and ligands. Pharmacol Ther 77:151-181.

Zhou X., Suh J., Cerretti D.P., Zhou R. \& DiCicco-Bloom E. (2001) Ephrins stimulate neurite outgrowth during early cortical neurogenesis. J Neurosci Res 66:10541063.

Zimmer G., Kastner B., Weth F. \& Bolz J. (2007) Multiple effects of ephrin-A5 on cortical neurons are mediated by SRC family kinases. J Neurosci 27:5643-5653.

Zisch A.H., Kalo M.S., Chong L.D. \& Pasquale E.B. (1998) Complex formation between EphB2 and Src requires phosphorylation of tyrosine 611 in the EphB2 juxtamembrane region. Oncogene 16:2657-2670.

Zisch A.H., Pazzagli C., Freeman A.L., Schneller M., Hadman M., Smith J.W., Ruoslahti E. \& Pasquale E.B. (2000) Replacing two conserved tyrosines of the EphB2 receptor with glutamic acid prevents binding of $\mathrm{SH} 2$ domains without abrogating kinase activity and biological responses. Oncogene 19:177-187.

Zou J.X., Liu Y., Pasquale E.B. \& Ruoslahti E. (2002) Activated SRC oncogene phosphorylates R-ras and suppresses integrin activity. J Biol Chem 277:18241827.

Zou J.X., Wang B., Kalo M.S., Zisch A.H., Pasquale E.B. \& Ruoslahti E. (1999) An Eph receptor regulates integrin activity through R-Ras. Proc Natl Acad Sci U S A 96:13813-13818.

Zukerberg L.R., Patrick G.N., Nikolic M., Humbert S., Wu C.L., Lanier L.M., Gertler F.B., Vidal M., Van Etten R.A. \& Tsai L.H. (2000) Cables links Cdk5 and c-Abl and facilitates $\mathrm{Cdk} 5$ tyrosine phosphorylation, kinase upregulation, and neurite outgrowth. Neuron 26:633-646.

Zvelebil M.J., Panayotou G., Linacre J. \& Waterfield M.D. (1995) Prediction and analysis of SH2 domain-phosphopeptide interactions. Protein Eng 8:527-533.

Zwick E., Bange J. \& Ullrich A. (2001) Receptor tyrosine kinase signalling as a target for cancer intervention strategies. Endocr Relat Cancer 8:161-173.

Zwick E., Bange J. \& Ullrich A. (2002) Receptor tyrosine kinases as targets for anticancer drugs. Trends Mol Med 8:17-23. 


\section{Curriculum vitae}

Tian-jing $\mathrm{Hu}$

\section{EDUCATION:}

- Rutgers University, New Brunswick, NJ

$\mathrm{Ph}$. D in Neurobiology, October 2007

- Nankai University, School of Life sciences, Tianjin, China B.S in Biochemistry and Molecular Biology, June 1999

\section{PUBLICATIONS:}

- Mistargeting Hippocampal Axons by Expression of a Truncated Eph Receptor

Yue Y, Chen ZY, Gale NW, Blair-Flynn J, Hu TJ, Yue X, Cooper M, Crockett DP, Yancopoculos GD, Tessarollo L, Zhou R PNAS August 6, 2002 vol 99, p10777-10782

- Regulation of Process Retraction and Cell Migration by EphA3 is Mediated by the Adaptor Protein Nck1 Hu T, Shi G, Mayer B, Zhou R In preparation 